In presenting the dissertation as a partial fulfiliment of the requirements for an advanced degree from the Georgia Institute of Technology, I agree that the Library of the Institution shall make it available for inspection and circulation in accordance with its regulations governing materials of this type. I agree that permission to copy from, or to publish from, this dissertation may be granted by the professor under whose direction it was written, or, in his absence, by the Dean of the Graduate Division when such copying or publication is solely for scholarly purposes and does not involve potential. financial gain. It is understood that any copying from, or publication. of, this dissertation which involves potential financial gain will not be allowed without written permission. 
An account of the research described herein was issued as a report by Oak Ridge National Laboratory.

Special permission was obtained from Mrs. J. Henley Crosland for placing the page numbers of this thesis at the center of the page instead of in line with the right hand margin, as recommended by the thesis instructions issued by the Craduate Division. This modification in the format permits the report to be printed on both sides of the paper without altering the pagination of the manuscript. 


\title{
AN INVESTIGATION \\ OF THE RESONANT PROPERTIES \\ OF SHORT EXPONENTIALLY AND \\ LINEARLY TAPERED LINES
}

\begin{abstract}
A THESIS
Presented to

the Faculty of the Graduate Division

Georgia Institute of Technology
\end{abstract}

In Partial Fulfillment

of the Requirements for the Degree

Doctor of Philosophy in the School

of Electrical Engineering

by

Merle Richard Donaldson

January 1957 
AN INVESTIGATION

OF THE RESONANT PROPERTIES

OF SHORT EXPONENTIALLY AND

LINEARLY TAPERED LINES

Approved:
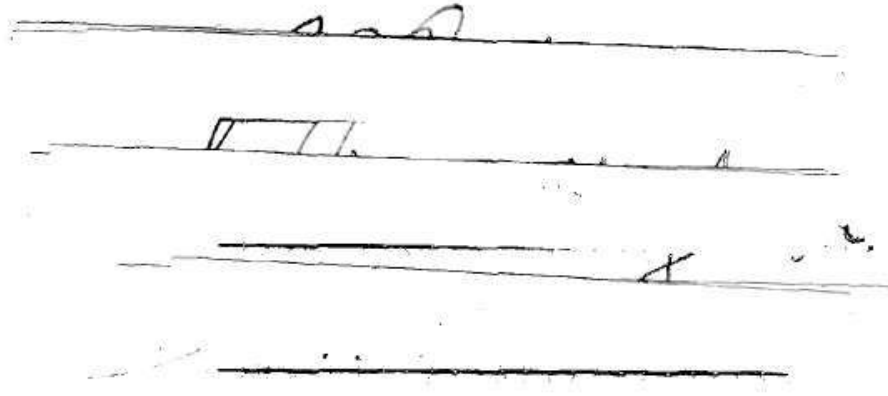

.

Date Approved by Chairman: 


\section{ACKNOWLEDGEMENT}

The cooperation of the Oak Ridge National Laboratory and the Georgia Institute of Technology in making possible the investigation herein reported is gratefully acknowledged by the author.

The author acknowledges great indebtedness to Professor B. J. Dasher and Dr. R. S. Livingston for their continued support, advice, and encouragement in this work.

The writer also wishes to thank Professor F. O. Nottingham, Professor M. L. Meeks, and Dr. A. Zucker for their interest in this work and for their helpful suggestions in preparing the manuscript.

Thanks are also due Mrs. G. Rudolph and Miss A. L. Smith for their untiring efforts in preparing the manuscript and to the ORNL Graphic Arts section for the preparation of the illustrations. Finally, the author wishes to express appreciation to his wife for her patience, encouragement, and assistance without which this work would not have been done. 
TABLE OF CONTENTS

$\begin{array}{lr} & \text { Page } \\ \text { Acknowledgement } & \text { ii } \\ \text { List of Tables } & \text { iv } \\ \text { List of Illustrations } & \text { V } \\ \text { List of Symbols } & \text { viii } \\ \text { Summary } & \text { xiv } \\ & \\ \text { Chapter } & \\ \text { I. Introduction } & 1 \\ \text { II. Review of the Basic Theory of the Uniform Line } & 13 \\ \text { IIJ. The Exponential Line } & 21 \\ \text { IV. The Linear Line } & 47 \\ \text { V. Experimental Results } & 75 \\ \text { VI. Discussion of Results } & 96 \\ \text { VII. Conclusions } & 122 \\ \text { VIII. Applications } & 130 \\ \text { IX. Recommendations } & 143\end{array}$

Appendix

I.

149

II.

150

III.

152

Bibliography 
-iv-

\section{LIST OF TABLES}

Table Page

1. Comparison of Measured and Computed Quarter- 93 Wave Frequencies 


\section{LIST OF ILLUSTRATIONS}

Figure

Page

1. Schematic Diagram of Dee System 4

2. Block Diagram of a Cyclotron R-F System Employing

(a) a Self-Excited Oscillator Circuit and

(b) a Master Oscillator-Power Amplifier Circuit

3. Oak Ridge National Laboratory (ORNL) 44-Inch Cyclotron Dee System

4. An Artist's Sketch of the ORNI 44-Inch Cyclotron 8

5. Si mplified Equivalent Circuit of Dee System Shown 9 in Figure 3

6. The General Transmission Line

14

7. (a) The Positively Tapered and

(b) the Negatively Tapered Exponential Line

8. A Sketch of the Input Impedance vs Length for the Open Line

9. A Sketch of the Input Impedance vs Length for the Shorted Line

10. Normalized Input Impedance vs Normalized Frequency of the Shorted Exponential Line

11. Normalized Input Impedance vs Frequency of the Shorted Exponential Line

12. Quarter-Wave Resonance vs Taper of the Lossless Exponential Line

13. Power Loss Ratio vs Taper of the Quarter-Wave Exponential Line

14. Quarter-Wave Resonance vs Taper of the Lossless Linear Line

15. Half-Wave Resonance vs Taper of the Open Lossless Linear Line

16. Half-Wave Resonance vs Taper of the Shorted Lossless Linear Line

17. A Plot of the Normalized Input Impedance vs Normalized Length for the Shorted Linear Line

66

18. Input Impedance vs Frequency for Two Linear and Two Uniform Lossless Lines 
19. Normalized Voltage and Current Standing Waves

on the Shorted Linear Line

20. Normalized Voltage and Current Standing Waves on the Shorted Linear Line

21. Power Loss Ratio vs Taper of the Quarter-Wave Linear Line

22. Experimental Uniform Strip Line

23. Method of Support for the Experimental Uniform

24. Experimental Linear Strip Line

25. Experimental Exponential Open Wire Line

26. Input Impedance vs Frequency of the Uniform Strip Lines

27. Deviation vs Frequency for the Four Uniform Strip Lines

28. Input Impedance vs Frequency for the Four Linear Strip Lines

29. Deviation vs Frequency for the Four Linear Strip Lines

30. Deviation vs Frequency for a Linear and a Uniform Strip Line

31. Input Impedance vs Frequency for a Linear and a Uniform Open Wire Line

32. Deviation vs Frequency for a Linear and a Uniform Open Wire Line

33. Basis for Comparing the

(a) Uniform, (b) Linear, and (c) Exponential.

Lines: $\mathrm{V}_{\mathrm{S}}=\mathrm{V}_{\mathrm{LS}}=\mathrm{V}_{\mathrm{ES}} ; \mathrm{Z}_{\mathrm{O}}=\mathrm{Z}_{1}=\mathrm{Z}_{2}$

34. Composite Relative Voltage and Current Distribution Exponential Lines

35. Relative Current Distribution vs Normalized Length for the Linear Line

36. Relative Voltage and Current Distribution vs Normalized Length for the Linear Line

37. Relative Voltage and Current Distribution vs Normalized Length for the Exponential Line 
38. Composite Curves of Normalized Input Impedance vs Normalized Length for Lossless Linear and Exponential Lines

39. Composite Curves of Normalized Input Impedance vs Length for Lossless Linear and Exponential Lines

40. Quarter-Wave Resonance vs Taper of the Lossless Linear and Exponential Lines

41. Power Loss Ratio vs Taper of the Quarter-Wave Linear and Exponential Lines

42. Normalized Input Impedance vs Normalized Frequency of Lossless Linear and Expoñential Lines

43. Normalized Input Impedance vs Frequency of Lossless Linear and Exponential Lines

44. Propagation of a Cylindrical Wave between SemiInfinite Conducting Planes

45. An Incremental Length of Tapered Line

46. Strip Line

47. Tuning Characteristics of Linear Lines vs Uniform Lines where the Length is Fixed and the Nominal Characteristic Impedance is the Tuning Variable

48. Tuning (6.7 to $11.8 \mathrm{Mc} / \mathrm{sec})$ by Varying the Length of a Uniform Line

49. Tuning (6.7 to $11.8 \mathrm{Mc} / \mathrm{sec})$ by Varying the Capacitance at the Open End of a Uniform Line

50. Tuning ( 6.7 to $11.8 \mathrm{Mc} / \mathrm{sec}$ ) by Pivoting a Linear Strip Line about the Open End so that $\mathrm{Z}_{0 \mathrm{~S}} / \mathrm{Z}_{\text {oo }}$

51. Power Amplifier Tank Circuit which Tunes from 100 to $300 \mathrm{Mc} / \mathrm{sec}$

52. (a) A Parallel Strip Line, (b) a Double Parallel Strip Line, and (c) a Shielded Coaxial Line Approximately Equivalent to a Strip Line 


\section{LIST OF SYMBOLS}

\section{Symbol}

$\mathrm{a}_{\mathrm{m}}$

$\mathrm{A}_{\mathrm{m}}$

$a_{n}$

$A_{n}$

A ok

$\mathrm{b}_{\mathrm{n}}$

$B_{n}$

$\mathrm{B}_{\mathrm{O}}$

B ok

C

$C_{1}, C_{2}, C_{3}, C_{4}$

C

c

$d, d_{m}, d_{o}, d_{s}$

D

E

$E_{f}$

$E_{\ell}$

$\mathrm{E}_{\mathrm{Z}}$

\section{Definition}

Odd-multiple $\lambda / 4$ resonant lengths of the open exponential line.

Odd-multiple $\lambda / 4$ resonant lengths of the shorted exponential line.

Half-wave resonant lengths of the open linear line.

Half-wave resonant lengths of the shorted linear line.

$\left[Y_{o k}+\left(z_{o} / \beta_{o} Z_{R}\right) Y_{1 k}\right]$, a constant.

Half -wave resonant leng ths of the open exponential line.

Half-wave resonant lengths of the shorted exponential line.

Shunt susceptance per unit length at $\underline{x}=0$.

$\left[J_{o k}+\left(z_{o} / \beta_{o} Z_{R}\right) J_{1 k}\right]$, a constant

Capacitance per unit length; a capacitance.

Constants of integration.

Capacitance per unit length at $\underline{x}=0$.

Speed of light.

Spacing between conductors of a strip line.

$\delta \mathrm{A}_{1}$.

Subscript denoting exponential line.

Frequency error, $\left(f_{0}-f_{o m}\right) / f_{0}$.

Length error.

Impedance error. 


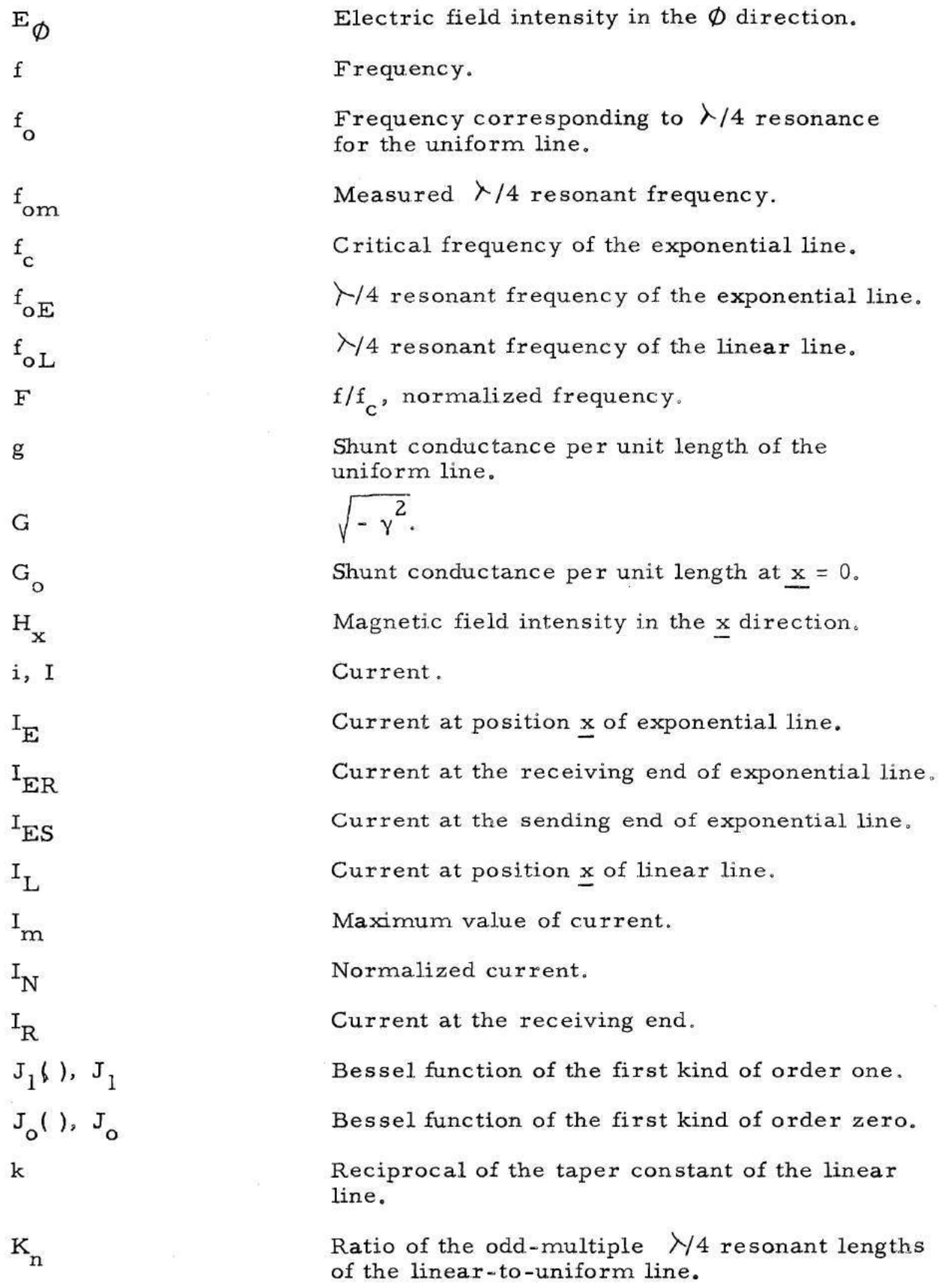




\begin{tabular}{|c|c|}
\hline $\bar{\ell}$ & $\left(r_{2}-r_{1}\right)$ \\
\hline$l$ & Length of line. \\
\hline$\ell_{\mathrm{n}}$ & $\begin{array}{l}\text { Odd-multiple } \lambda / 4 \text { resonant lengths of the } \\
\text { linear line. }\end{array}$ \\
\hline $\mathrm{L}$ & $\begin{array}{l}\text { Inductance per unit length; subscript denoting } \\
\text { the linear line. }\end{array}$ \\
\hline$\ell_{0}$ & $\begin{array}{l}\text { Length of uniform line corresponding to } \lambda / 4 \\
\text { resonance. }\end{array}$ \\
\hline$L_{0}$ & Inductance per unit length at $\underline{x}=.0$ 。 \\
\hline bon & $\begin{array}{l}\text { Odd-multiple } \lambda / 4 \text { resonant lengths of the } \\
\text { uniform line. }\end{array}$ \\
\hline $\mathrm{p}$ & Power loss for an incremental length, $\mathrm{dx}$. \\
\hline $\mathrm{P}$ & Power loss. \\
\hline$P_{\circ}$ & Power loss in a uniform $\lambda / 4$ line. \\
\hline$P_{O E}$ & Power loss in an exponential $\lambda / 4$ resonator. \\
\hline$P_{O L}$ & Power loss in a linear $\lambda / 4$ resonator. \\
\hline $\mathrm{r}$ & $\begin{array}{l}\text { Series resistance per unit length of the uniform } \\
\text { line; distance variable. }\end{array}$ \\
\hline $\mathrm{R}$ & $\begin{array}{l}\text { Resistance; subscript denoting the receiving end } \\
\text { values. }\end{array}$ \\
\hline$R_{0}$ & Series resistance per unit length at $\underline{x}=0$. \\
\hline$R_{p}$ & Parallel resonant resistance. \\
\hline$R_{\text {po }}$ & $\begin{array}{l}\text { Parallel resonant resistance of the uniform } \\
\text { line. }\end{array}$ \\
\hline $\mathbf{S}$ & Subscript denoting sending end values. \\
\hline $\mathbf{s}$ & Arc length. \\
\hline$t$ & Time variable. \\
\hline $\mathrm{u}$ & Distance variable. \\
\hline$\overline{\mathrm{u}}$ & $\mathrm{k} \mp \ell$. \\
\hline $\mathrm{v}, \mathrm{V}$ & Voltage. \\
\hline
\end{tabular}




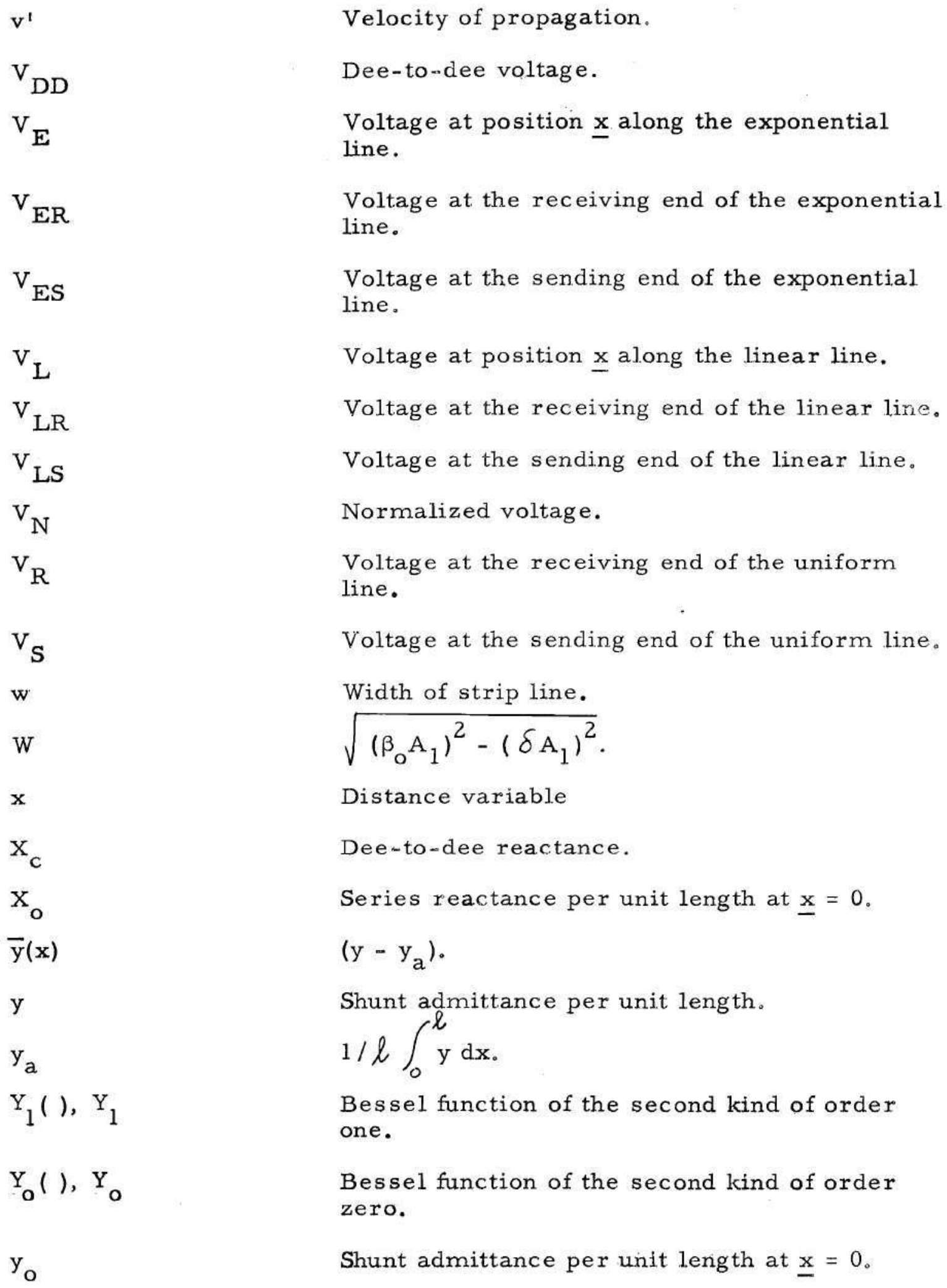


$z_{0}$

Z

$\mathrm{z}_{\mathrm{a}}$

$\mathrm{Z}$ a

$\bar{z}(x)$

$\mathrm{Z}_{\mathrm{E}}$

$\mathrm{Z}_{\text {L }}$

$\mathrm{Z}_{\mathrm{N}}$

Z

Z oo

$\mathrm{Z}_{\mathrm{OE}}$

$\mathrm{Z}_{\mathrm{OL}}$

$\mathrm{Z}_{\text {or }}$

$\mathrm{Z}_{\text {oS }}$

$\mathrm{z}_{\text {ou }}$

$\mathrm{Z}_{\text {ox }}$

$\mathrm{Z}_{\mathrm{R}}$

$\mathrm{z}_{\mathrm{S}}$

a

$\beta$

$\beta_{0}$

$\beta_{\mathrm{OE}}$
Series impedance per unit length.

Series impedance per unit length at $\underline{x}=0$.

Input impedance of the uniform line at position $\mathrm{x}$.

$1 / \ell \int_{0}^{l} z(x) d x$.

$\sqrt{z_{a} / y_{a}}$.

$\left(z-z_{a}\right)$ 。

Input impedance of exponential line at position $\underline{x}$.

Input impedance of linear line at position $\underline{x}_{0}$

Normalized input impedance.

Characteristic impedance of a uniform line.

Nominal characteristic impedance at $\underline{x}=0$.

Nominal characteristic impedance at position $\underline{x}$ of the exponential line.

Nominal characteristic impedance at position $\underline{x}$ of the linear line.

$377(\phi \mathrm{r} / \mathrm{w})$.

Nominal characteristic impedance at $\underline{x}=\ell$.

$377(d / w)$.

Nominal chracteristic impedance at $\underline{x}=\underline{x}$.

Impedance at receiving end.

Input impedance at $\underline{x}=\ell$.

Attenuation constant of the uniform line.

$$
\sqrt{\beta_{0}^{2}-d^{2}} \text {. }
$$

Phase constant of the uniform line, $\omega \sqrt{L_{0} C_{0}}$.

$\omega \sqrt{L_{0} C_{0}}$ for the exponential line. 


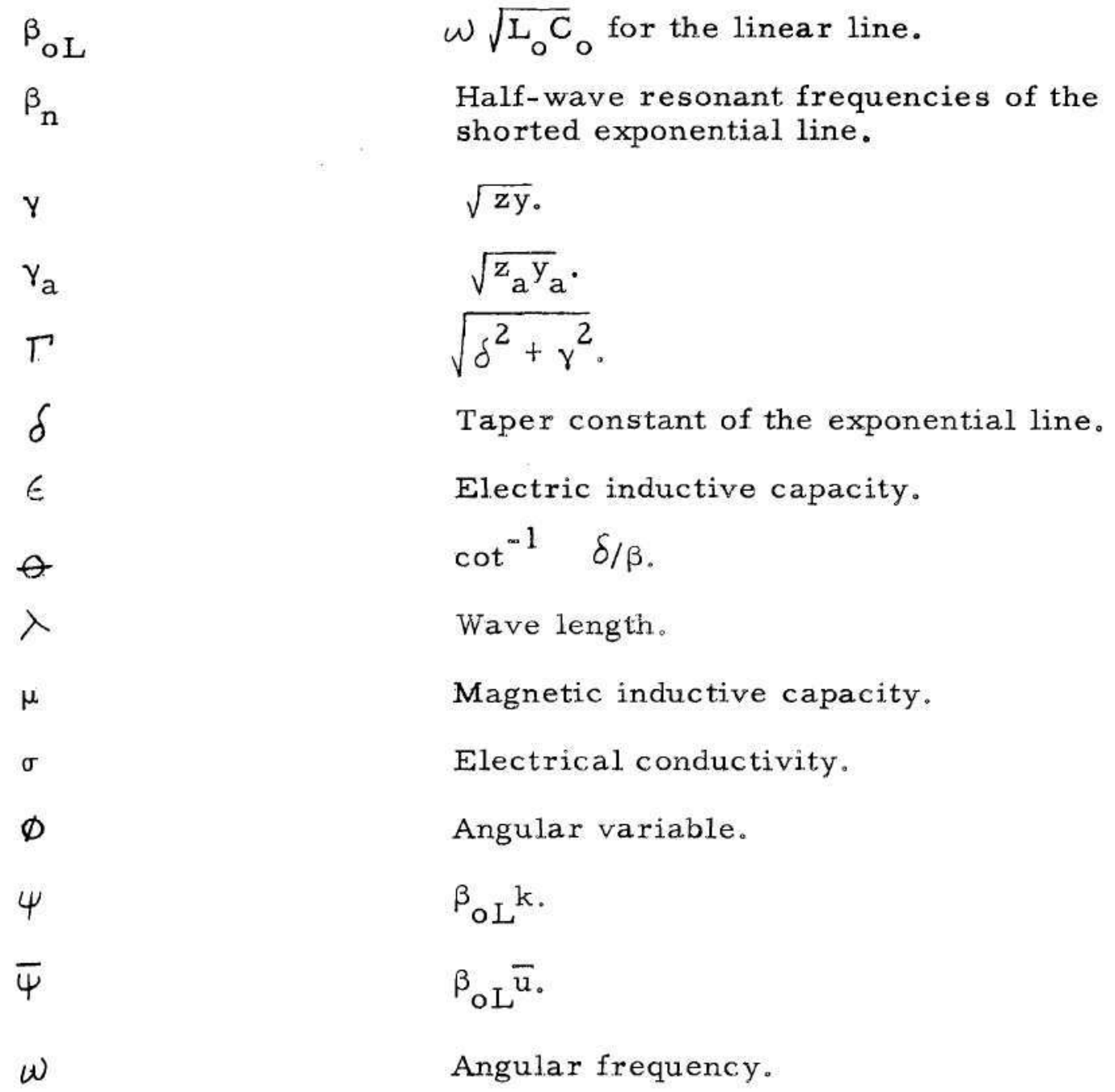


SUMM A R Y 


\section{SUMMARY}

The theory of the uniform line has been treated extensively and is well known. For many years, sections of uniform line have been used as high-Q resonant circuits for various purposes. One of the applications has been in the field of cyclotron engineering in which, traditionally, a quarter-wave uniform line has been used to excite the accelerating electrodes of the cyclotron to high $\mathrm{r}-\mathrm{f}$ voltages. A critical study of the requirements of this quarter-wave line leads to the following question: What are the advantages and disadvantages of using a nonuniform line for this purpose? This study was motivated by the above question and, for that reason, cyclotron problems are emphasized in the work. The exponentially and linearly tapered lines are chosen for study with emphasis on the linear line since it can be fabricated practically.

The object of the investigation is two-fold: first, to develop the theory of the linear line and to extend the theory of the resonant properties of the exponential line with emphasis on the power and tuning properties of both lines; second, to provide unified results comparing the resonant properties of the exponential, linear, and uniform lines.

The classical approach employing the method of ordinary transmission line theory is used to develop analytical expressions for both of the tapered lines. For simplicity, emphasis is placed upon lossless lines. The resulting voltage and current expressions for the expo nential line are, of course, in terms of exponential and circular functions. For the linear line, the expressions for voltage and current are in terms of Bessel functions of the first and second kinds of orders zero and one. Experimental data are presented to verify the mathematical results.

Various illustrations of current, voltage, power, and input impedance properties are presented for the two tapered lines. Composite curves are provided to show a concise comparison of the similarities 
and differences among the exponential, linear, and uniform lines. In addition, the tuning properties of the tapered lines are discussed.

It is shown that quarter-wave linear (and exponential) lines can be designed which will operate at a given frequency and which will be either longer, or shorter, than a uniform line operating at the same frequency. The extent to which the tapered lines are longer (or shorter) depends on the ratio of nominal characteristic impedances at the ends of the tapered lines only. Similarly, the results show that quarter wave linear (or exponential) lines can be designed which will resonato at frequencies above, or below, the resonant frequency determined by a uniform line of the same length.

It is shown that quarter-wave linear (or exponential) lines, whose nominal characteristic impedance decreases from the shorted end toward the open end, require less power to obtain a given voltage at the open end than the comparable uniform line (where both lines are operating at the same frequency and have the same nominal charac. teristic impedance at the open end). The ratio of power dissipated by the tapered line to that of the uniform line is a function of the ratio of nominal characteristic impedances at the ends of the tapered line only. Conversely, it is shown that quarter-wave linear (or exponential) lines, whose nominal characteristic impedance increases from the shorted end toward the open end, require more power than the comparable uniform line.

With respect to tuning, it is shown that linear (and exponential) lines add considerable design flexibility, and can be used advantageous $1 y$, in the solution of wide-range tuning problems in which the line is the variable parameter. The theory of the linear line is illustrated by two examples, one of which was the cyclotron problem that motivated this research. 


\section{CHAPTER I}

\section{INTRODUCTION}

Definition of the Problem. - - For many years, the electrical properties of transmission lines have been employed for useful purposes in electrical networks. Sections of line have been used for tank circuits, voltage transformers, circuit components, frequency stabilizers, impedancemmatching devices, and many other elements. In general, it is possible to catalog the different applications in many ways. For convenience in this writing, the applications will be classified according to the mode of operation. The two categories will be termed nonreso$\underline{\text { nant }}$ and resonant operation, respectively. Specifically, the former will denote the absence and the latter will imply the presence of standing waves on the section of line under consideration.

This investigation is a study of the resonant properties of the exponentially and the linearly tapered lines. Special emphasis is placed on the linear line and its application to one class of particle accelerators, the fixed-frequency cyclotron. *

A study of the nonresonant properties of these lines is not of interest in this work. History of the Problem. - - Historically, the inception of the research reported herein occurred in connection with the preliminary design of a variable-frequency cyclotron. ${ }^{* * * *}$ Since there are approximately only

* frequency cyclotron.

**A variable-frequenc y cyclotron is defined to mean a conventional cyclotron which is capable of operating at several discrete frequencies over a rather large range (for example, from 5 to $12 \mathrm{Mc} / \mathrm{sec}$ ). It is not to be confused with the f-m cyclotron, also known as the synchrocyclotron, which requires the frequency to vary continuously with time during the acceleration period of the particle. For an excellent survey article on all classes of accelerators, the reader is referred to the Bibliography. 1 
30 cyclotrons in the United States, the engineering details are not widely known. For this reason it seems desirable to review quite briefly the major components of such machines. In addition, a portion of one of the major components which is of direct interest to this work will be described in detail.

In the past two decades since the invention of the cyclotron, the electrical engineering problems of accelerators have not been given adequate attention from a mature engineering point of view. This situation has largely been due to three reasons. First, the financial resources available to the educational institutions which were building cyclotrons were never more than barely adequate. Second, the design groups, in many cases, consisted of individuals whose primary interest and training were in research in the physical sciences rather than in engineering. These factors limited the interest and motivation for mature development of engineering problems. Third, each machine was designed as a single installation and "tailored" to the budget, building, and personnel available; this discouraged the process of reducing the engineering art to an engineering science.

Currently there is a lively interest in extending the present limits of the cyclotron for a research tool as a result of the recent widespread impetus toward atomic energy and nuclear science. To paraphrase BUSINESS WEEK (April 10, 1954)... since World War II there has developed "a race for bigger and more powerful" accelerators to aid nuclear research. The scientists believe "that finding what the atom is made of ... will unlock the secrets of the universe." For this type of research, the cyclotron is one of the most widely-used machines in the family of accelerators. In addition, the cyclotron has an important function in producing isotopes for therapeutic purposes.

One recent trend in the cyclotron art is toward more versatile fixed-frequency cyclotrons that will operate at several discrete frequencies. ${ }^{2,3,4}$ This interest has been stimulated by the experimentalist's need to obtain adjustable variations in particle energy at the target, as well as by his need to accelerate several different particles with the same machine. These needs require the cyclotron to operate at sever al specific frequencies over a range of the order of two to one which 
complicates the conventional cyclotron design problems considerably. As a result many phases of the cyclotron art are quite fertile areas for careful and precise engineering work.

For the benefit of the readers who are not familiar with the conventional cyclotron, the following brief review of the major components is presented.

The cyclotron is a relatively large complex machine whose basic purpose is to accelerate ionized particles to relatively high energies to bombard a given target material, thereby creating nuclear reactions. Operating on the well-known principle of the interaction between a magnetic field and an ionized particle traveling perpendicular to this field, the machine accelerates particles to high energies by passing the ions repeatedly between two accelerating electrodes which are excited by a $r-f$ voltage. The cyclotron has four major components: a source of ionized particles which is similar to the filament in a vacuum tube: a vacuum chamber which provides for unrestricted movement of the ions, and within which the source and accelerating electrodes are enclosed; a magnetic field which constrains the particles to spiral paths so that they may be acted upon repeatedly by the accelerating voltage; and the accelerating electrodes which impart energy to the ions each time they pass through the accelerating gap. These four components are indicated schematically in Fig. 1 .

While the $r-f$ circuitry is incidental to the function of the accelerating electrodes, the apparatus used to excite these D-shaped electrodes to the high $r-f$ potential required is an important practical consideration. This apparatus is called the $r-f$ system and is composed of a d-c power supply, an $r-f$ self-excited oscillator, two $r-f$ transmission circuits, and the resonant dee system as shown in block diagram in Fig. 2a. (An alternative system is shown in Fig. 2b.) The last component is of particular interest to this study and is composed of three elements: the accelerating electrodes, called the dees; the shorted section of transmission line, called the dee stems and shorting bar; and the box or liner within which the dees, the dee stems, and the shorting bar are enclosed for $r-f$ shielding purposes. Fig. 3 is a photograph of the Oak Ridge National Laboratory 44-Inch Cyclotron dee 
$-4-$

UNCLASSIFIED
ORNL-LR-DWG 18025

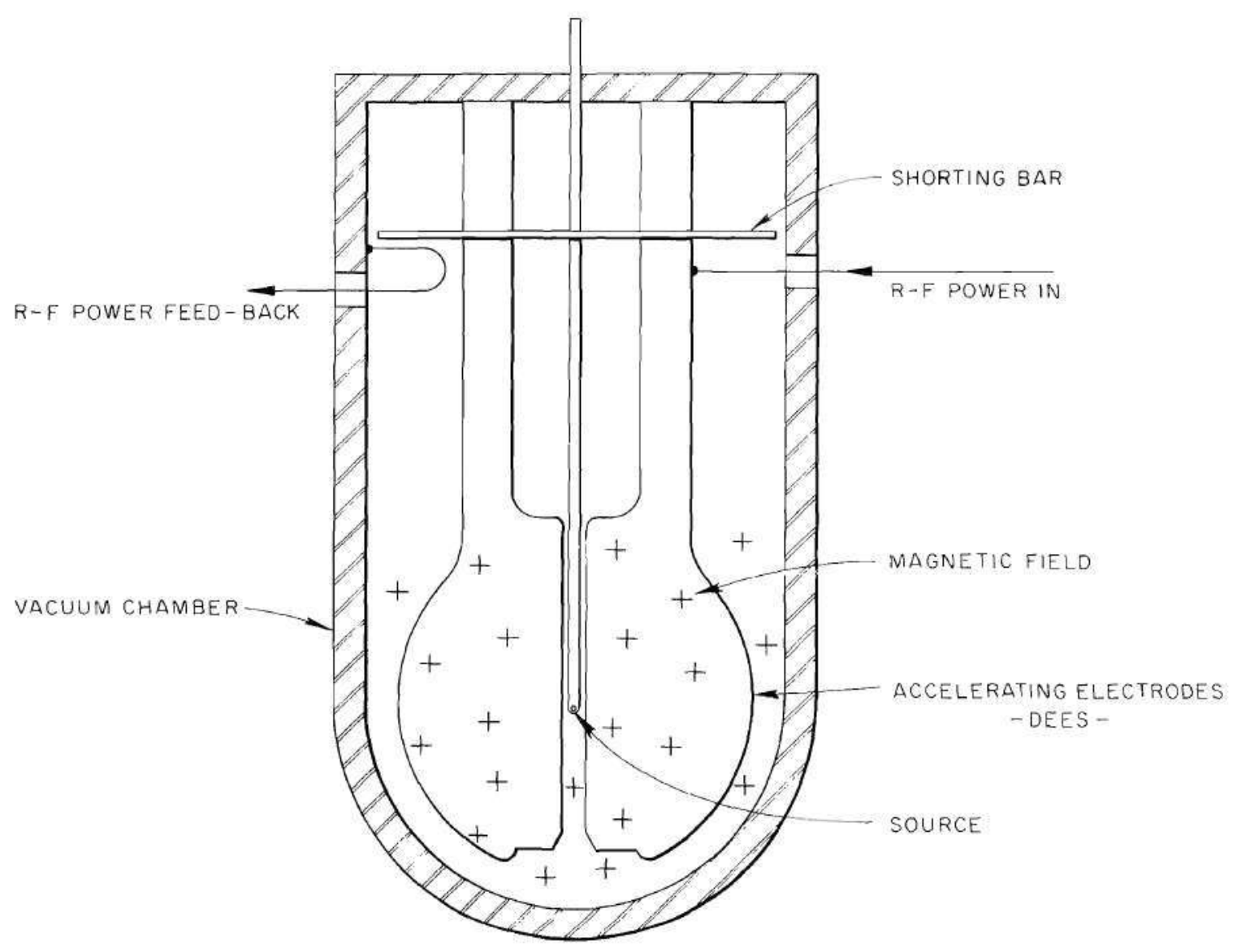

Fig. 1. Schematic Diagram of Dee System 
UNCLASSIFIED

ORNL-LR-DWG 18026

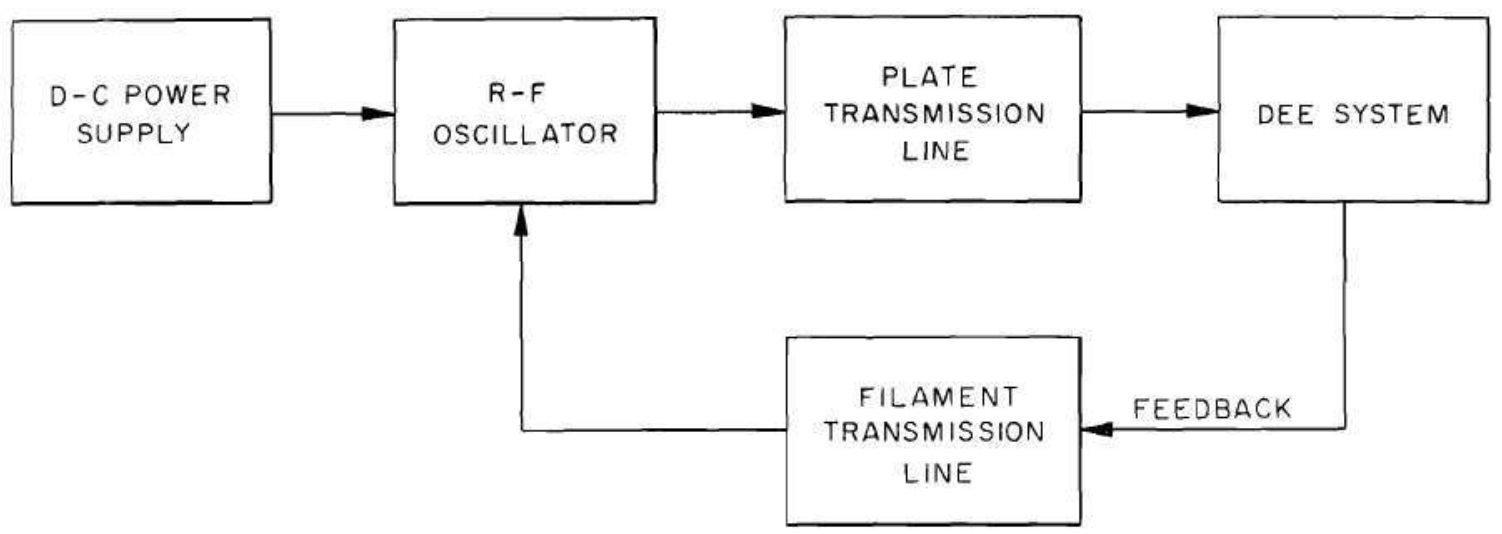

(a)

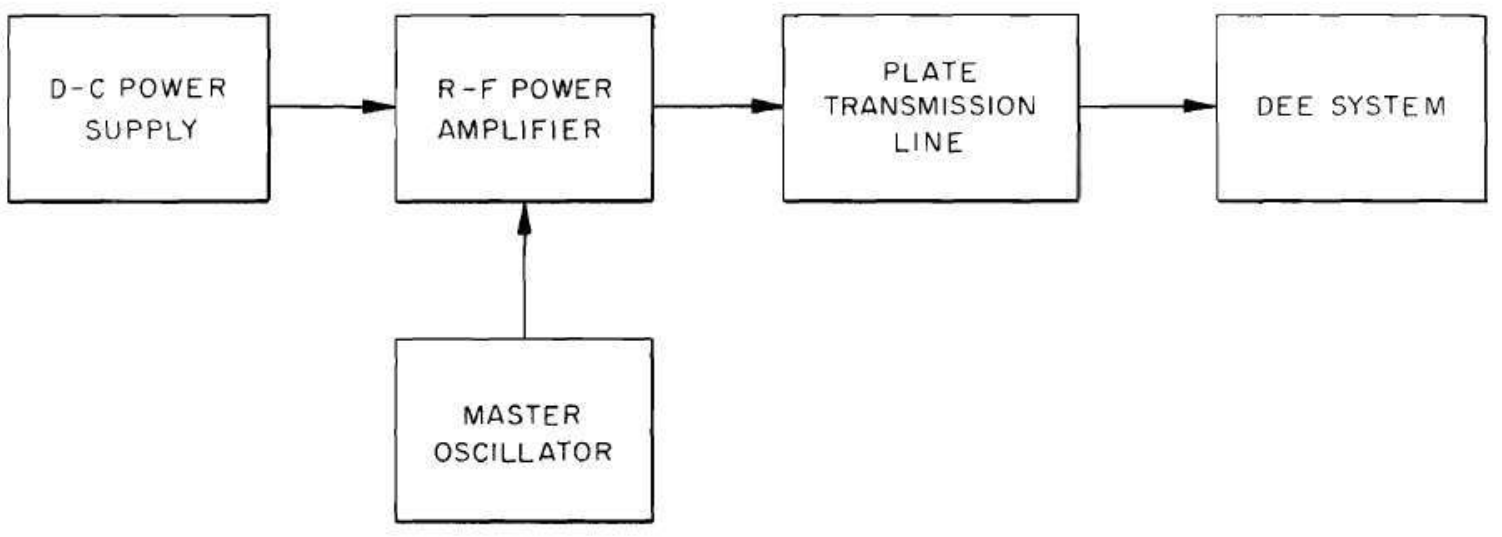

(b)

Fig. 2. Block Diogram of a Cyclotron R-F System Employing

(a) o Self-Excited Oscillator Circuit and

(b) a Master Oscillator-Power Amplifier Circuit 


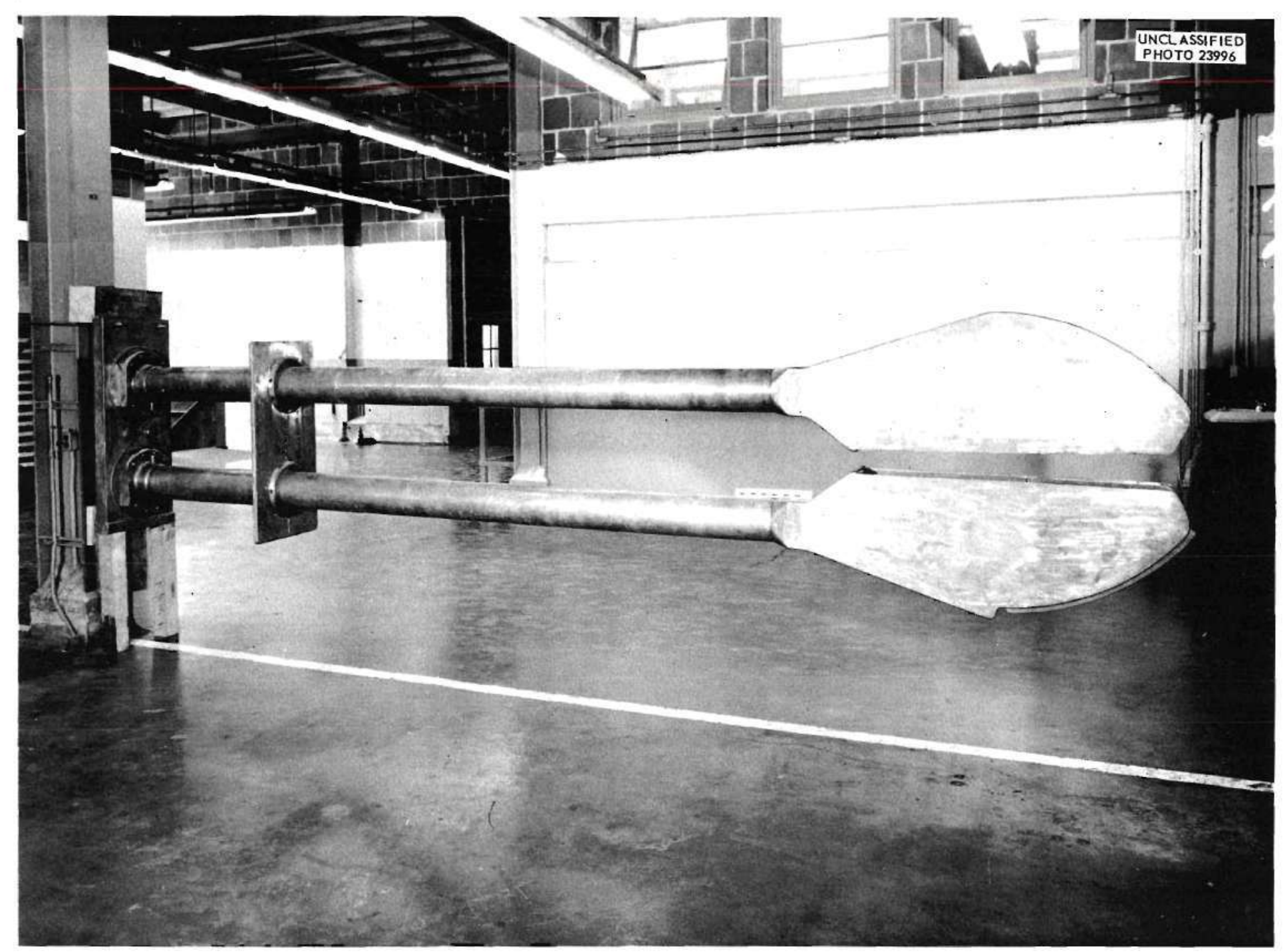

Fig. 3. Oak Ridge National Laboratory (ORNL) 44-Inch Cyclotron Dee System. 
system showing the dees, the dee stems, and the shorting bar, but not the $\mathrm{r}-\mathrm{f}$ enclosure. Fig. 4 is an artist's sketch of the same dee system installed showing its relationship to other components of the cyclotron.

With the above preliminary remarks, the dee system, which is the portion of interest, is discussed in detail for a better understanding of the problem that motivated the research. The simplified equivalent electrical circuit for the dee system shown in Fig. 3 is indicated in Fig. 5. Since the dees behave essentially as a large capacitance, C, and since the desired accelerating voltage, $V_{D^{\prime}}$, is quite high (ordinarily from 100 to $150 \mathrm{kv}$ for the ORNL 44-Inch Cyclotron), the capacitance must be tuned for resonance at the operating radio frequency, $f_{0}$. To accomplish this, the dee stems and the shorting bar must provide the necessary inductance. The conditions for this resonance are expressed as follows:

$$
1 / 2 \pi f_{0} C=Z_{0} \tan \left(2 \pi f_{0} / v^{\prime}\right) l
$$

where $Z_{0}$ is the characteristic impednace of the line and $\underline{y}^{\prime}$ is the velocity of propagation. Ordinarily for the fixed-frequency cyclotron, the frequency and the capacitance are given and $z_{0}$ and $l$ are chosen to satisfy (1).

Then in principle, if (1) is to be satisfied at several specific frequencies over a rather wide range, the capacitance, or the length, may be varied. However, it has been shown ${ }^{5}$ that disadvantages arise when either $C$ or $l$ is used as the tuning variable. This leaves $Z_{0}$ as the last possibility and raises the following question: What are the advantages and disadvantages of using a nonuniform line for the dee stems instead of the traditional uniform line? Thus the problem was born; and, although the motivation was a tuning problem, the pos. sibility that nonuniform lines might require less power dissipation was recognized also.

Purpose of the Research.--For the purpose of studying the cyclotron problem, the exponentially and linearly tapered lines were chosen for investigation with the objective of correlating their resonant properties 


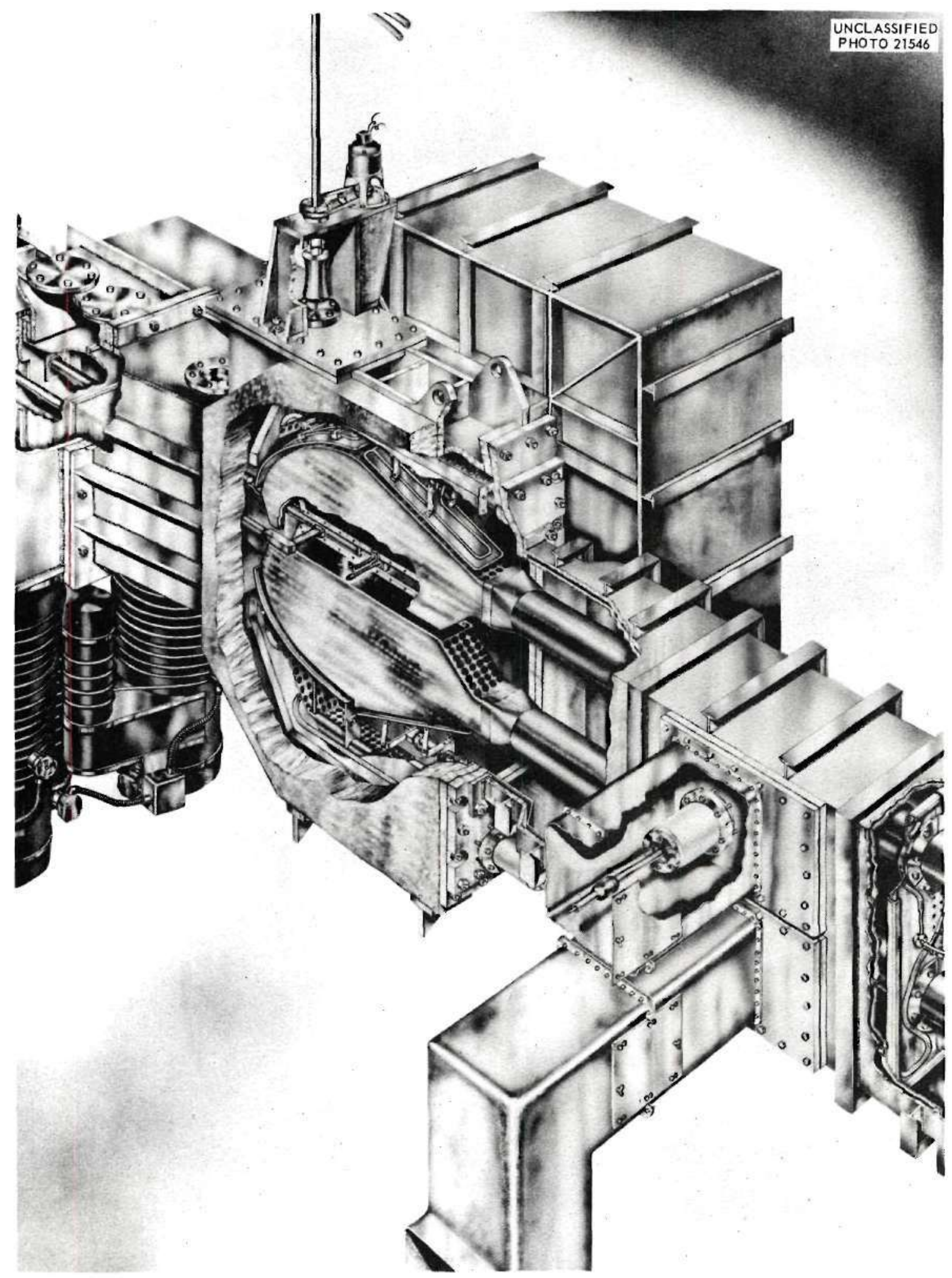

Fig. 4. An Artist's Sketch of the ORNL 44-Inch Cyclotron. 


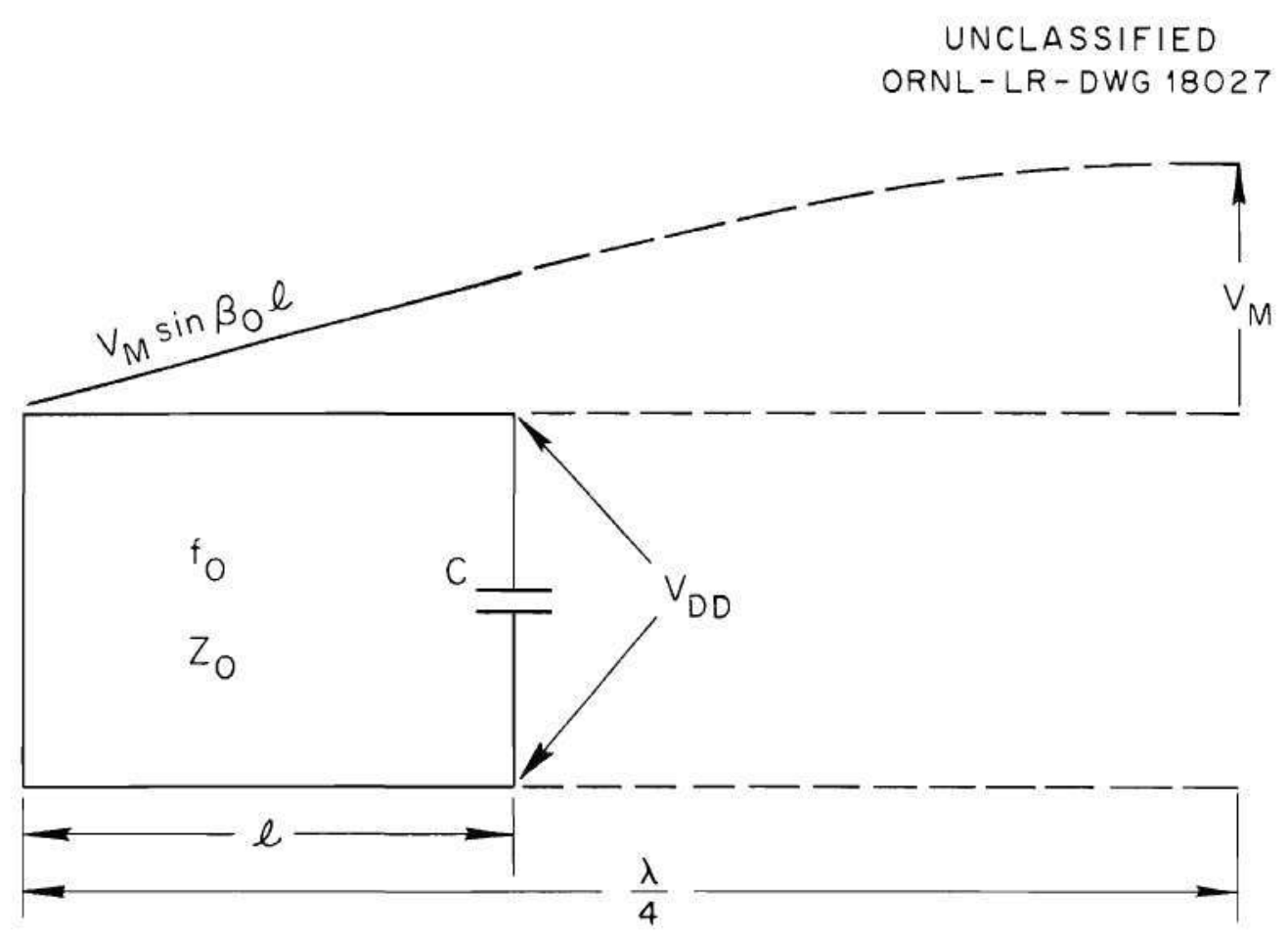

Fig. 5. Simplified Equivalent Circuit of Dee System Shown in Fig. 3. 
with the analogous properties of the uniform line.

Review of the Literature. - Although the literature on the general nonuniform line dates back to Heaviside ${ }^{6}$ (1882), the more important papers appeared during the early part of the twentieth century. Ravut ${ }^{7}(1920)$ obtained a general solution of the nonuniform line in the form of an infinite series requiring the assumption that the line constants were differentiable with respect to distance along the line. Subsequently, Carson ${ }^{8}$ (1921) obtained the solution of the general case in the form of an infinite series of integrals using the assumption that the line constants were integrable. This, of course, is a much less restrictive assumption, since discontinuities are permissible. Later, Thomas ${ }^{9}$ (1929) presented a treatment aimed at simplifying Ravut's method. Recently, Schelkunoff ${ }^{10}$ (1943) published a more comprehensive treatment similar to Carson's work.

Heaviside's" original study of the "Bessel Cable" treated the case in which the series inductance and shunt conductance were neglected, and the series resistance and shunt capacitance varied linearly with distance along the line. Heaviside obtained the solution in terms of Bessel Functions of zero order. Later, Ballantine 11 (1927) obtained a solution in terms of Bessel. Functions for the case where the series impedance and the shunt admittance varied as the same power of the distance from the origin. Subsequently, Arnold and Bechberger ${ }^{12}$ (1931) and, separately, Federici ${ }^{13}$ (1931) solved the case in which the series impedance varied linearly with distance and the shunt admittance was constant. Then Arnold and Taylor ${ }^{14}$ (1932) extended the treatment of this last case by allowing the series resist. ance and the series inductance to vary linearly with distance at dif ferent rates. Finally, Starr ${ }^{15}$ (1932) unified the treatments of Heaviside, Ballantine, Arnold and Bechberger, and Federici by obtaining a more general solution in terms of Bessel. Functions for the case in which the series impedance and the shunt admittance varied as any power of the distance along the line. The linear line studied

${ }^{* *}$ Heaviside, op. cit. , p. 172-175. 
in this investigation is included as a particular case of Starr's general solution. The series impedance varies linearly and the shunt admittance varies inversely with distance for this line.

Burrows ${ }^{16}$ (1938) developed the theory of the exponential line and was perhaps the first writer to hint at the resonant possibilities of tapered lines. In a more recent paper, Walker and Wax 17 (1946) derive a first-order differential equation for the voltage coefficient of a nonuniform line and show how to calculate the resonant wave lengths of linear lines. Selgin ${ }^{18}$ (1946) presented an extended treatment of the exponential line similar to Burrows but including a section on the properties of tuning stubs. This section on tuning stubs is pertinent to this investigation and is the only paper known to the author which discusses the resonant properties of nonuniform lines to any significant extent.

Other papers 19,20 on nonuniform lines have appeared, but none are pertinent to this investigation. The papers of Starr, and Walker and $W$ ax on the linear line, and those of Burrows and Selgin on the exponential line, are the four most directly related to this study. Procedure Used in Research. - - The method for conducting the investi gation was decided upon at the inception of the research. It was felt that the linear line held the most interest since it could be fabricated practically. However, since the exponential line was known to submit to solution (and the linear line was not known to do so), it was believed that a development of the exponential. line aimed at the solution of the cyclotron problem would greatly aid the study of the linear line. Hence, the procedure chosen was to attempt a mathematical development of both lines by using the exponential line as a guide, and to provide an experi. mental study of the areas for which a mathematical solution was impractical. A mathematical treatment using the methods of "ordinary transmission line theory" was employed, viz,

1. The energy is assumed to be transmitted down the transmission line in the transverse electromagnetic mode (TEM).

2. The general differential equations for voltage and current are formulated from lumped circuit methods by defining a 
series impedance and a shunt admittance per unit length of line.

3. The differential equations are solved, subject to the boundary conditions, for voltage and current as a function of position along the line.

4. Harmonic functions of time are assumed since the interest is in a-c steady state.

More rigorous analyses ${ }^{21,22}$ using Maxwell's equations and field theory techniques show that ordinary transmission line theory is quite adequate.

The procedure described above proved to be suitable and was followed.

Scope of the Investigation. --This study has two objectives: first, to develop and extend the theory of the resonant properties of the exponential and linear transmission lines with emphasis on the tuning and power problems of the cyclotron; second, to provide unified results correlating the resonant properties of these two tapered lines with analogous properties of the uniform line. The results are expected to be useful for general application, and in addition, to serve as a guide for future studies of the properties of other types of nonuniform line -- which may possibly lead to elements with even more novel and desirable resonant properties. 
CHAPTER II

\section{REVIEW OF THE BASIC THEORY \\ OF THE UNIFORM LINE}

Introduction. - This chapter presents the general nomenclature and symbolism to be used in the remaining portion of this paper. In addition, the mathematical expressions for the uniform line, which are pertinent to the following chapters, are listed for reference.

Nomenclature and Symbolism. -- Consider the general transmission line indicated in Fig. 6. The line has two basic parameters, namely, the shunt admittance, $\underline{y}$, and the series impedance, $\underline{z}$, per unit length of line. The square root of their ratio is called the nominal characteristic impedance. The manner in which this quantity $(\sqrt{\mathrm{z} / \mathrm{y}})$ is allowed to vary with position along the line defines the particular type of line to be considered. For example, if $\underline{z}$ and $\underline{y}$ are constant with respect to dis tance, the $\sqrt{\mathrm{z} / \mathrm{y}}$ is constant, and the uniform line is defined. As shown in Fig. 6, the distance is measured from the receiving end of the line. The voltage, current, and impedance at the sending end and the receiv ing end are denoted by the subscripts "S" and " $R$ ", respectively. Similar quantities at an arbitrary distance, $x$, from the origin are denoted by the absence of subscripts.

In general,

$$
\begin{aligned}
& v=v(x, t), \\
& i=i(x, t) .
\end{aligned}
$$

However, since this work is concerned with a-c steady state and not with transient analysis, the expressions for $\underline{v}$ and $\underline{i}$ may be written in the customary form, *

* See, for example, W. C. Johnson, Transmission Lines and Networks, New York: McGraw-Hill Book Company, Inc., 1950. Chap. 2. 


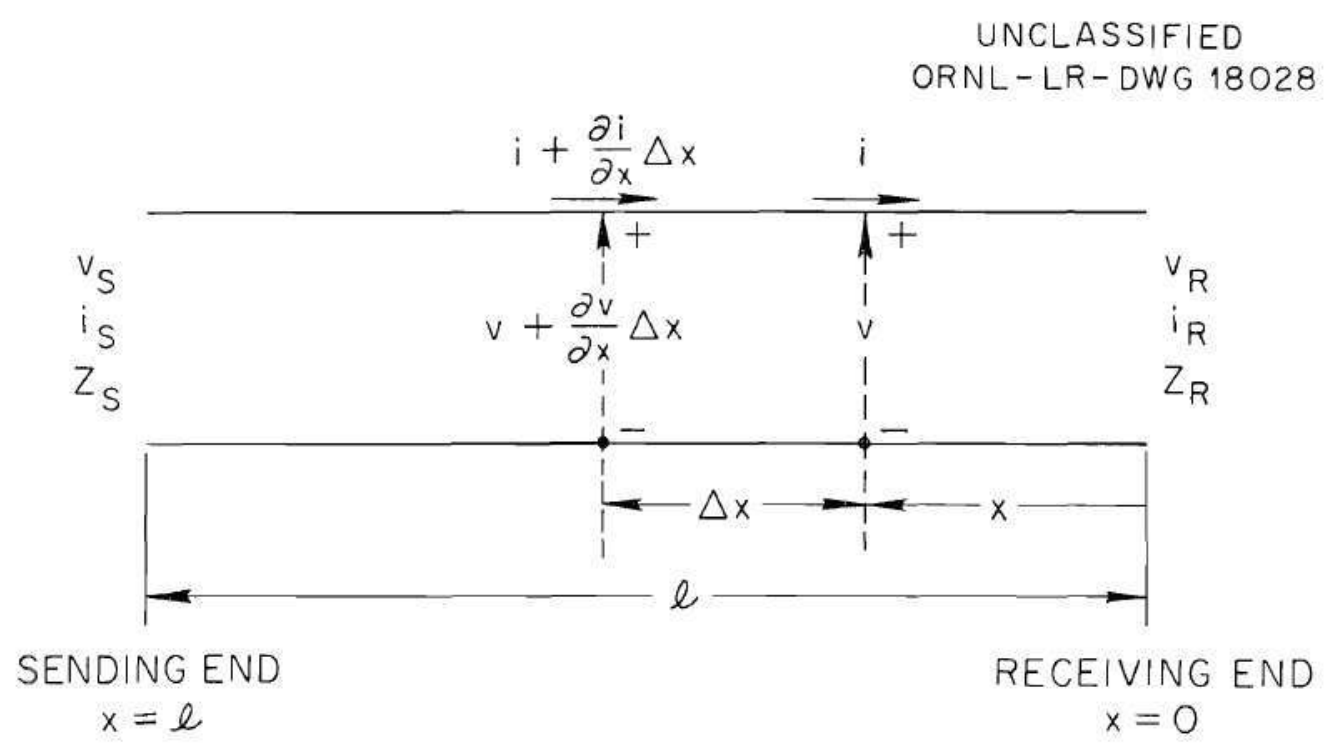

Fig. 6. The General Transmission Line. 


$$
\begin{aligned}
& v=V(x) e^{j \omega t} \equiv V e^{j \omega t}, \\
& i=I(x) e^{j \omega t} \equiv I e^{j \omega t},
\end{aligned}
$$

where it is understood that only the real part of $\mathrm{e}^{j \omega t}$ is used. With the above terminology, the partial differential equations for the general transmission line may be formulated from Fig. 6 as follows:

$$
\begin{aligned}
& {[v+(\partial v / \partial x) \Delta x]-v=i z \Delta x,} \\
& {[i+(\partial i / \partial x) \Delta x]-i=v y \Delta x,}
\end{aligned}
$$

from which

$$
\begin{aligned}
& \partial v / \partial x=i z, \\
& \partial i / \partial x=v y,
\end{aligned}
$$

By differentiating (8) and (9) with respect to $\underline{x}$, the following partial differential equations are obtained:

$$
\begin{aligned}
& \partial^{2} v / \partial x^{2}=i \partial z / \partial x+z \partial i / \partial x, \\
& \partial^{2} i / \partial x^{2}=v \partial y / \partial x+y \partial v / \partial x,
\end{aligned}
$$

When (4) and (5) are used in equations (10) and (11) the factor $e^{j \omega t}$ is common to all terms and may be cancelled. The remaining expressions are functions of $x$ only and the partials become total derivatives. When (8) and (9) are substituted in (10) and (11) the differential equations for the general line become

$$
\begin{aligned}
& d^{2} V / d x^{2}-(d z / z d x) d V / d x-z y V=0, \\
& d^{2} I / d x^{2}-(d y / y d x) d I / d x-z y I=0,
\end{aligned}
$$


The Uniform Line. As has been mentioned above, if $\underline{z}$ and $\underline{y}$ are constants, equations (12) and (13) become

$$
\begin{aligned}
& d^{2} V / d x^{2}-z y V=0, \\
& d^{2} I / d x^{2}-z y I=0,
\end{aligned}
$$

which are the well-known expressions for the uniform line. Since the solutions of these equations may be found in any standard text ${ }^{*}$ on transmission lines, they are not solved here. However, for reference purposes in the following chapters, the important expressions resulting from the solution of (14) and (15) are summarized and discussed below.

The general expressions for voltage and current are

$$
\begin{aligned}
& \mathrm{V}(\mathrm{x}) \equiv \mathrm{V}=\mathrm{V}_{\mathrm{R}} \cosh \mathrm{x} \sqrt{\mathrm{zy}}+\mathrm{I}_{\mathrm{R}} \sqrt{\mathrm{z} / \mathrm{y}} \sinh \mathrm{x} \sqrt{\mathrm{zy}}, \\
& \mathrm{I}(\mathrm{x}) \equiv \mathrm{I}=\mathrm{I}_{\mathrm{R}} \cosh \mathrm{x} \sqrt{\mathrm{zy}}+V_{R} \sqrt{\mathrm{y} / \mathrm{z}} \sinh \mathrm{x} \sqrt{\mathrm{zy}} .
\end{aligned}
$$

where $V_{R}$ and $I_{R}$ are the peak values of the receiving end voltage and current, respectively.

The characteristic impedance, $\mathrm{Z}_{\mathrm{O}}$, and the propagation constant, $\gamma$, are defined as

$$
\begin{aligned}
& z_{0} \equiv \sqrt{z / y} \\
& \gamma \equiv \sqrt{z y}=a+j \beta_{o}
\end{aligned}
$$

where $a$ and $\beta_{0}$ are the attenuation constant and the phase constant. respectively. By using equations (18) and (19), equations (16) and (17) become

$$
\begin{aligned}
& V=V_{R} \cosh \gamma x+I_{R} Z_{0} \sinh \gamma x, \\
& I=I_{R} \cosh \gamma x+\left(V_{R} / Z_{0}\right) \sinh \gamma x .
\end{aligned}
$$

* See, for example, Johnson, 으. it., p. 33-34. 
Then the input impedance of a length of line, $\underline{x}$, is given by

$$
\mathrm{Z}=\mathrm{V} / \mathrm{I}=\mathrm{Z}_{\mathrm{o}}\left[\frac{\mathrm{Z}_{\mathrm{R}} \cosh \gamma \mathrm{x}+\mathrm{Z}_{0} \sinh \gamma \mathrm{x}}{\mathrm{Z}_{0} \cosh \gamma \mathrm{x}+\mathrm{Z}_{\mathrm{R}} \sinh \gamma \mathrm{x}}\right] \text {, }
$$

where

$$
Z_{R}=V_{R} / I_{R}
$$

Now for most practical lines employed in resonant applications, the line is constructed with low losses. For simplicity and with good accuracy, these lines are usually treated as "lossless". It is this type of line that is to be emphasized in this work. Hence, the assumption is made that the line is lossless, and this is defined to mean

$$
\begin{aligned}
& r=0, \\
& g=0 ;
\end{aligned}
$$

that is, the series resistance per unit length and the shunt conductance per unit length are zero, where in general.

$$
\begin{aligned}
& z=r+j \omega L, \\
& y=g+j \omega C .
\end{aligned}
$$

The characteristic impedance from (18) becomes a constant equal to the square root of the ratio of the series inductance per unit length, $L$, and the shunt capacitance, per unit length, C,

$$
\mathrm{z}_{\mathrm{o}} \doteq \sqrt{\mathrm{L} / \mathrm{C}}
$$

Similarly,

$$
\gamma \doteq j \beta_{0}=j \omega \sqrt{L C}
$$


from which

$$
\beta_{0}=\omega \sqrt{L C}
$$

and

$$
\begin{aligned}
& V=V_{R} \cos \beta_{0} x+j I_{R} Z_{0} \sin \beta_{0} x, \\
& I=I_{R} \cos \beta_{0} x+j\left(V_{R} / Z_{0}\right) \sin \beta_{0} x . \\
& Z=Z_{0}\left[\frac{Z_{R} \cos \beta_{0} x+j Z_{0} \sin \beta_{0} x}{Z_{0} \cos \beta_{0} x+j Z_{R} \sin \beta_{o} x}\right] .
\end{aligned}
$$

Ordinarily for applications in which large standing waves are desired, the terminating impedance at one end of the line is either zero or infinite because theoretically both of these conditions yield complete reflection. Since the following chapters will be concerned largely with these two cases, viz,

$$
Z_{R}=\left\{\begin{array}{l}
0 \\
\infty
\end{array},\right.
$$

it is expedient to list the appropriate equations.

The Shorted Line $\left(z_{R}=0\right)$. - Taking $Z_{R}$ equal to zero, $(31),(32)$, and (33) become

$$
\begin{aligned}
& \mathrm{V}=j \mathrm{I}_{\mathrm{R}} \mathrm{Z}_{\mathrm{o}} \sin \beta_{\mathrm{o}} \mathrm{x}, \\
& \mathrm{I}=\mathrm{I}_{\mathrm{R}} \cos \beta_{\mathrm{o}} \mathrm{x}, \\
& \mathrm{Z}=\mathrm{j} \mathrm{Z}_{\mathrm{o}} \tan \beta_{\mathrm{o}} \mathrm{x} .
\end{aligned}
$$

The Open Line $\left(Z_{R}=\infty\right)$.- Similarly by taking $Z_{R}$ to be infinite, the equations (31) through (32) become 


$$
\begin{aligned}
& V=V_{R} \cos \beta_{0} x, \\
& I=j\left(V_{R} / Z_{0}\right) \sin \beta_{0} x, \\
& Z=-j Z_{0} \cot \beta_{0} x
\end{aligned}
$$

Power Losses in Line.--Equations (35) through (40) adequately describe the typical resonant properties of low-loss lines except for power considerations. To include the expression for power loss, the method is employed which assumes that the current distribution along the line in the presence of small losses is essentially the same as for the lossless case. * The power may then be obtained to a good approximation by integration as shown below.

The power loss in length of line, $d x$, is

$$
\mathrm{p}=(\mathrm{r} / 2)|\mathrm{I}|^{2} \mathrm{dx}
$$

and the total average power loss in length, $l$, is given by

$$
\mathrm{P}=(\mathrm{r} / 2) \int_{0}^{\ell}|\mathrm{I}|^{2} \mathrm{~d} \mathrm{x} \text {. }
$$

If the actual current distribution, $I(x)$, is replaced by the value given for the lossless case, then for $Z_{R}$ equal to zero,

$$
P_{0}=(r / 2) I_{R}^{2} \int_{0}^{2} \cos ^{2} \beta_{0} x d x .
$$

and for $Z_{R}$ equal to infinity,

$$
P_{\infty}=(r / 2)\left(V_{R} / Z_{0}\right)^{2} \int_{0}^{l} \sin ^{2} \beta_{0} x d x \text {, }
$$

which simplify to the following:

$$
P_{0}=(r / 2) I_{R}^{2}\left[(\ell / 2)+\left(1 / 4 \beta_{0}\right) \sin 2 \beta_{0} \ell\right] \text {, }
$$

* See, Simon Ramo, and John R. Whinnery, Fields and Waves in Modern Radio, New York: John Wiley and Sons, Inc., 1944, p. 248. 


$$
P_{\infty}=(r / 2)\left(V_{R} / Z_{0}\right)^{2}\left[(l / 2)-\left(1 / 4 \beta_{0}\right) \sin 2 \beta_{0} l\right] .
$$

For quarter-wave resonance,

$$
\beta_{0} \ell_{0}=\pi / 2
$$

Then (45) and (46) become

$$
\begin{aligned}
& P_{0}=(r / 2) I_{R}{ }^{2}\left(l_{0} / 2\right)=(r / 2) I_{R}{ }^{2}(\lambda / 8) \\
& P_{\infty}=(r / 2)\left(V_{R} / Z_{0}\right)^{2}\left(\ell_{0} / 2\right)=(r / 2)\left(V_{R} / Z_{0}\right)^{2}(\lambda / 8) .
\end{aligned}
$$

The quarter $\sim$ wave resonator indicated by (47) is normally a uniform line open at one end and shorted at the other. Thus, (48) and (49) are expressions that represent the same line where $I_{R}$ in (48) is the current at the shorted end and $V_{R}$ in (49) is the voltage at the open end. Hence, (48) and (49) may be equated; it follows that the voltage at the open end is equal to the product of the characteristic impedance times the current at the shorted end; that is, with the shorted end as the reference,

$$
\mathrm{V}_{\mathrm{S}}=\mathrm{I}_{\mathrm{R}} \mathrm{Z}_{\mathrm{o}}
$$

The parallel resonant impedance looking into the open end is approximately given by

$$
\mathrm{R}_{\mathrm{p}}=\mathrm{V}_{\mathrm{R}}^{2} / 2 \mathrm{P}_{\infty}=8 \mathrm{Z}_{\mathrm{o}}^{2} / \mathrm{r} \lambda
$$

Summary. - - This chapter presents the differential equations for the general line and gives the general nomenclature and symbolism to be used in the following chapters. In addition, the expressions for the uniform line which are significant to the work that follows are listed for reference.

In the next chapter the analogous expressions for the experimental lines are developed. 


\section{CHAP TER III}

\section{THE EXPONENTIAL LINE}

Introduction.--Following the nomenclature and symbolism established in Chapter II, the theory of the exponential. line is developed and discussed in this chapter. For clarity, only the significant steps are in. cluded in the mathematical development. The major emphasis is on the resonant properties of the lossless line. A detailed discussion of the results is deferred to Chapter VI.

Additional Symbolism for Distinguishing the Exponential Line. $\rightarrow$ In order to distinguish voltage, current, impedance, power, resonant frequency, and resonant length expressions for the exponential line from analogous expressions for the uniform line, an additional subscript " $\mathrm{E}$ " is used when required. Certain quantities are defined by special symbols at various convenient points in the development. Definition of the Exponential Line. - The exponential line is defined as follows:

$$
\begin{aligned}
& z=\left(R_{0}+j \omega L_{0}\right) e^{-2 \delta x} \equiv z_{0} e^{-2 \delta x}, \\
& y=\left(G_{0}+j \omega C_{0}\right) e^{2 \delta x} \equiv y_{0} e^{2 \delta x}
\end{aligned}
$$

where the subscript o denotes values at the origin. The quantity, $\underline{\delta}$, which indicates the impedance taper of the line (and which has the same units as the phase constant, $\beta_{0}$ ), is called the taper constant and is used in the generic sense having both negative and positive values. For positive and negative values of $\underline{\delta}$, the family of exponential. lines are termed positively and negatively tapered lines, respectively. The positive line signifies a decrease, while the negative line indicates an increase, of nominal characteristic impedance with distance from the receiving end. The definitions are illustrated in Fig. 7. 


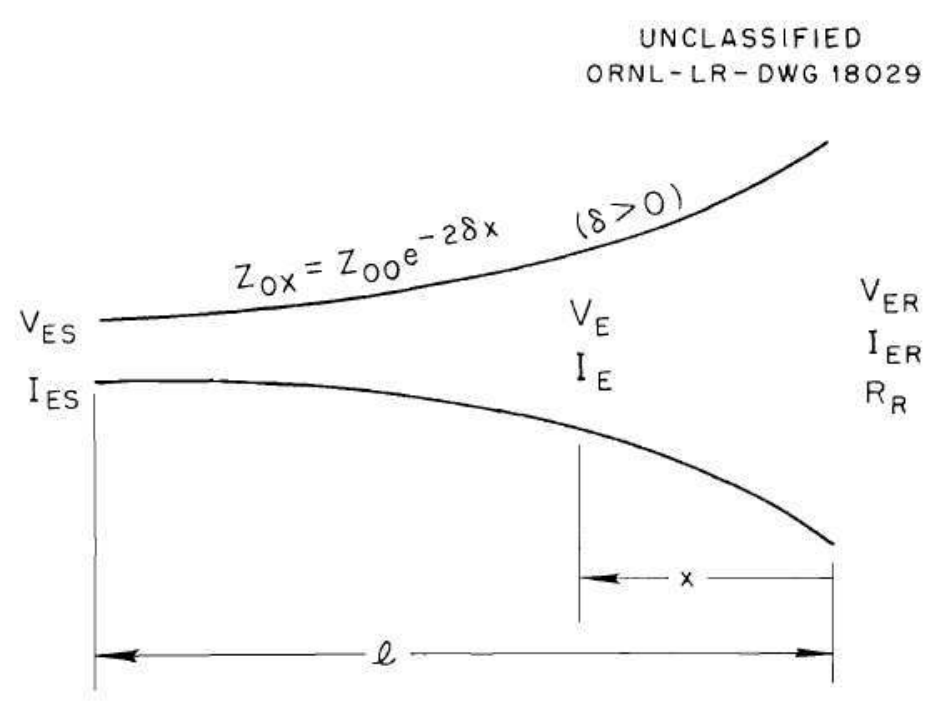

(a)

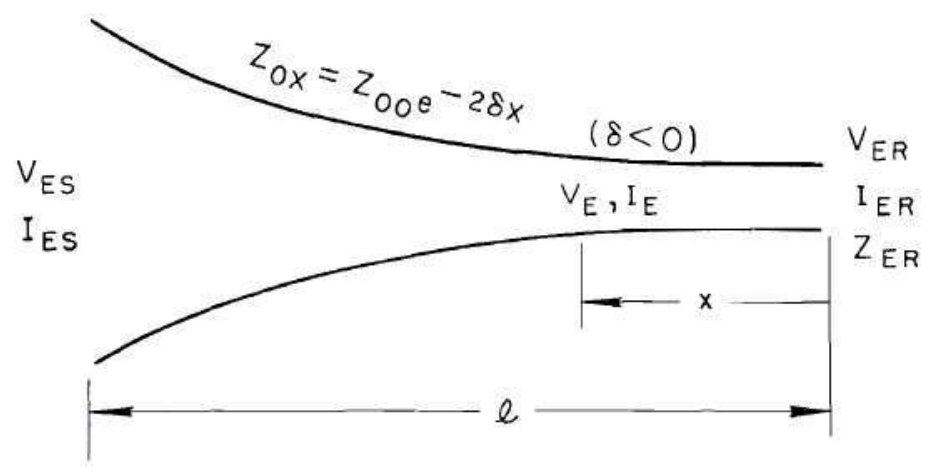

(b)

Fig. 7. (a) The Positively Tapered and

(b) the Negatively Tapered Exponential Line. 
Thus, from (52) and (53), the exponential line is seen to be characterized by a nominal characteristic impedance that varies exponentially with distance, that is,

$$
z_{O E}=\sqrt{z / y}=z_{O O} e^{-2 \delta x}
$$

where

$$
z_{o 0} \equiv{\sqrt{z_{0} / y_{0}}}
$$

Mathematical Development of the Exponential Line - - The differential equations for the exponential line are obtained by substituting (52) and (53) above in (12) and (13) from Chapter II. The resulting equations are

$$
\begin{aligned}
& \mathrm{d}^{2} \mathrm{~V}_{E} / \mathrm{dx^{2 }}+2 \delta d V_{E} / \mathrm{dx}-\gamma^{2} \mathrm{~V}_{E}=0, \\
& \mathrm{~d}^{2} \mathrm{I}_{E} / d \mathrm{x}^{2}-2 \delta d \mathrm{I}_{E} / \mathrm{dx}-\gamma^{2} \mathrm{I}_{E}=0,
\end{aligned}
$$

where

$$
\gamma \equiv \sqrt{z y}=\sqrt{z_{0} y_{0}}
$$

which is seen to be independent of the taper of the line. Since (56) and (57) are homogeneous linear differential equations, the voltage and the current have the following form:

$$
\begin{aligned}
& V_{E}=e^{-\delta x_{(}}\left(C_{1} e^{\Gamma x}+C_{2} e^{-\Gamma x}\right) \\
& I_{E}=e^{\delta x}\left(C_{3} e^{\Gamma x}+C_{4} e^{-\Gamma x}\right)
\end{aligned}
$$

With the boundary conditions,

$$
\begin{aligned}
& V_{E}(0)=V_{R}, \\
& I_{E}(0)=I_{R},
\end{aligned}
$$




$$
\mathrm{z}_{\mathrm{R}}=\mathrm{V}_{\mathrm{R}} / \mathrm{I}_{\mathrm{R}} \text {, }
$$

the integration constants $C_{1}, C_{2}, C_{3}$, and $C_{4}$ become

$$
\begin{aligned}
& C_{1}=\frac{V_{R}(\Gamma-\delta)-z_{O} I_{R}}{2 \Gamma} \\
& C_{2}=\frac{V_{R}(\Gamma+\delta)+z_{o} I_{R}}{2 \Gamma} \\
& C_{3}=\frac{I_{R}(\Gamma+\delta)-y_{0} V_{R}}{2 \Gamma} \\
& C_{4}=\frac{I_{R}(\Gamma-\delta)+y_{0} V_{R}}{2 \Gamma}
\end{aligned}
$$

If equations (63) through (67) are substituted in (59) and (60), the general expressions for $\mathrm{V}_{E}$ and $\mathrm{I}_{E}$ follow,

$$
\begin{aligned}
& V_{E}=\left(V_{R} / \Gamma\right)\left[\Gamma \cosh \Gamma x+\left(\delta+z_{o} / z_{R}\right) \sinh \Gamma x\right] e^{-\delta x}, \\
& I_{E}=\left(V_{R} / \Gamma\right)\left[\left(\Gamma / z_{R}\right) \cosh \Gamma x-\left\{\left(\delta / z_{R}\right)-y_{0}\right\} \sinh \Gamma x\right] e^{\delta x},
\end{aligned}
$$

and

$$
z_{E}=V_{E} / I_{E}=\left[\begin{array}{l}
\Gamma \cosh \Gamma x+\left(\delta+z_{o} / z_{R}\right) \sinh r x \\
\left.\left(\bar{r} / Z_{R}\right) \operatorname{coshrx}-\left(\delta / Z_{R}\right)-y_{o}\right) \sinh T x
\end{array}\right] e^{-2 \delta x_{(70)}}
$$

where

$$
\Gamma=\sqrt{\delta^{2}+\gamma^{2}} \text {. }
$$

At this point the general expressions (68) through (71) may be simplified for study by assuming a lossless line. 
The Lossless Exponential Line.--The following discussion considers the line to be lossless for purposes of obtaining simplified expressions of current, voltage, and input impedance, that is,

$$
\begin{aligned}
& \mathrm{R}_{\mathrm{o}}=0, \\
& \mathrm{G}_{\mathrm{o}}=0,
\end{aligned}
$$

Thus by using (72) and (73) in (52), (53), (55), (58), and (71), the following equations result:

$$
\begin{aligned}
& z_{0}=j \omega L_{0} \equiv j x_{0}, \\
& y_{0}=j \omega C_{0} \equiv j B_{0}, \\
& z_{O O}=\sqrt{L_{0} / C_{0}}, \\
& \gamma=j \omega \sqrt{L_{0} / C_{0}}=j \omega / v^{\prime}, \\
& T=\sqrt{\delta^{2}-\omega^{2} L_{0} C_{0}},
\end{aligned}
$$

where $\mathrm{v}^{\prime}$ is the velocity of propagation along a uniform line (with a series inductance per unit length, $L_{0}$, and a shunt capacitance per unit length, $\mathrm{C}_{\mathrm{O}^{\prime}}$ ) and is given by

$$
v^{i}=1 / \sqrt{L_{0} C_{0}}
$$

From (78), it is seen that $\Gamma$ may be either real or imaginary, according to the value of the quantity

$$
\delta^{2}-\omega^{2} L_{0} C_{0}
$$

A study of equations (59) and (60) shows that, if

$$
\delta^{2}>\omega^{2} L_{0} C_{0}
$$


the attenuation of the voltage and current with distance is greater than if

$$
\delta^{2}<\omega^{2} L_{0} C_{0}
$$

Hence, for this work, interest is confined to the latter case since, for resonant operation, minimum loss is desired. The boundary between the two cases is given by

$$
\delta^{2}=\omega_{c}^{2} L_{0} C_{0}
$$

so that a critical frequency is defined as follows:

$$
f_{c}=(|\delta| / 2 \pi)\left(1 / \sqrt{L_{0} C_{o}}\right)=(|\delta| / 2 \pi) v^{\prime} .
$$

This is the quantity called the "cut-off" frequency used by Burrows* in showing that the exponential line behaves as a high-pass impedance transforming filter. However, as Johnson ${ }^{* * *}$ points out, this term is misleading since energy is transmitted to the load at frequencies below $f_{c}$ unless the line is very long. Hence, the term critical frequency is used here. In addition, the following definitions are employed for convenience:

$$
\begin{aligned}
& T \equiv j \beta \\
& \beta \equiv \sqrt{\beta_{0}{ }^{2}-\delta^{2}} \\
& \beta_{0} \equiv \sqrt{\omega^{2} L_{0} C_{0}}=\omega / v^{\prime} .
\end{aligned}
$$

\footnotetext{
*Burrows, op. cit., p. 559.

***Johnson, op. cit., p. 205.
} 
When (63) and (85) are substituted in (68), (69), and (70), the general equations reduce to the following:

$$
\begin{aligned}
& V_{E}=V_{R}\left[\cos \beta x+(1 / \beta)\left(\delta+\left(z_{o} / z_{R}\right)\right) \sin \beta x\right] e^{-\delta x}, \\
& I_{E}=I_{R}\left[\cos \beta x-(1 / \beta)\left\{\delta-\left(y_{o} / z_{R}\right) \sin \beta x\right] e^{\delta x},\right. \\
& Z_{E}=Z_{R}\left[\frac{\cos \beta x+(1 / \beta)\left(\delta+z_{o} z_{R}\right) \sin \beta x}{\cos \beta x-(1 / \beta)\left(\delta-y_{o} Z_{R}\right) \sin \beta x}\right] e^{-2 \delta x} .
\end{aligned}
$$

Thus, (88), (89), and (90) are the basic equations of the lossless exponential line terminated in an arbitrary impedance, $Z_{R^{\circ}}$ In order to understand more clearly the resonant properties of this type of line, the expressions for two particular terminations are developed. These two terminations are called the open $\left(Z_{R}\right.$ equals infinity) and the shorted $\left(Z_{R}\right.$ equals zero) lines, respectively。 The Open Line $\left(Z_{R}=\infty\right)$. - For this case, the basic equations, (88), (89), and (90) become

$$
\begin{aligned}
& V_{E}=V_{R}(\cos \beta x+(\delta / \beta) \sin \beta x] e^{-\delta x}, \\
& I_{E}=V_{R}\left[\left(y_{0} / \beta\right) \sin \beta x\right] e^{\delta x} \\
& Z_{E}=\left[\frac{\cos \beta x+(\delta / \beta) \sin \beta x}{\left(y_{0} / \beta\right) \sin \beta x}\right] e^{-2 \delta x} .
\end{aligned}
$$

An additional expression, the power loss, may be calculated by the same method introduced in Chapter II for the uniform line. Thus, the power los in length of line, dx, is

$$
p=\left(|I|^{2} / 2\right) R d x
$$

The resistance per unit length, $R$, is assumed constant so that the expression for power loss for the exponential line may be compared in 
Chapter VI with that of the uniform line. As will be shown in Appendix I, the above assumption is easily met by using a parallel-strip ${ }^{*}$ line. Then from (92) and (94), the average power loss in length, $l$, is

$$
\begin{aligned}
P_{E}=\left(V_{R} B_{0} / \beta\right)^{2}(R / 2) & \int_{0}^{l} e^{2 \delta x_{s^{2}}{ }^{2} \beta x d x,} \\
P_{E}=\left(V_{R} B_{0} / \beta\right)^{2}(R / 8) & {\left[\begin{array}{lll}
2 \delta l & 1+\delta / \beta_{0}^{2} \\
& -(\delta \cos 2 \beta l+\beta \sin 2 \beta l) e^{2 \delta l} / \beta_{0}
\end{array}\right] . }
\end{aligned}
$$

Next, the resonant properties of the open line are investigated by obtaining the expressions for the resonant lengths of line analog ous to the quarter-wave and half-wave resonant conditions of the uniform line, that is,

$$
\mathrm{Z}_{\mathrm{E}}=0
$$

and

$$
Z_{E}=\infty \quad,
$$

respectively. Thus for quarter-wave resonance, the following equation results from (93) and (97):

$$
\cos \beta a+(\delta / \beta) \sin \beta a=0
$$

or

$$
\cot \beta a=-\delta / \beta
$$

For a fixed frequency, the resonant lengths for resonances analogous to those of a uniform line, viz,

$$
\lambda / 4,3 \lambda / 4,5 \lambda / 4 \text {, etc....., (2m+1) } \lambda / 4
$$

*Federal Telephone and Radio Corporation, Reference Data for Radio Engineers, 3rd Ed., New York: American Book-Knickerbocker Press, Inc., p. 325. 
are

$$
\begin{gathered}
a_{m}=(1 / \beta)[(m+1) \pi-\theta] \\
(m=0,1,2,3, \ldots)
\end{gathered}
$$

where

$$
\theta=\cot ^{-1} \delta / \beta
$$

Conversely, for a fixed length, a, the corresponding resonant frequencies may be obtained from (100) by an approximate method which will be described later in this chapter.

Similarly, for half-wave resonances, (93) and (98) yield

$$
\cot \beta b+\delta / \beta=\infty
$$

from which, for a fixed frequency,

$$
\begin{aligned}
& \mathrm{b}_{\mathrm{n}}=\mathrm{n} \pi / \beta, \\
& (\mathrm{n}=0,1,2,3, \ldots \cdot),
\end{aligned}
$$

for the resonances indicated as

$$
\lambda / 2,2 \lambda / 2,3 \lambda / 2 \text {, etc...., n } \lambda / 2
$$

For a fixed length, $\underline{b}$, the corresponding resonant frequencies are

$$
\beta_{n}=n \pi / b \quad .
$$

Next, the shorted line is treated in a similar manner. The Shorted Line $\left(Z_{R}=0\right)$. - - For this case, equations (88), (89), and (90) become

$$
V_{E}=I_{R}\left[\left(z_{0} / \beta\right) \sin \beta x y e^{-\delta x}\right.
$$




$$
\begin{aligned}
& I_{E}=I_{R}(\cos \beta x-(\delta / \beta) \sin \beta x) e^{\delta x} \\
& Z_{E}=\left[\frac{\left(z_{O} / \beta\right) \sin \beta x}{\cos \beta x-(\delta / \beta) \sin \beta x}\right] \mathrm{e}^{-2 \delta x} .
\end{aligned}
$$

The expression for power loss may be obtained by the same meth od used for (95) and is found to be

$$
\begin{aligned}
P_{E} & =\left(I_{R} \beta_{0} / \beta\right)^{2}(R / 8 \delta)\left[e^{2 \delta l}-1\right. \\
& -\left\{\delta\left(\delta^{2}-\beta^{2}\right)(\delta \cos 2 \beta l+\beta \sin 2 \beta l)\right. \\
& +4 \beta \delta(\delta \sin 2 \beta l-\beta \cos 2 \beta l)\} e^{2 \delta l} / \beta_{0}^{2} \\
& \left.-\delta^{2} / \beta_{0}^{2}\right] .
\end{aligned}
$$

The quarter-wave and half-wave resonant conditions are the reverse of those for the open line, viz,

$$
\mathrm{Z}_{\mathrm{E}}=\infty
$$

and

$$
\mathrm{Z}_{\mathrm{E}}=0,
$$

respectively. By using (108) and (110), the following equation is obtained:

$$
\cos \beta A-(\delta / \beta) \sin \beta A=0
$$

or

$$
\cot \beta A=\delta / \beta .
$$


For a fixed frequency, the resonant lengths are

$$
\begin{aligned}
& A_{m}=(1 / \beta)(m \pi+\theta) \\
& (m=0,1,2,3, \ldots)
\end{aligned}
$$

for the resonances,

$$
\lambda / 4,3 \lambda / 4,5 \lambda / 4, \ldots,(2 m+1) \lambda / 4
$$

where $\theta$ is given by (102). The corresponding resonant frequencies, for a fixed length, A, are obtained by solving (113) by approximate methods.

Similarly, for the half-wave resonances,

$$
\lambda / 2,2 \lambda / 2,3 \lambda / 2, \ldots, \mathrm{n} \lambda / 2
$$

the condition is

$$
\cot \beta B=\infty,
$$

and for a fixed frequency the resonant lengths are

$$
\mathrm{B}_{\mathrm{n}}=\mathrm{n} \pi / \beta \text {. }
$$

Conversely, for a fixed length. $\underline{B}$, the resonant frequencies are

$$
\beta_{n}=n \pi / B \text {. }
$$

The mathematical development of the essential expressions for the lossless exponential line is concluded with equation (117). Illustrations of some of these results are presented below in order to give a clear physical picture of the characteristics of this type of line. 
Input Impedance vs Length. - - If equation (101) for the open line is reexamined considering $\delta$ to be positive, then, since $\beta \mathrm{a}$ is intrinsically positive, the zeros of the input impedance are

$$
\frac{\pi-\theta}{\beta}, \frac{2 \pi-\theta}{\beta}, \ldots,
$$

and from (104) the infinities of the impedance are

$$
0, \frac{\pi}{\beta}, \frac{2 \pi}{\beta}, \ldots
$$

Similarly for $\delta$ negative, the zeros and infinities are

$$
\frac{\theta}{\beta}, \frac{\pi+\theta}{\beta}, \frac{2 \pi+\theta}{\beta}, \ldots
$$

and

$$
0, \frac{\pi}{\beta}, \frac{2 \pi}{\beta}, \ldots
$$

respectively. After being rearranged as follows:

$$
z_{E}=\left(\beta e^{-2 \delta x} / y_{0}\right)(\cot \beta x+\delta / \beta)
$$

equation (93) is sketched with the use of the zeros and infinities in Fig. 8, for positive and negative $\delta$. Both the taper constant and the frequency ${ }^{*}$ are held constant for each curve.

Next, the shorted line is treated in a similar manner. Thus, from equation (114) for the shorted line with $\delta$ positive, the zeros are

$$
0, \frac{\pi}{\beta}, \frac{2 \pi}{\beta}, \ldots
$$

\footnotetext{
* The frequency is understood to be above the critical frequency.
} 


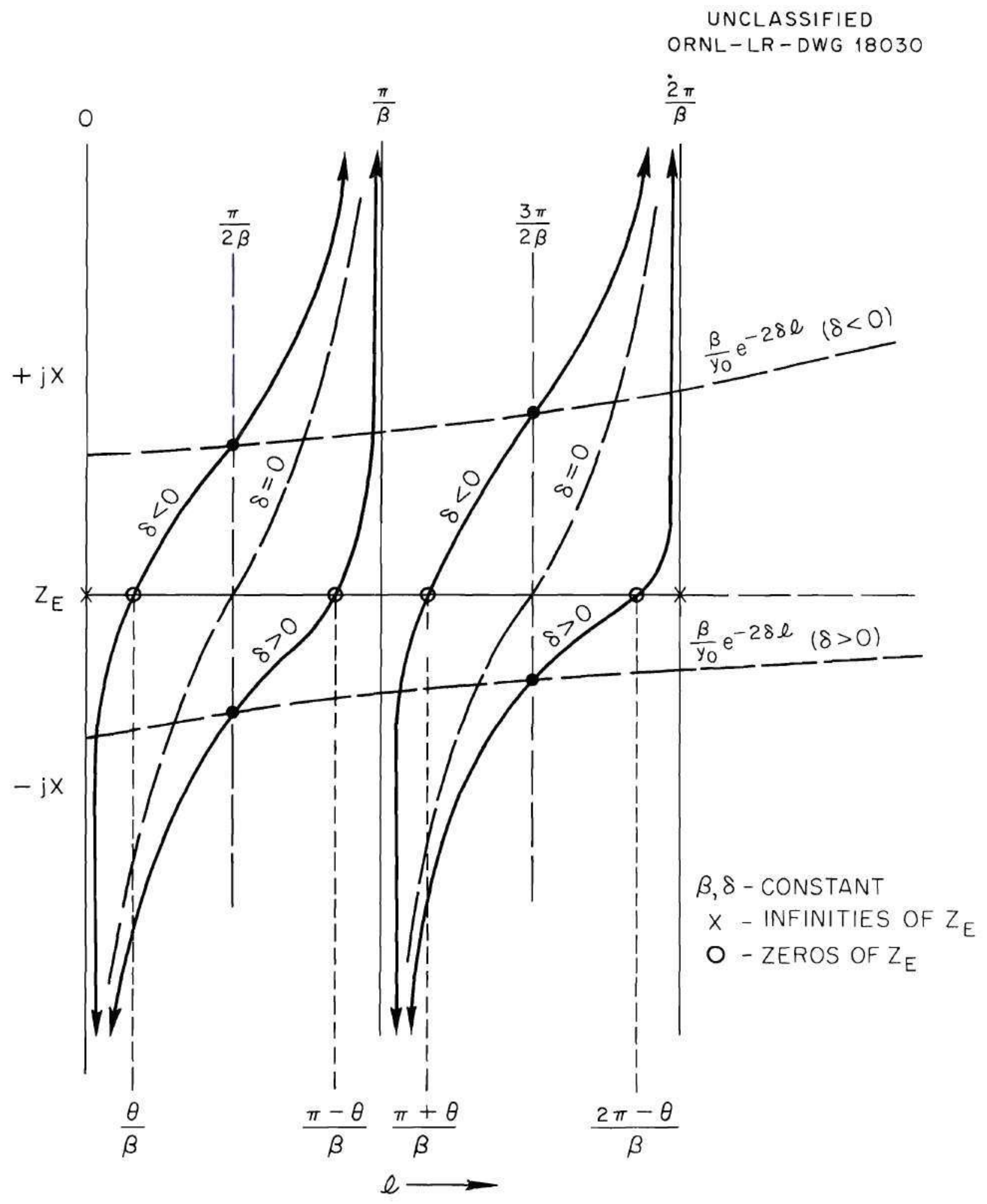

Fig. 8. A Sketch of the Input Impedance vs Length for the Open Line 
and the infinities are

$$
\frac{\theta}{\beta}, \frac{\pi+\theta}{\beta}, \frac{2 \pi+\theta}{\beta}, \ldots
$$

In a like manner for $\delta$ negative, the zeros and infinities are

$$
0, \frac{\pi}{\beta}, \frac{2 \pi}{\beta}, \ldots \text {, }
$$

and

$$
\frac{\pi-\theta}{\beta}, \frac{2 \pi-\theta}{\beta}, \ldots
$$

respectively. When the above information for the shorted line is used, Fig. 9 shows a sketch of equation (108), for both $\delta$ positive and $\delta$ negative, rearranged as follows:

$$
z_{E}=\left(z_{0} e^{-2 \delta x / \beta)}[(1 / \cot \beta x)-\delta / \beta] .\right.
$$

Again the taper constant and the frequency are held constant.

Now if the taper constant and the length are held constant, the input impedance becomes a function of frequency. This topic is considered in the next section.

Input Impedance vs Frequency...-Since equations (118) and (119) are not easily sketched when frequency is the variable, two pairs of curves for the shorted line are plotted for a particular set of conditions. Only the shorted line is so illustrated since the characteristics of the open line are similar. Fig, 10 shows the input impedance vs frequency of a shorted line for the particular set of conditions, viz, the length is held at 16 feet and, two pairs of values are used for $\bar{\delta}$, that is,

$$
\delta= \pm 0.031 \text { radians per foot }
$$

and

$$
\delta= \pm 0.062 \text { radians per foot }
$$




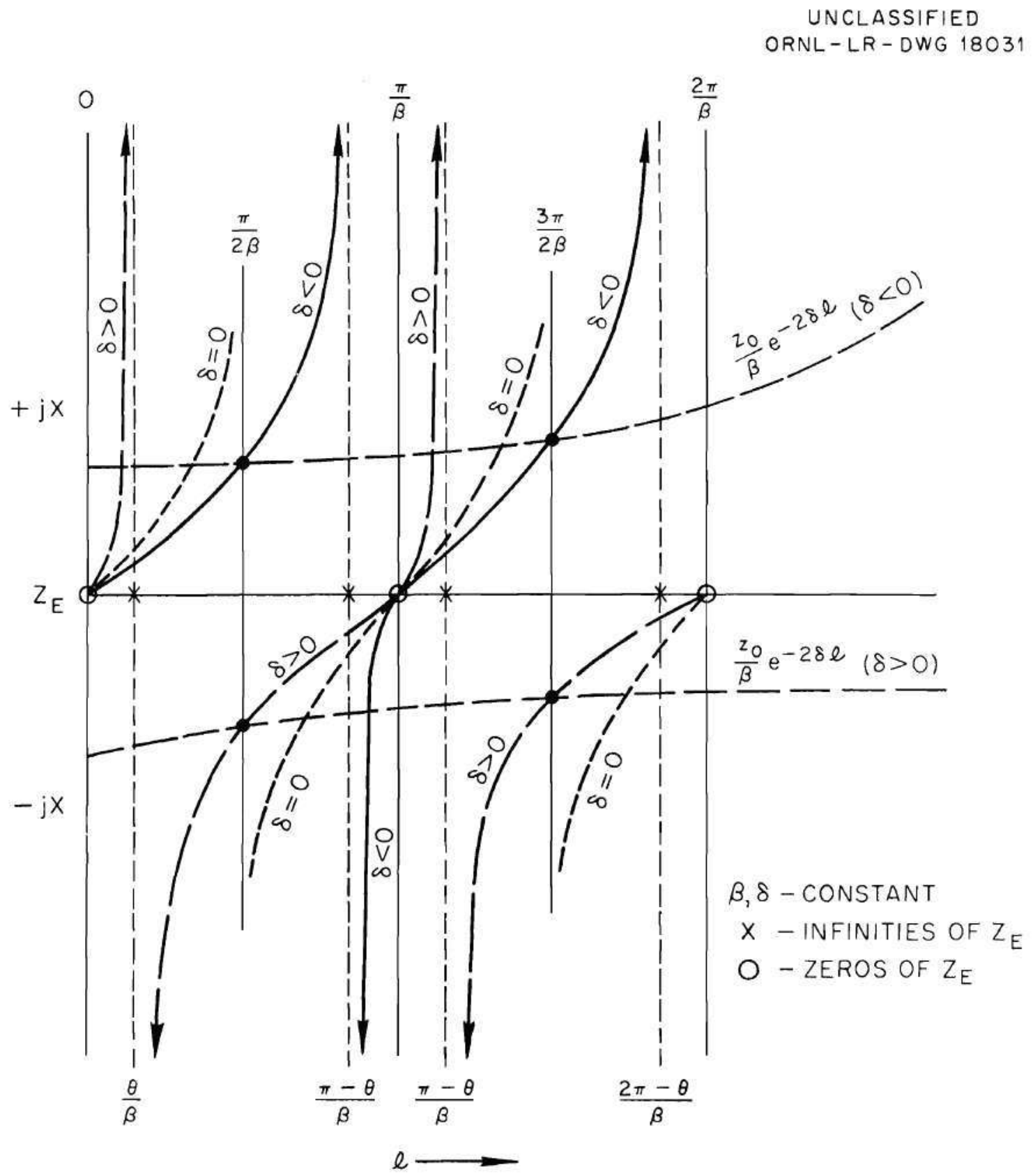

Fig. 9. A Sketch of the Input Impedance vs Length for the Shorted Line 
UNCLASS'FIED

ORNL - LR - DWM 18032

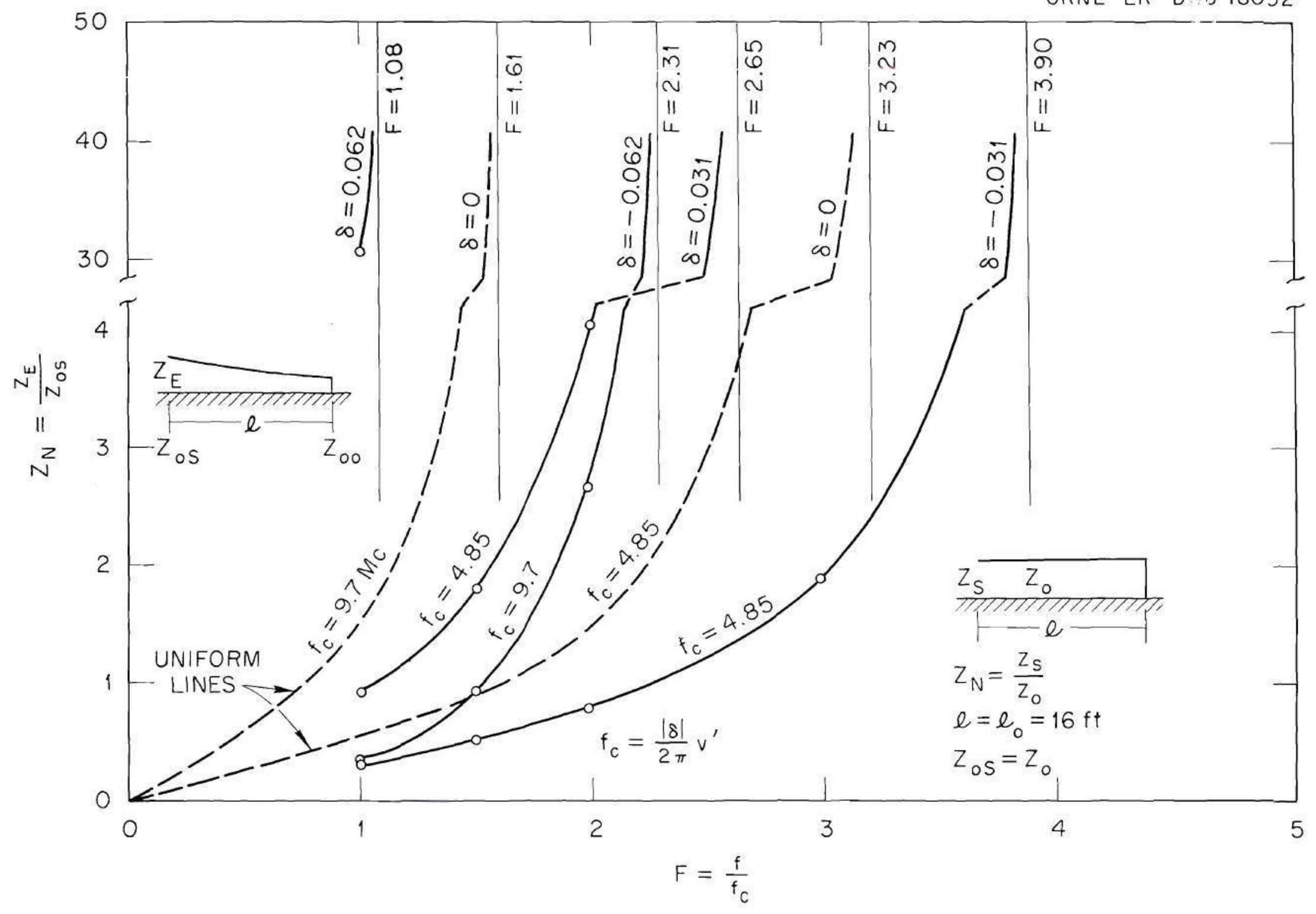

Fig. 10. Normalized Input Impedance vs Normalized Frequency of the Shorted Exponential Line 
In addition, all four lines have the same nominal characteristic impedance at the open end. Normalized scales are employed where the ordinate is the input impedance divided by the nominal characteristic impedance at the open end, $Z_{\text {oS }}$, and the abscissa is the frequency divided by the critical frequency, $f_{c}$. Fig. 11 presents the same data with a direct-frequency scale.

In the next section the quarter-wave resonance condition given by (113) is discussed further.

Quarter-Wave Resonance vs Taper for the Shorted Line.--Equation (113) may be rearranged as follows:

$$
\cot \sqrt{\left(\beta_{0} A_{1}\right)^{2}-\left(\delta A_{1}\right)^{2}}=\delta A_{1} / \sqrt{\left(\beta_{0} A_{1}\right)^{2}-\left(\delta A_{1}\right)^{2}}
$$

by the use of equation (86). Thus, (120) is a function of $\beta_{0} A_{1}$ and $\delta_{A_{1}}$ and is of the form

$$
\tan \mathrm{W}=\mathrm{W} / \mathrm{D}
$$

where

$$
w=\imath\left(p_{0} A_{1}\right)^{2}-\left(\delta_{A_{1}}\right)^{2}
$$

and

$$
D=\delta_{A_{1}}
$$

Also, from (54)

$$
\delta_{A_{1}}=(1 / 2) \log _{e}\left(z_{o o} / z_{o S}\right)
$$

Hence, equation (121) may be solved by approximate methods for $\beta_{0} A_{1}$ as a function of $\mathrm{Z}_{0 \mathrm{O}} / \mathrm{Z}_{\mathrm{oS}}$, the ratio of the nominal characteristic impedances at the ends of the resonant line. 


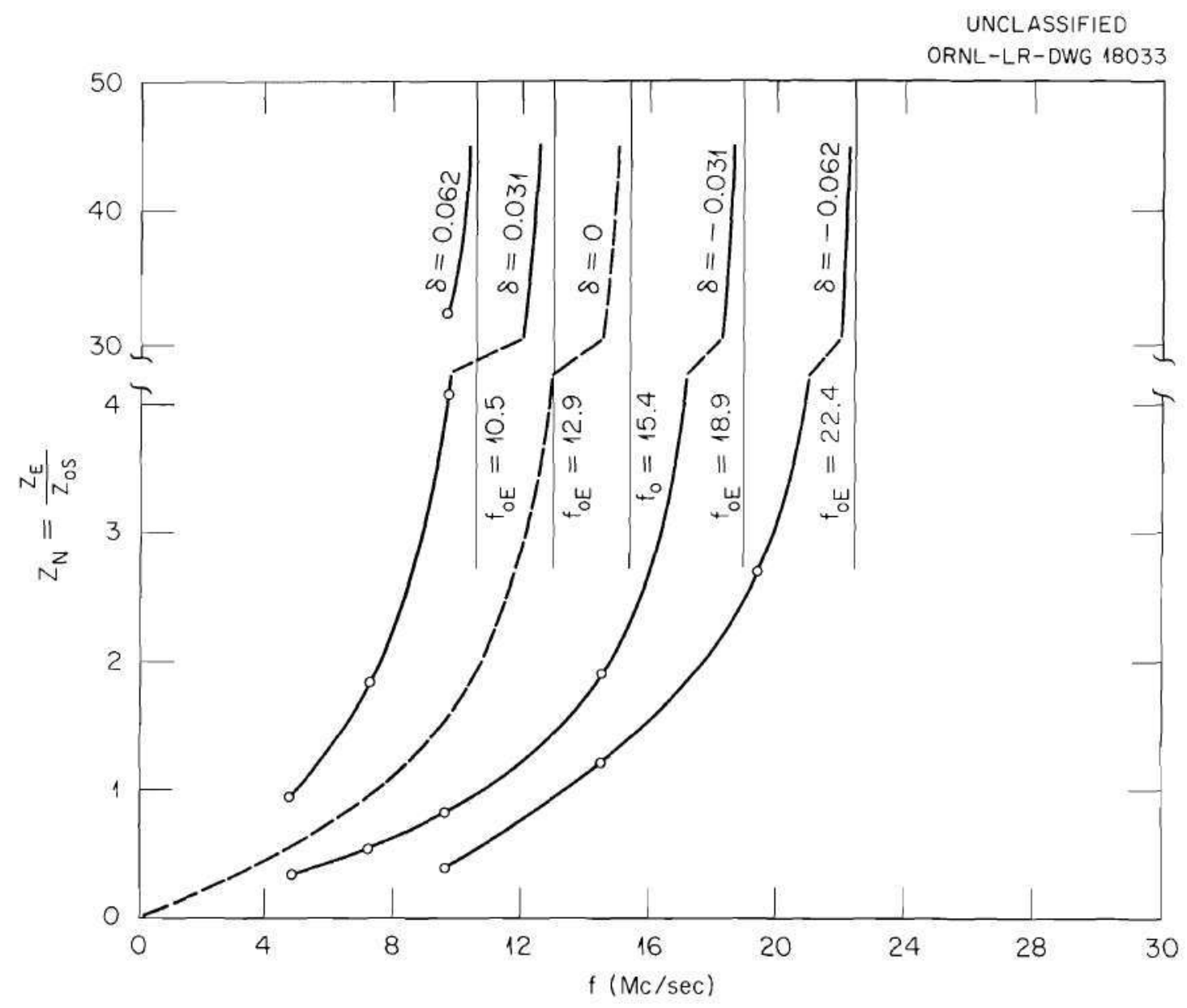

Fig. 11. Normalized Input Impedance vs Frequency of the Shorted Exponential Line. 
Fig. 12 is a composite plot of

$$
\frac{3_{0} A_{1}}{\pi / 2} \text { vs } z_{o o} / Z_{o s}
$$

for $\delta$ negative, and

$$
\frac{\beta_{0} A_{1}}{\pi / 2} \text { vs } Z_{o S} / Z_{0 o}
$$

for $\delta$ positive, so that both ratios, $Z_{o o} / Z_{o S}$ and $Z_{o S} / Z_{o o}$, range from zero to one. It is to be recalled that the quarter-wave resonant condition for the uniform line is

$$
\beta_{0} l_{0}=\pi / 2
$$

Thus, where $\beta_{\mathrm{oE}}$ is used to distinguish the exponential line,

$$
\frac{\beta_{o E^{A}} A_{1}}{\pi / 2}=\frac{\beta_{o E^{A_{1}}}}{\beta_{0} l_{0}},
$$

so that for

$$
\mathrm{A}_{1}=l_{\mathrm{o}}
$$

(that is, the resonant exponential. line is equal in length to the resonant uniform line),

$$
\frac{\beta_{o E^{A}}}{\beta_{o} l_{0}}=f_{o E} / f_{o} .
$$

Then,

$$
\frac{\beta_{o E^{A}}}{\pi / 2}=g\left(Z_{o o} / Z_{o S}\right)
$$




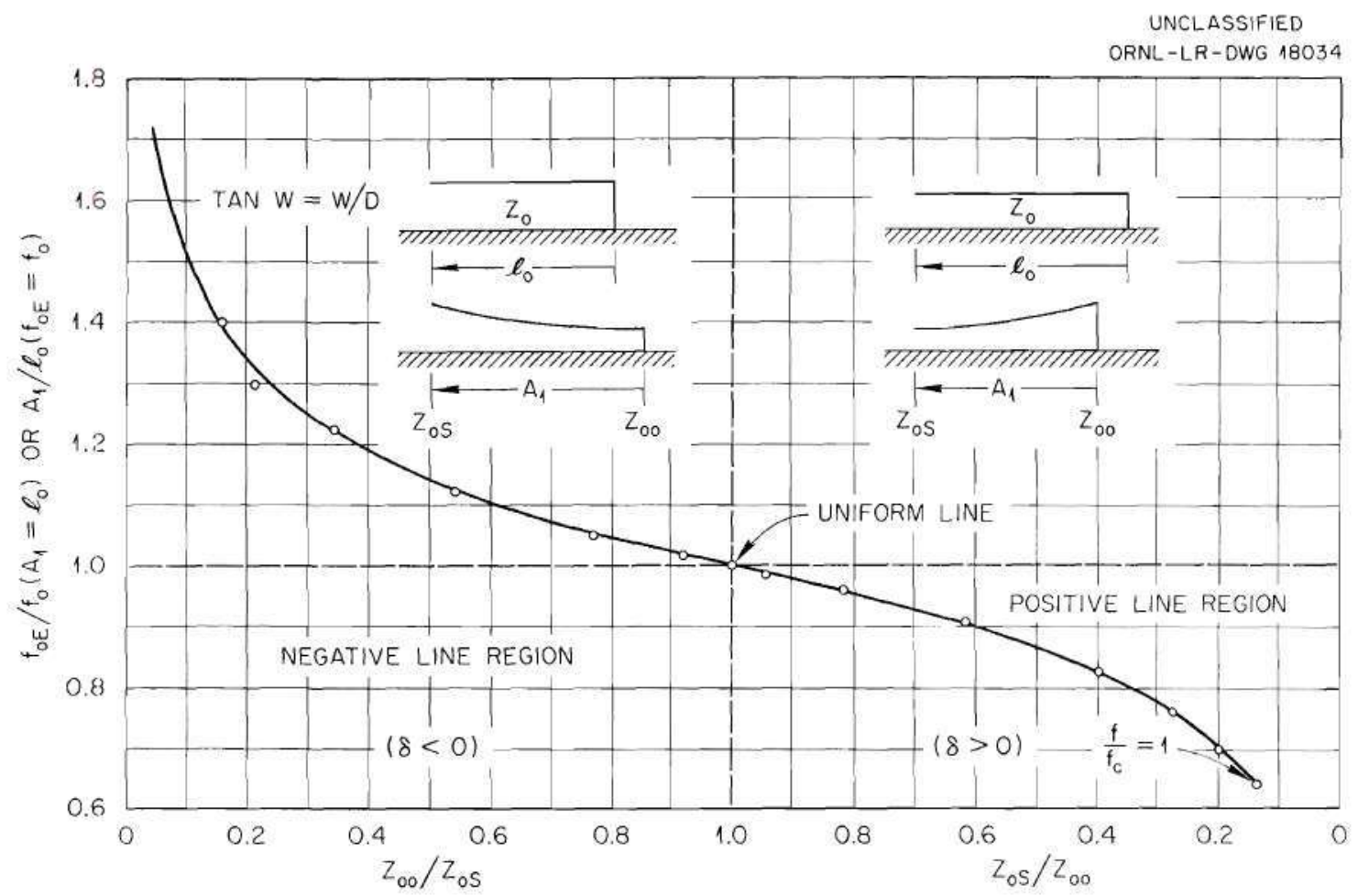

Fig. 12. Quarter-Wave Resonance vs Taper of the Lossless Exponential Line. 
is either a plot of

$$
f_{O E} / f_{O} \quad \text { vs } Z_{O S} / Z_{\infty}
$$

or, for

$$
f_{O E}=f_{O} \quad,
$$

a plot of

$$
A_{1} / k, \quad \text { vi } Z_{o S} / Z_{\infty} .
$$

Hence, the curve in Fig. 12 may be used for either purpose.

Power Loss of the Quarter-Wave Resonant Line $\left(Z_{R}=0\right)$. - - The curve in Fig. 12 may be used with equation (109) and equation (48) from

Chapter II to outain the ratio of power loss of an exponential line to that of a uniform line vhere both lines are in quarter-wave resonance operating at the same trequency.

The expression for the ratio of power loss for the two lines is obtained by substituting (113) in (109) yielding

$$
P_{O E}=\left(I_{R}^{2} R / 8 \delta\right)\left(e^{2 \delta A_{1}}-1-4 \delta^{2} / \beta_{0}^{2}\right)
$$

and subsequently taking the ratio of (131) to (48). Thus,

$$
\mathrm{P}_{\mathrm{oE}} / \mathrm{P}_{\mathrm{o}}=\frac{\left(\mathrm{I}_{\mathrm{R}}^{2} \mathrm{R} / 8 \delta\right)\left(\mathrm{e}^{\left.2 \delta \mathrm{A}_{1}-1-4 \delta^{2} / \beta_{\mathrm{o}}^{2}\right)}\right.}{\left(\mathrm{I}_{\mathrm{R}}^{2} \mathrm{r} \ell_{\mathrm{o}} / 4\right)} .
$$

However, for the exponential line,

$$
I_{E R}=\beta V_{E S} e^{\delta A_{1} / X_{0} \sin \beta A_{1}}
$$


from (106) and (113), and, for the uniform line

$$
I_{R}=V_{S} / Z_{0}
$$

Thus,

$$
P_{o E} / P_{0}=\frac{\left(V_{E S} \beta e^{\delta A_{1 / X}} \sin \beta A_{1}\right)^{2}(R / 8 \delta)\left(e^{\left.2 \delta A_{1}-1-4 \delta^{2} / \beta_{0}^{2}\right)}\right.}{\left(V_{S} / Z_{0}\right)^{2}\left(r \ell_{0} / 4\right)} .
$$

where

$$
\sin \beta A_{1}=\beta / \sqrt{\delta^{2}+\beta^{2}},
$$

and from (86)

$$
\beta_{0}^{2}=\beta^{2}+\delta^{2}
$$

Also, from (74), (87), and (137)

$$
\left(\beta_{0} / x_{0}\right)^{2}=1 / z_{00}^{2}
$$

Thus, after multiplying numerator and denominator oy $\mathrm{A}_{1}$

$$
\begin{array}{r}
P_{o E} / P_{0}=\left[\frac{\left(V_{E S} Z_{0}\right)^{2}(R)\left(A_{1}\right)\left(e^{\left.-2 \delta A_{1}\right)}\right.}{\left(V_{S} Z_{o S}\right)^{2}(r)\left(l_{0}\right)\left(2 \delta A_{1}\right)}\right] \\
\cdot\left[e^{2 \delta_{A_{1}}-1-4\left(\delta_{A_{1}} / \beta_{0} A_{1}\right)^{2}}\right]
\end{array}
$$

But

$$
\beta_{0}=\pi / 2 \ell_{0} .
$$


So, finally, the ratio of power loss is

$$
\begin{gathered}
P_{O E} / P_{0}=\left[\frac{\left(V_{E S} Z_{0}\right)^{2}(R)\left(A_{1}\right)\left(e^{\left.-2 \delta A_{1}\right)}\right.}{\left.\left(V_{S} Z_{O S}\right)^{2(r)\left(\ell_{0}\right)\left(2 \delta A_{1}\right)}\right]}\right. \\
\cdot\left[e^{\left.2 \delta A_{1}-1-4\left(2 \delta_{A_{1}} / \pi\right)^{2}\left(\ell_{0} / A_{1}\right)^{2}\right]}\right.
\end{gathered}
$$

At this point, (140) may be modified to approximate the cyclotron problem for comparison of the two lines represented. The basis for comparing the lines is as follows:

1. Since the accelerating voltage for the cyclotron is a primary parameter, the two lines should have the same voltage at the open end.

2. Since the spacing of the accelerating electrodes is very limited so that a wide choice of values for the nominal characteristic impedance at the open end is not possible, the two lines should have the same nominal characteristic impedance at the open end.

Hence,

$$
\mathrm{v}_{\mathrm{ES}} / \mathrm{v}_{\mathrm{S}}=\mathrm{Z}_{\mathrm{O}} / \mathrm{Z}_{\mathrm{OS}}=1
$$

In addition, for convenience in comparison, let

$$
\mathrm{R} / \mathrm{r}=1
$$

Then (140) becomes

$$
\mathrm{P}_{\mathrm{oE}} / \mathrm{P}_{\mathrm{O}}=\frac{\left(\mathrm{A}_{1}\right)\left(\mathrm{e}^{-2 \delta_{\mathrm{A}_{1}}}\right)}{\left(\ell_{\mathrm{O}}\right)\left(2 \delta_{\mathrm{A}_{1}}\right)}\left[\mathrm{e}^{\left.2 \delta_{\mathrm{A}_{1}}-1-4\left(2 \delta_{\mathrm{A}_{1}} / \pi\right)^{2}\left(\ell_{0} / \mathrm{A}_{1}\right)^{2}\right]}\right.
$$


which is a function of $\mathrm{A}_{1} / \ell_{\mathrm{O}}$ and $\mathrm{z}_{\mathrm{OS}} / \mathrm{z}_{\mathrm{Oo}}$.

Thus, equation (144) may be used in conjunction with Fig. 12 to obtain the power ratio curve

$$
\mathrm{P}_{\mathrm{OE}} / \mathrm{P}_{\mathrm{O}} \text { vs } \mathrm{Z}_{\mathrm{oS}} / \mathrm{Z}_{\mathrm{Oo}}
$$

Fig. 13 shows a plot of this equation where the curve is obtained by picking a value of $\mathrm{A}_{1}$ and obtaining the corresponding value of $\mathrm{Z}_{o S} / \mathrm{Z}_{\infty}$ (or the reciprocal) from Fig. 12 for the condition that both lines are in quarter-wave resonance at the same frequency. Summary. - - The methods of ordinary transmission line theory are employed in this chapter to present a mathematical development of the general equations which describe the exponential line. These equations (68), (69), and (70) are applied to the case of the lossless line in which the series resistance and the shunt conductance are zero. The result. ing expressions (88), (89), and (90) are considerably simplified and are better suited for detailed study. The power losses are obtained by as suming that the current distribution given for the lossless case is not significantly altered in the presence of small losses such that the power loss of a low-loss line is given approximately by integrating the product of the resistance and the square of the (rms) current (for the lossless case) over the length of line involved. It is to be noted that little is sacrificed by emphasizing the lossless line since the results are typical for the majority of the lines used in resonant applications. That is to say, low-loss lines very closely approximate the characteristics of lossless lines. For lines with excessive losses, the general equations may be used.

In addition to the development of mathematical expressions, curves of input impedance vs length, input impedance vs frequency, quarter-wave resonance vs taper, and power loss vs taper are presented for the purpose of comparing the characteristics of the exponential line with analogous properties of other lines. A detailed discus sion of these properties in conjunction with the properties of the uniform and linear lines is given in Chapter VI. 


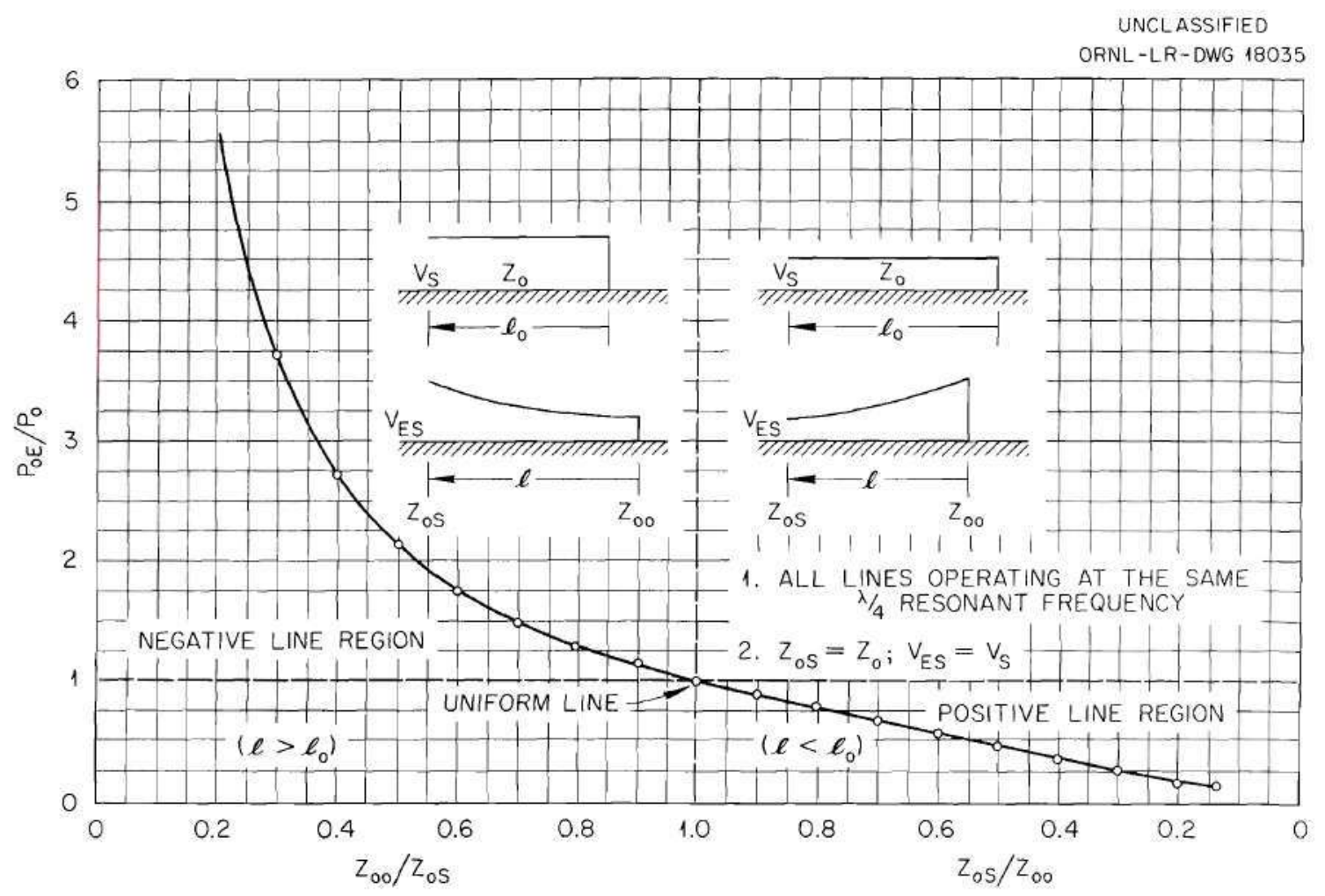

Fig. 13. Power Loss Ratio vs Taper of the Quarter-Wave Exponential Line. 
The results in this chapter are limited to transmission lines whose change in spacing with respect to length along the line is small. A detailed discussion of these limitations is deferred to Chapter VI so that those of the linear line, which are similar, may be treated at the same time. 


\section{CHAPTER IV}

\section{THE LINEAR LINE}

Introduction.-- In this chapter, the theory of the linear line, paralleling the treatment of the exponential line in Chapter III, is developed. Again the emphasis is on the resonant properties of the lossless line. A discussion of the results is postponed to Chapter VI. Additional Symbolism for Distinguishing the Linear Line. --Analogous to the case of the exponential line, an additional subscript " $L$ " is used when required to distinguish voltage, current, impedance, power, resonant frequency, and resonant length expressions for the linear line from similar expressions for the uniform line. Definition of the Linear Line. - - The linear line is defined as follows:

$$
\begin{aligned}
& z=\left(R_{0}+j \omega L_{0}\right)[1-F(x / k)]=L_{0}[1(k / k)] \\
& y=\left(G_{0}+j \omega C_{0}\right)\left[(1 /\{1 \mp(x / k)]\}=y_{0}[y /\{1 F(x / k)\}]\right.
\end{aligned}
$$

The quantity $\mathrm{k}$ which is inversely proportional to the impedance taper of the line, is called the taper constant. Unlike $\delta$ for the exponential line, $\underline{k}$ appears as a positive number for both positively and negatively tapered lines. However, the terms positive and negative line retain the same general meaning established in the last chapter for the exponential line, viz, a positive line signifies an increase, while a negative line indicates a decrease, of nominal characteristic impedance with distance from the sending end of the line.

From (145) and (146) the linear line is observed to be characterized by a nominal characteristic impedance that varies linearly with distance, that is,

$$
z_{o L} \equiv \sqrt{z / y}=Z_{o o[}[1-(x / k)]
$$


for the positive line, and

$$
Z_{o L}=Z_{O o L}[1+(x / k)]
$$

for the negative line, where

$$
z_{\mathrm{oO}} \equiv \sqrt{z_{\mathrm{o}} / \mathrm{y}_{\mathrm{o}}}
$$

Mathematical Development of the Linear Line. - The differential equations for the linear line are obtained by substituting (145) and (146) in (12) and (13) from Chapter II. The resulting equations are

$$
\begin{aligned}
& d^{2} V_{L} / d x^{2}+[1 /(k-x)] d V_{L} / d x-\gamma^{2} V_{L}=0 \\
& d^{2} I_{L} / d x^{2}-[1 /(k-x)] d I_{L} / d x-\gamma^{2} I_{L}=0
\end{aligned}
$$

for the positive line, and

$$
\begin{aligned}
& d^{2} V_{L} / d x^{2}-[1 /(k+x)] d V_{L} / d x-\gamma^{2} V_{L}=0 \\
& d^{2} I_{L} / d x^{2}+\left[(1 /(k+x)] d I_{L} / d x-\gamma^{2} I_{L}=0\right.
\end{aligned}
$$

for the negative line.

At this point a change of variable is introduced for convenience. Let

$$
\mathrm{u}=\mathrm{k}-\mathrm{x}
$$

for (150) and (151), and

$$
\mathrm{u}=\mathrm{k}+\mathrm{x}
$$


for (152) and (153). Then (150), (151), (152), and (153) become

$$
\begin{aligned}
& d^{2} v_{L} / d u^{2}-(1 / u) d V_{L} / d u-\gamma^{2} V_{L}=0 \\
& d^{2} I_{L} / d u^{2}+(1 / u) d I_{L} / d u-\gamma^{2} I_{L}=0 \\
& d^{2} V_{L} / d u^{2}-(1 / u) d V_{L} / d u-\gamma^{2} V_{L}=0 \\
& d^{2} I_{L} / d u^{2}+(1 / u) d I_{L} / d u-\gamma^{2} I_{L}=0
\end{aligned}
$$

where

$$
\gamma \equiv \sqrt{z y}=\sqrt{z_{0} \bar{y}_{0}}
$$

Thus, (156) and (157) are seen to be identical in form to (158) and (159). Hence, the solutions are identical in form also. It is to be noted that $\gamma$ is independent of the taper of the line.

Equations (156) through (159) are forms of Bessel's equations and have the following solutions, respectively: *

$$
\begin{aligned}
& V_{L}=u\left[C_{1} J_{1}(G u)+C_{2} Y_{1}(G u)\right] \\
& I_{L}=\left[C_{3} J_{0}(G u)+C_{4} Y_{0}(G u)\right]
\end{aligned}
$$

where $J_{0}(G u)$ and $J_{1}(G u)$ are Bessel functions of the first kind, $Y_{0}(G u)$ and $\mathrm{Y}_{1}(\mathrm{Gu})$ are Bessel functions of the second kind, ${ }^{* * *} \mathrm{C}_{1}, \mathrm{C}_{2}, \mathrm{C}_{3}$, and $\mathrm{C}_{4}$ are integration constants, and

$$
G=\sqrt{-\gamma^{2}}
$$

* See, for example, Eugene Jahnke and Fritz Emde, "Tables of Functions with Formulae and Curves, "4th ed., New York: Dover Publications, 1945, p. 146, 7(1).

*** Also, called Neumann functions. 


$$
\mathrm{u}=\mathrm{k} \mp \mathrm{x}
$$

For the boundary conditions,

$$
\begin{aligned}
& \mathrm{x}=0 \\
& \mathrm{u}=\mathrm{k} \\
& \mathrm{V}_{\mathrm{L}}=\mathrm{V}_{\mathrm{R}} \\
& \mathrm{I}_{\mathrm{L}}=\mathrm{I}_{\mathrm{R}} \\
& \mathrm{Z}_{\mathrm{R}}=\mathrm{V}_{\mathrm{R}} / \mathrm{I}_{\mathrm{R}}
\end{aligned}
$$

the constants become

$$
\begin{aligned}
& C_{1}=(\pi G / 2)\left[V_{R} Y_{0}(G k) \pm\left(z_{0} I_{R} / G\right) Y_{1}(G k)\right] \\
& C_{2}=-(\pi G / 2)\left[V_{R} J_{0}(G k) \pm\left(z_{0} I_{R} / G\right) J_{1}(G k)\right] \\
& C_{3}= \pm\left(\pi y_{0} k / 2\right)\left[V_{R} Y_{0}(G k) \pm\left(z_{0} I_{R} / G\right) Y_{1}(G k)\right] \\
& C_{4}=\mp\left(\pi y_{0} k / 2\right)\left[V_{R} J_{0}(G k) \pm\left(z_{0} I_{R} / G\right) J_{1}(G k)\right] .
\end{aligned}
$$

Using (170) through (173) in (161) and (162), the general expressions for $V_{I}$ and $I_{L}$ result,

$$
\begin{aligned}
V_{L}=u(\pi G / 2) & \left\{\left[V_{R} Y_{0}(G k) \pm\left(z_{o} I_{R} / G\right) Y_{1}(G k)\right] J_{1}(G u)\right. \\
& \left.-\left[V_{R} J_{o}(G k) \pm\left(z_{o} I_{R} / G\right) J_{1}(G k)\right] Y_{1}(G u)\right\}
\end{aligned}
$$




$$
\begin{aligned}
I_{L}= \pm\left(\pi y_{0} k / 2\right)\{ & {\left[V_{R} Y_{0}(G k) \pm\left(z_{0} I_{R} / G\right) Y_{1}(G k)\right] J_{0}(G u) } \\
& \left.-\left[V_{R} J_{0}(G k) \pm\left(z_{0} I_{R} / G\right) J_{1}(G k)\right] Y_{0}(G u)\right\}
\end{aligned}
$$

and, of course,

$$
\mathrm{z}_{\mathrm{L}}=\mathrm{V}_{\mathrm{L}} / \mathrm{I}_{\mathrm{L}} \quad \text {. }
$$

At this point the expressions may be simplified for study by assuming a lossless line.

The Lossless Linear Line. - - The following discussion considers the line to be lossless for purposes of obtaining the current, voltage, and input impedance, that is;

$$
\begin{aligned}
& R_{0}=0 \\
& G_{0}=0 .
\end{aligned}
$$

Thus, using (177) and (178),

$$
\begin{aligned}
& z_{0}=j \omega L_{0} \equiv j x_{0} \\
& y_{0}=j \omega C_{0} \equiv j B_{0} \\
& \gamma=j \omega \sqrt{L_{0} C_{0}} \equiv j \beta_{0}=j \omega / v^{\prime} \\
& z_{o O}=\sqrt{L_{0} / C_{0}} \\
& v^{\prime}=\left(1 / \sqrt{L_{0} C_{0}}\right) \\
& G=\sqrt{-\gamma^{2}}=\beta_{0}
\end{aligned}
$$


and

$$
\begin{aligned}
& V_{L}=u\left(\pi \beta_{0} / 2\right)\left\{\left[V_{R} Y_{0}\left(\beta_{0} k\right) \pm\left(z_{O} I_{R} / \beta_{O}\right) Y_{1}\left(\beta_{0} k\right)\right] J_{1}\left(\beta_{0} u\right)\right. \\
& \left.-\left[V_{R} J_{0}\left(\beta_{0} k\right) \pm\left(z_{0} I_{R} / \beta_{0}\right) J_{1}\left(\beta_{0} k\right)\right] Y_{1}\left(\beta_{0} u\right)\right\} \\
& I_{L}= \pm\left(\pi y_{0} k / 2\right)\left\{\left[V_{R} Y_{0}\left(\beta_{0} k\right) \pm\left(z_{0} I_{R} / \beta_{O}\right) Y_{1}\left(\beta_{0} k\right)\right] J_{0}\left(\beta_{0} u\right)\right. \\
& \left.-\left[V_{R} J_{0}\left(\beta_{0} k\right) \pm\left(z_{0} I_{R} / \beta_{0}\right) J_{1}\left(\beta_{0} k\right)\right] Y_{0}\left(\beta_{o} u\right)\right\} \\
& Z_{L}=V_{L} / I_{L}
\end{aligned}
$$

Equations (185), (186), and (187) represent the general expressions for a lossless linear line terminated at the origin by an arbitrary impedance, $\mathrm{Z}_{\mathrm{R}}$.

Similar expressions for two particular terminations, the open and the shorted lines, respectively, are developed in the next section. The Open Line $\left(Z_{R}=\infty\right)$. - - For this case the basic equations (185), (186), and (187) become

$$
\begin{aligned}
& V_{L}=\left(\pi V_{R} / 2\right)\left(Z_{o L} / Z_{o o}\right)\left(\beta_{o} k\right)\left(Y_{o k} J_{1}-J_{o k} Y_{l}\right) \\
& I_{L}= \pm j\left(\pi V_{R} / 2\right)\left(\beta_{o} k / Z_{o o}\right)\left(Y_{o k} J_{o}-J_{o k} Y_{o}\right) \\
& Z_{L}=\mp j Z_{o L}\left[\frac{Y_{o k} J_{1}-J_{o k} Y_{1}}{Y_{o k} J_{o}-J_{o k} Y_{0}}\right],
\end{aligned}
$$

since

$$
\mathrm{Z}_{\mathrm{R}}=\infty \rightarrow \mathrm{I}_{\mathrm{R}}=0 \text {, }
$$




$$
\begin{aligned}
& \left.\beta_{o} u=1(x / k)\right] \beta_{o} k=\left(z_{o L} / Z_{o o}\right) \beta_{o} k, \\
& u / k=1-(x / k)=z_{o L} / z_{o o} \\
& u / k=1+(x / k)=z_{o L} / z_{o o} \\
& \beta_{o} / y_{o}=-j \sqrt{z_{o} y_{o}} / y_{o}=-j z_{o o},
\end{aligned}
$$

and

$$
\begin{array}{ll}
\mathrm{Y}_{\mathrm{o}}\left(\beta_{\mathrm{o}} \mathrm{k}\right) \equiv \mathrm{Y}_{\mathrm{ok}}, & \mathrm{J}_{\mathrm{o}}\left(\beta_{\mathrm{o}} \mathrm{k}\right) \equiv \mathrm{J}_{\mathrm{ok}}, \\
\mathrm{Y}_{1}\left(\beta_{\mathrm{o}} \mathrm{u}\right) \equiv \mathrm{Y}_{1}, & \mathrm{~J}_{1}\left(\beta_{\mathrm{o}} \mathrm{u}\right) \equiv \mathrm{J}_{1},
\end{array}
$$

'The power loss in length, $\mathrm{dx}$, assuming the line to have small. losses, is

$$
p \doteq\left(\left.\left|I_{L}\right|\right|^{2} / 2\right) \operatorname{Rdx}
$$

When the method previously used in Chapters II and III is employed, the power loss in length, $l$, is

$$
\mathrm{P} \doteq \mathrm{R} / 2 \int_{0}^{\ell}\left|\mathrm{I}_{\mathrm{L}}\right|^{2} \mathrm{dx}
$$

where $\left|I_{L}\right|{ }^{2}$ is obtained from (189). Since the author was unable to execute the general integration analytically, graphical methods were used for particular conditions. This topic will be discussed more fully later in this chapter.

Next, the resonant properties of the open line are investigated by obtaining the resonant lengths of line analogous to the quarter-wave and half-wave resonance conditions of the uniform line, that is, for

$$
Z_{L}=0
$$


and

$$
\mathrm{Z}_{\mathrm{L}}=\infty \quad,
$$

respectively. Thus, for odd-multiple quarter-wave resonances, the following equation results from (187):

$$
\mathrm{Y}_{\mathrm{ok}} \mathrm{J}_{1}-\mathrm{J}_{\mathrm{ok}} \mathrm{Y}_{1}=0
$$

The approximate solution of (202) may be obtained from the literature.* The solution will be of the form

$$
\begin{gathered}
\left(\beta_{\mathrm{o}} u\right)_{\mathrm{n}}=\frac{\mathrm{n}-0.5}{|1-(\mathrm{k} / \mathrm{u})|} \pi \mathrm{K}_{\mathrm{n}} \\
(\mathrm{n}=0,1,2,3, \ldots) .
\end{gathered}
$$

A discussion of $\mathrm{K}_{\mathrm{n}}$ is given later in this chapter. From (154) and (155), for $x$ equals $l$,

$$
\mathrm{u} \equiv \overline{\mathrm{u}}=\mathrm{k} \mp \ell \text {. }
$$

Then

$$
\mathrm{k} / \overline{\mathrm{u}}=\frac{1}{1 \mp(\mathrm{k} / \overline{\mathrm{u}})}=\mathrm{z}_{\mathrm{Oo}} / \mathrm{z}_{\mathrm{oS}},
$$

so that

$$
\left(\beta_{\mathrm{o}} \overline{\mathrm{u}}\right)_{\mathrm{n}}=\left(\beta_{\mathrm{o}}\right)_{\mathrm{n}} \overline{\mathrm{u}}=\frac{\mathrm{n}-0.5}{|1 \cdot(\mathrm{k} / \overline{\mathrm{u}})|} \pi \mathrm{K}_{\mathrm{n}} .
$$

*Jahnke and Emde, op. cit., p. 206-209. 
When both sides of (206) are multiplied by $\ell$ and divided by $\bar{u}$,

$$
\left(\beta_{\mathrm{o}}\right)_{\mathrm{n}} \ell=\ell \frac{\mathrm{n}-0.5}{|\overline{\mathrm{u}}-\mathrm{k}|} \pi \mathrm{K}_{\mathrm{n}} \text {, }
$$

but

$$
\overline{\mathrm{u}} \cdot \mathrm{k}=\mathrm{k} \mp \ell-\mathrm{k}=\mp \ell \text {. }
$$

So, equation (207) becomes

$$
\left(\beta_{0}\right)_{n} \ell=(n-0.5) \pi K_{n} .
$$

Now

$$
(n-0.5) \pi,
$$

for

$$
\mathrm{n}=1,2,3, \ldots
$$

is seen to be the successive odd-multiple quarter-wave resonant lengths in radians of the uniform line, that is,

$$
\pi / 2,3 \pi / 2,5 \pi / 2, \ldots
$$

Therefore, the quantity $K_{n}$ in (209) is recognized as the ratio of the quarter-wave resonant lengths (in radians) of the linear line to those of the uniform line. Thus, (209) may be written

$$
\frac{\left(\beta_{\mathrm{oL}}\right)_{\mathrm{n}} \ell}{\left(\beta_{\mathrm{o}}\right)_{\mathrm{n}} \ell_{\mathrm{o}}}=\mathrm{K}_{\mathrm{n}}
$$

where the subscript " $L$ " is introduced to indicate $\beta_{0}$ for the linear line. 
Actually, since the left hand side is a ratio of products, it may be rewritten as follows:

$$
\frac{\left(\beta_{\mathrm{oL}} \ell\right)_{\mathrm{n}}}{\left(\beta_{\mathrm{o}} \ell_{\mathrm{o}}\right)_{\mathrm{n}}}=\mathrm{K}_{\mathrm{n}}
$$

Hence, for a linear line and a uniform line operating at the same frequency $\left(f_{o L}=f_{0}\right)$

$$
\frac{(l)_{n}}{\left(l_{\mathrm{o}}\right)_{\mathrm{n}}} \equiv \frac{\ell_{\mathrm{n}}}{\ell_{\mathrm{on}}}=\mathrm{K}_{\mathrm{n}} \text {; }
$$

that is, the quantity $K_{n}$ is the ratio of the resonant lengths of the two lines. Similarly for two lines of the same length $(l=l$ o

$$
\frac{\left(\beta_{o L}\right)_{n}}{\left(\beta_{o}\right)_{n}}=\frac{\left(f_{o L}\right)_{n}}{\left(f_{o}\right)_{n}}=K_{n}
$$

which states that $K_{n}$ is the ratio of the resonant frequencies. As may be seen from the reference mentioned above, ${ }^{*} K_{n}$ is a function of the ratio

$$
\frac{\beta_{O L^{\bar{u}}}}{\beta_{O L^{k}}}=z_{O S} / Z_{O O}
$$

and has been plotted (for $n=1,2,3$ ) in Fig. 14 for both positive and negative lines. The ratio, $\mathrm{Z}_{\mathrm{oS}} / \mathrm{Z}_{\text {oo }}$ for the former, and $\mathrm{Z}_{\text {oo }} / \mathrm{Z}_{\mathrm{oS}}$ for the latter, is used for convenience. This allows the abscissa for each line to range from zero to one instead of from zero to infinity otherwise required.

Ibid., p. 207. 


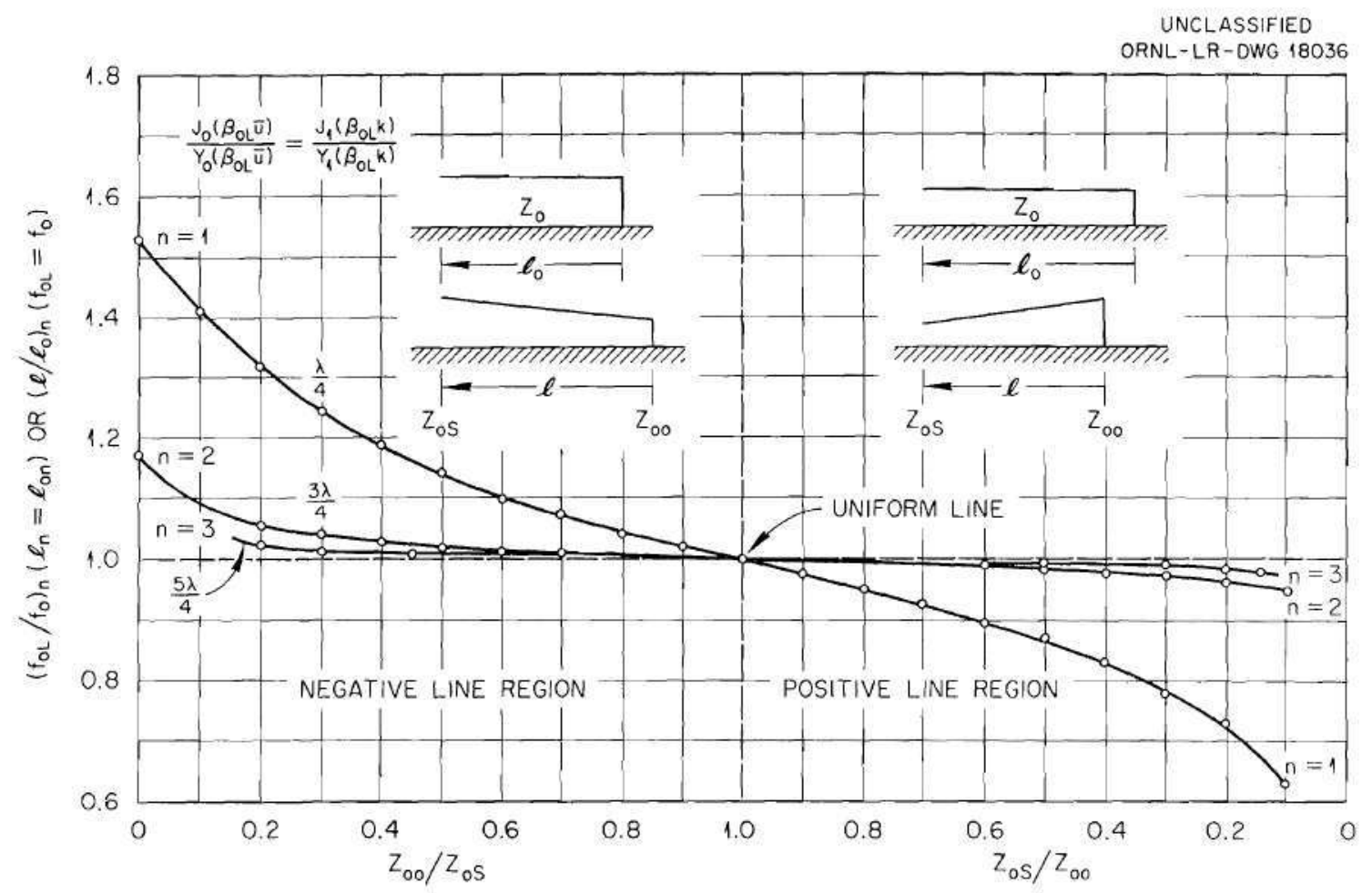

Fig. 14. Quarter-Wave Resonance vs Taper of the Lossless Linear Line. 
For half-wave resonance, the following condition results from (201) and (190):

$$
\mathrm{Y}_{\mathrm{ok}} \mathrm{J}_{\mathrm{o}}-\mathrm{J}_{\mathrm{ok}} \mathrm{Y}_{\mathrm{o}}=0
$$

The approximate solution of this equation is found in Jahnke and Emde on page 205, as a function of the ratios

$$
\frac{\beta_{O L^{k}}}{\beta_{O L^{\bar{u}}}} \text { or } \frac{\beta_{O L^{\bar{u}}}}{\beta_{O L^{k}}} \text {, }
$$

according to which is greater than unity. From (205) these ratios are either

$$
\mathrm{k} / \overline{\mathrm{u}}=\mathrm{z}_{\mathrm{Oo}} / \mathrm{z}_{\mathrm{oS}}
$$

or the reciprocal. The roots of (217) are given by

$$
\left[\left(Z_{O O} / Z_{O S}\right)-1\right]\left(\beta_{O L} \bar{u}\right)_{n}=a_{n}
$$

for the positive line, since

$$
\begin{gathered}
\frac{\beta_{O L}{ }^{k}}{\beta_{O L} \bar{u}}=k / \bar{u}=z_{O O} / z_{O S}>1 \\
{\left[\left(z_{O S} / z_{O O}\right)-1\right]\left(\beta_{O L}\right)_{n}=a_{n}}
\end{gathered}
$$

for the negative line. Equation (220) may be written

$$
\overline{\mathrm{u}}[(\mathrm{k} / \overline{\mathrm{u}})-1]\left(\beta_{\mathrm{oL}} l\right)_{\mathrm{n}}=\mathrm{a}_{\mathrm{n}} \ell .
$$


or

$$
\frac{\left(\beta_{o L} \ell\right)_{n}}{n \pi}=(1 / n \pi) \frac{a_{n} \ell}{k-(k-\ell)}=a_{n} / n \pi
$$

which is a function of $Z_{\text {oo }} / Z_{o S}$. Similarly, equation (222) may be written

$$
\mathrm{k}[(\overline{\mathrm{u}} / \mathrm{k})-1]\left(\beta_{\mathrm{oL}} \ell\right)_{\mathrm{n}}=\mathrm{a}_{\mathrm{n}} \ell,
$$

or

$$
\frac{\left(\beta_{o L} l\right)_{n}}{n \pi}=a_{n} / n \pi
$$

which is a function of $\mathrm{Z}_{\mathrm{oS}} / \mathrm{Z}_{00^{\circ}}$ Then (224) and (226) may be seen to represent the ratio of the electrical length of the linear line to that of the uniform line for integral-multiple half-wave resonances, since

$$
\left(\beta_{\mathrm{o}} \ell_{\mathrm{o}}\right)_{\mathrm{n}}=\mathrm{n} \pi
$$

for the uniform line. The equations (224) and (226) may be rewritten as follows:

$$
\frac{\left(\beta_{o L} l\right)_{n}}{\left(\beta_{0} l_{0}\right)_{n}}=a_{n} / n \pi
$$

so that for

$$
l=\ell_{0}
$$

a plot of $a_{n} / n \pi$ vs $Z_{o o} / Z_{o S}$ (or the reciprocal) represents the ratios of the half-wave resonant frequencies when the two lines are the same length. 
Similarly, for

$$
\beta_{\mathrm{OL}}=\beta_{\mathrm{O}},
$$

the same plot represents the ratio of the resonant lengths of the two lines. Fig. 15 is such a plot for both the positive and negative lines for $\mathrm{n}=1$, that is

$$
a_{1} / \pi \text { vs } z_{o o} / z_{o S}\left(\text { or } z_{o S} / z_{o o}\right) \text {. }
$$

The shorted line is considered in the next section.

The Shorted Line $\left(Z_{R}=0\right) \cdots$ For this case, equations $(185),(186)$, and (187) become

$$
\begin{aligned}
& V_{L}=\mp j(\pi / 2)\left(I_{R} Z_{o L}\right)\left(\beta_{o L} k\right)\left(Y_{1 k} J_{1}-J_{1 k} Y_{1}\right) \\
& I_{L}=(\pi / 2)\left(I_{R}\right)\left(\beta_{o L} k\right)\left(Y_{1 k} J_{0}-J_{1 k} Y_{0}\right) \\
& Z_{L}=\mp j Z_{o L}\left[\frac{Y_{1 k} J_{1}-J_{1 k} Y_{1}}{Y_{1 k} J_{0}-J_{1 k} Y_{0}}\right],
\end{aligned}
$$

since

$$
\begin{aligned}
& \mathrm{z}_{\mathrm{R}}=0 \rightarrow \mathrm{v}_{\mathrm{R}}=0 \\
& \mathrm{z}_{\mathrm{O}}=-\beta_{\mathrm{OL}}^{2} / \mathrm{y}_{\mathrm{O}}=-j \beta_{\mathrm{OL}} \mathrm{z}_{\mathrm{OO}} \\
& -\beta_{O L}^{2}=\mathrm{y}_{O} \mathrm{z}_{\mathrm{O}}
\end{aligned}
$$

and

$$
\mathrm{J}_{1}\left(\beta_{\mathrm{oL}} \mathrm{k}\right) \equiv \mathrm{J}_{1 \mathrm{k}} \quad, \quad \mathrm{Y}_{1}\left(\beta_{\mathrm{oL}} \mathrm{k}\right) \equiv \mathrm{Y}_{1 \mathrm{k}}
$$




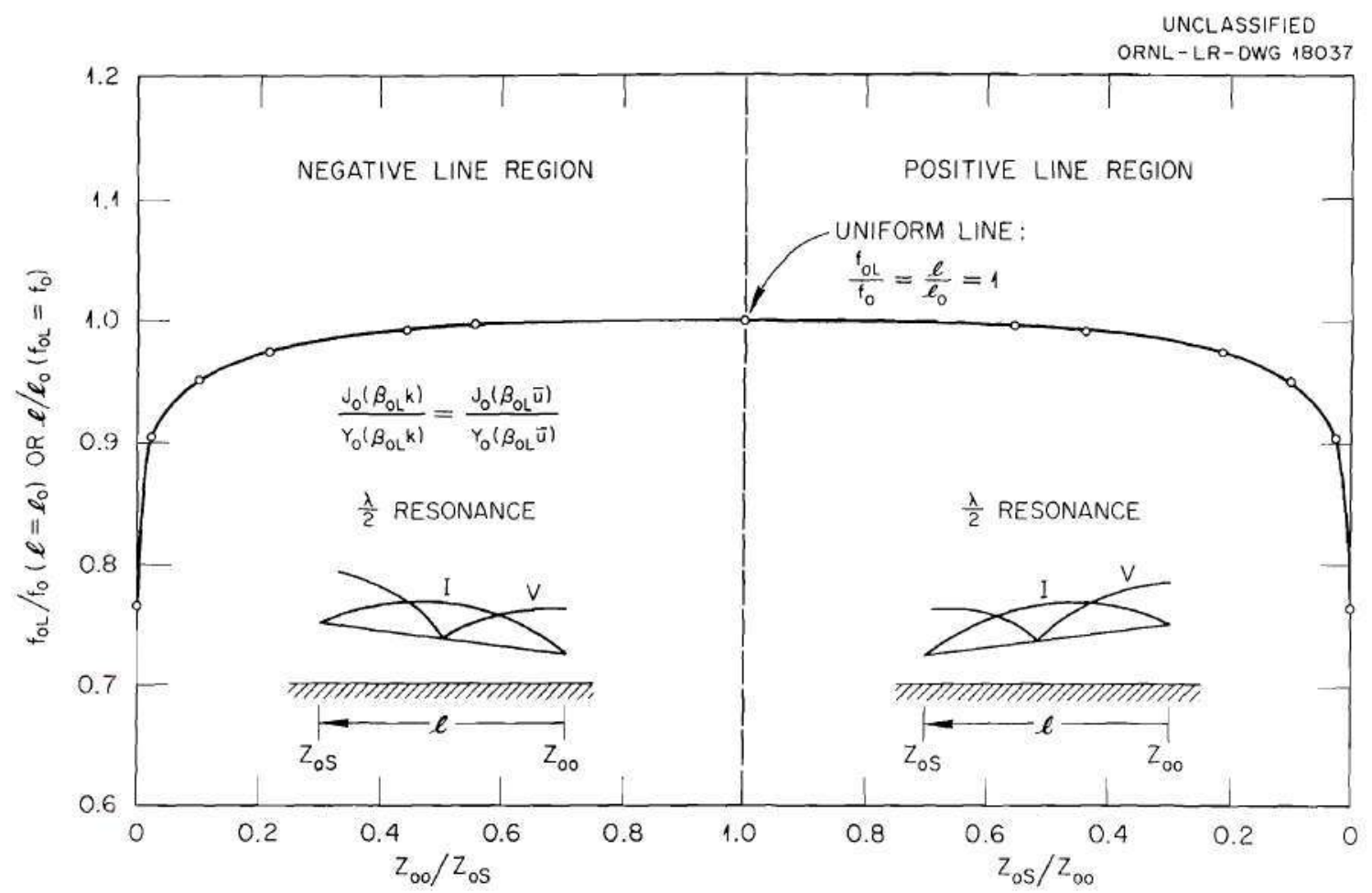

Fig. 15. Half-Wave Resonance vs Taper of the Open Lossless Linear Line. 


$$
\mathrm{J}_{1}\left(\beta_{\mathrm{oL}} \mathrm{u}\right) \equiv \mathrm{J}_{1} \quad, \quad \mathrm{Y}_{1}\left(\beta_{\mathrm{oL}} \mathrm{u}^{\mathrm{u}} \equiv \mathrm{Y}_{1} \quad .\right.
$$

The power loss in length, $l$, is:

$$
P_{L} \doteq(R / 2) \int_{0}^{l}\left|I_{L}\right|^{2} d x
$$

This expression is identical in form to (199) and was obtained in the same manner. The details of the graphical solution are taken up later in this chapter.

The quarter-wave and half-wave resonant properties of the shorted line are obtained from the condition

$$
Z_{L}=\infty
$$

and

$$
Z_{L}=0,
$$

respectively. Thus, the following equation is obtained from (234) for quarter-wave resonance:

$$
\mathrm{Y}_{1 \mathrm{k}} \mathrm{J}_{\mathrm{o}}-\mathrm{J}_{1 \mathrm{k}} \mathrm{Y}_{\mathrm{o}}=0
$$

This is seen to be identical in form to equation (202) with the arguments of $\mathrm{J}_{0}$ and $\mathrm{Y}_{0}$ interchanged with those of $\mathrm{J}_{1}$ and $\mathrm{Y}_{1}$. Therefore, the solution for this case will be of the form

$$
\begin{aligned}
& \left(\beta_{o L} \bar{u}\right)_{n}=\left[\frac{n-0.5}{|(k / \bar{u})-1|}\right] \pi K_{n} \\
& \left(\beta_{O L}\right)_{n} \ell=\left[\frac{n-0.5}{|k-\bar{u}|}\right] \pi K_{n},
\end{aligned}
$$


or

$$
K_{n}=\frac{\left(\beta_{o L} l\right)_{n}}{(n-0.5) \pi}
$$

which, of course, is the same result obtained in (209). Hence, the quan tity $\mathrm{K}_{\mathrm{n}}$ is either the ratio of the quarter-wave resonant frequencies for a linear line and a uniform line of the same length, or the ratio of the quarter-wave resonant lengths of a linear line and a uniform line operating at the same frequency.

Similarly, for half-wave resonances, from (242) and (234)

$$
\mathrm{Y}_{1 \mathrm{k}} \mathrm{J}_{1}-\mathrm{J}_{1 \mathrm{k}} \mathrm{Y}_{1}=0
$$

Several of the roots of this equation are tabulated in Jahnke and Emde as a function of $\mathrm{k} / \overline{\mathrm{u}}$ and $\overline{\mathrm{u}} / \mathrm{k}$ for the positive and negative line, respectively. The roots are given by

$$
\left[\left(Z_{o S} / Z_{o o}\right)-1\right]\left(\beta_{o L} \bar{u}\right)_{n}=A_{n}
$$

for the positive line, and

$$
\left[\left(z_{o S} / z_{o o}\right)-1\right]\left(\beta_{o L} k\right)_{n}=A_{n}
$$

for the negative line. Equation (248) may be rearranged as follows:

$$
\left[\left(Z_{o S} / Z_{o o}\right)-1\right]\left(\beta_{o L} \bar{u}\right)_{n}=[(k / \bar{u})-1]\left(\beta_{o L} l\right)_{n} \bar{u}=A_{n} l,
$$

or

$$
\frac{\left(\beta_{\mathrm{OL}} l\right)_{\mathrm{n}}}{\mathrm{n} \pi}=\mathrm{A}_{\mathrm{n}} / \mathrm{n} \pi .
$$


Similarly, for (249)

$$
\frac{\left(\beta_{\mathrm{oL}} l\right)_{\mathrm{n}}}{\mathrm{n} \pi}=\mathrm{A}_{\mathrm{n}} / \mathrm{n} \pi
$$

Thus, (251) and (252) are seen to be of the same form as (224) and (226). While they have analogous meaning to (224) and (226),

$$
a_{n} \neq A_{n} \text {. }
$$

Hence, (251) and (252) are plotted in Fig. 16 for $\mathrm{n}=1$.

The mathematical development of the linear line is concluded with equation (253). Illustrations of some of the above results are presented and discussed in the next several sections in an attempt to provide a more complete picture of the physical properties of the linear line.

Input Impedance of the Shorted Line vs Length. -- Equation (234) represents the input impedance of the shorted line. Rearranging the equation by normalizing with respect to the nominal characteristic impedance at the open end, the following equation results:

$$
Z_{N} \equiv Z_{L} / Z_{o S}=\mp j\left(Z_{o L} / Z_{o S}\right)\left[\frac{Y_{1 k} J_{1}-J_{1 k} Y_{1}}{Y_{1 k} J_{0}-J_{1 k} Y_{0}}\right] \text {. }
$$

With the frequency held constant, $\mathrm{Z}_{\mathrm{N}}$ is plotted in Fig. 17 as a function of the normalized length, $\mathrm{x} / \ell$, for two values of $\mathrm{Z}_{\mathrm{oS}} / \mathrm{Z}_{00^{\circ}}$ Input Impedance of the Shorted Line vs Frequency.--Fig. 18 is a sketch of the input impedance of a fixed length of shorted line as a function of frequency for two values of $\mathrm{Z}_{\mathrm{oS}} / \mathrm{Z}_{\mathrm{oo}^{\prime}}$ viz, 0.138 and 7.250 which correspond to values of taper constant of 18.56 and 2.56, respectively. Reference curves for the uniform line (with characteristic impedance values equal to the two end values of nominal characteristic impedance for the linear line) are included. Thus, Fig. 18 serves to show the trend of the impedance with taper constant and to show the difference in the general shape of the curves compared to the uniform line. 


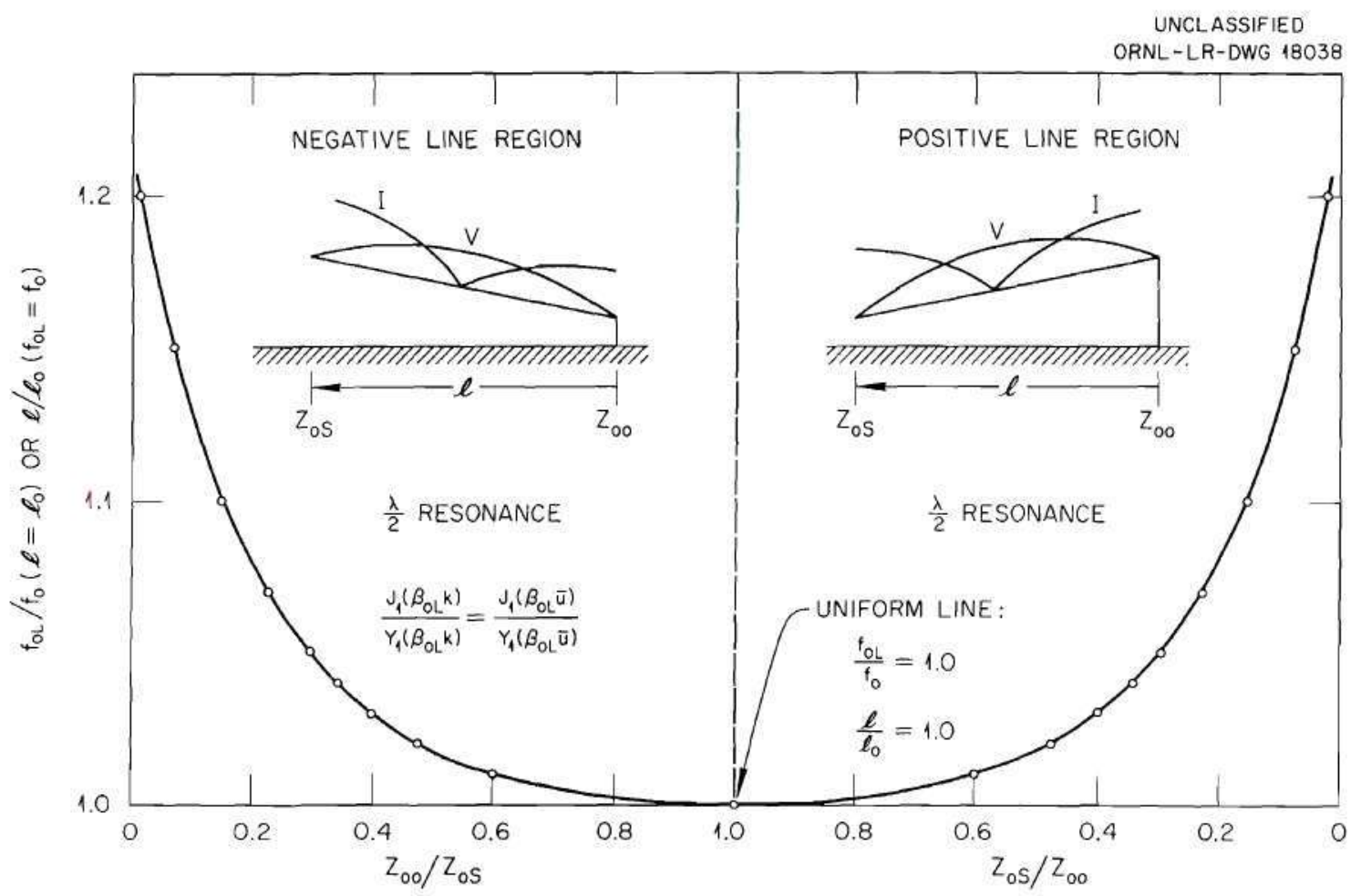

Fig. 16. Half-Wave Resonance vs Taper of the Shorted Lossless Linear Line. 
$-66-$

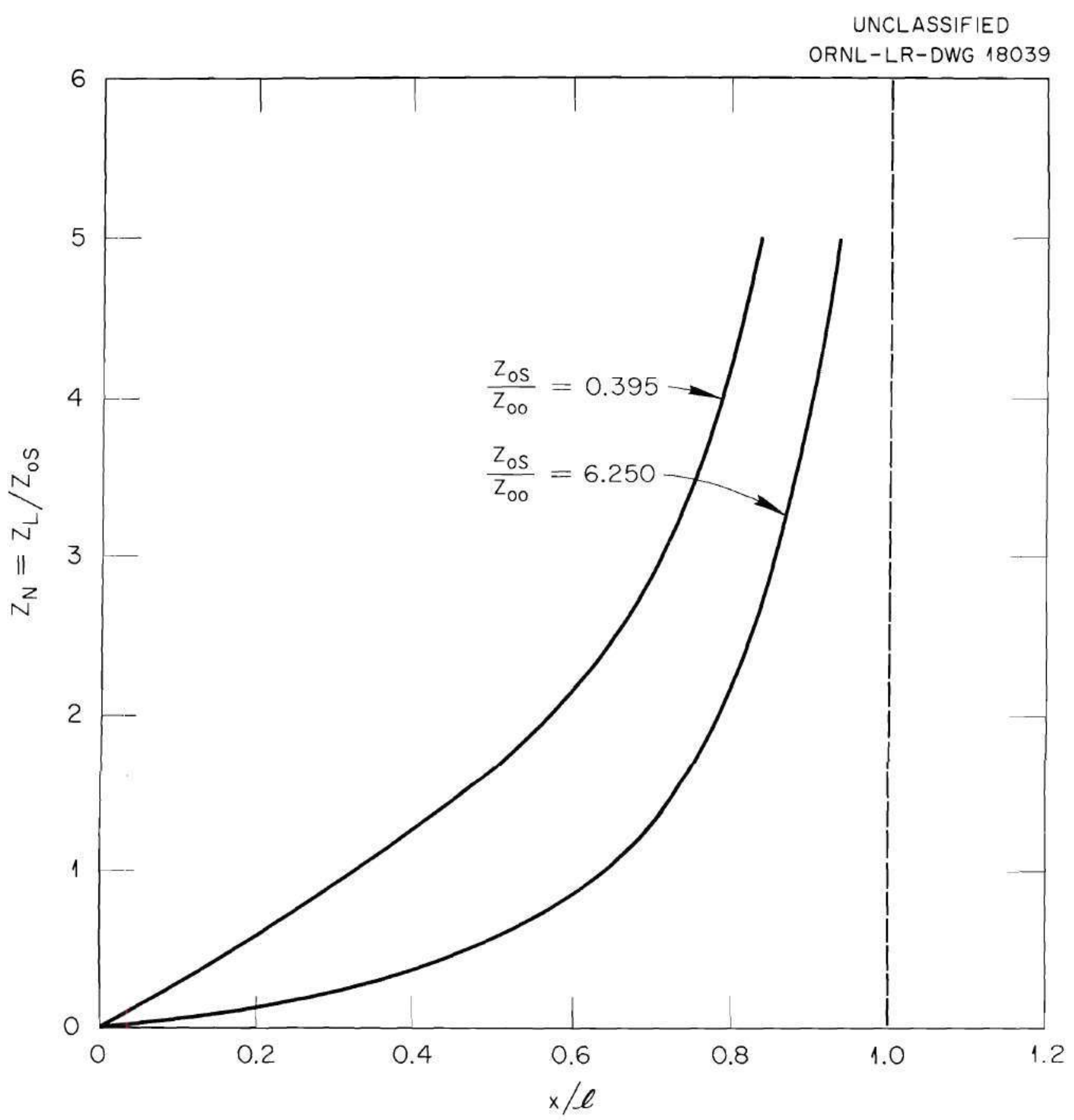

Fig. 17. A Plot of the Normalized Input Impedance vs Normalized Length of the Shorted Linear Line. 


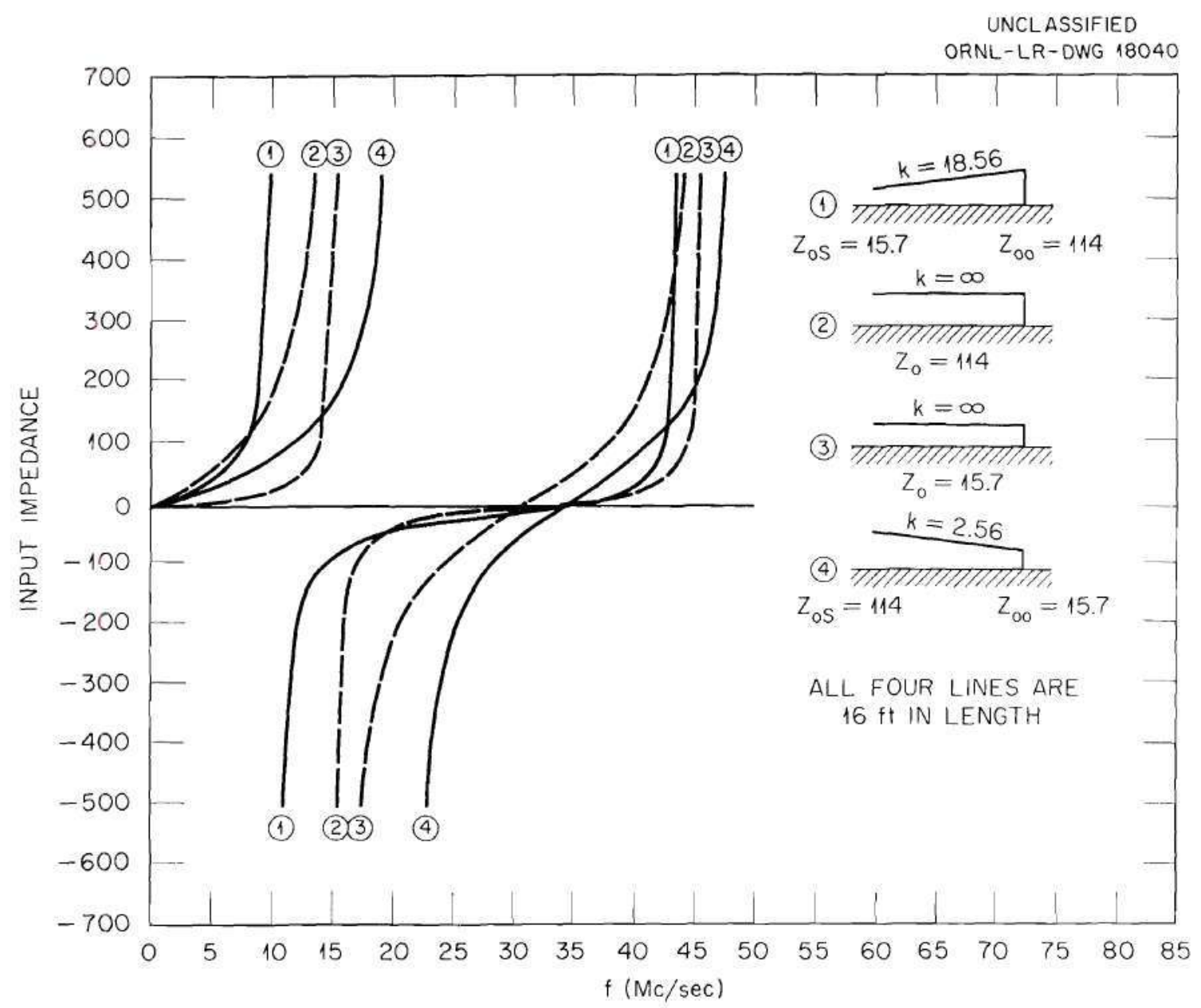

Fig. 18. Input Impedance vs Frequency for Two Linear and Two Uniform Lossless Lines. 
Voltage and Current Standing Waves on the Shorted Line.--To illustrate the difference in the general shape of the standing waves on a linear line to those on a similar lossless uniform line, impedance ratio contours of voltage and current are plotted in Fig. 19 and 20 from equations (232) and (233) after normalization as described below. First, from (232) for quarter-wave resonance

$$
\mathrm{x}=\ell
$$

and

$$
V_{L S}=\mp j(\pi / 2)\left(I_{R} Z_{o L}\right)\left(\beta_{o L} k\right)\left[Y_{1 k} J_{1}\left(\beta_{o L} \bar{u}\right)-J_{1 k} Y_{1}\left(\beta_{o L} \bar{u}\right)\right]
$$

where

$$
\overline{\mathrm{u}}=\mathrm{k} \mp \ell=\mathrm{kZ} \mathrm{oS}_{\mathrm{oo}} / \mathrm{Z}_{\mathrm{o}}
$$

$\mathrm{V}_{\text {LS }}$ is the voltage at the open end of the shorted line. When the value of $I_{R}$ from (256) is substituted in (232) and (233), the two equations become (after some algebraic manipulation)

$$
v_{L}=v_{L S}\left\{\frac{z_{o o}}{Z_{o S}}-\frac{x}{\ell}\left[\frac{z_{o o}}{Z_{o S}}-1\right]\right\}\left\{\frac{Y_{1 k} J_{1}-J_{1 k} Y_{1}}{Y_{1 k} J_{1}\left(\beta_{o L} L^{\bar{u}}-J_{1 k} Y_{1}\left(\beta_{O L} L^{\bar{u}}\right)\right.}\right\}
$$

and

$$
I_{L}= \pm j\left(V_{L S} / Z_{o S}\right)\left\{\frac{Y_{1 k} J_{o}-J_{1 k} Y_{o}}{Y_{1 k} J_{1}\left(\beta_{o L} \bar{u}\right)-J_{1 k} Y_{1}\left(\beta_{o L} \bar{u}\right)}\right\}
$$

Then the normalized values are defined as

$$
\mathrm{V}_{\mathrm{N}} \equiv \mathrm{V}_{\mathrm{L}} / \mathrm{V}_{\mathrm{LS}}
$$




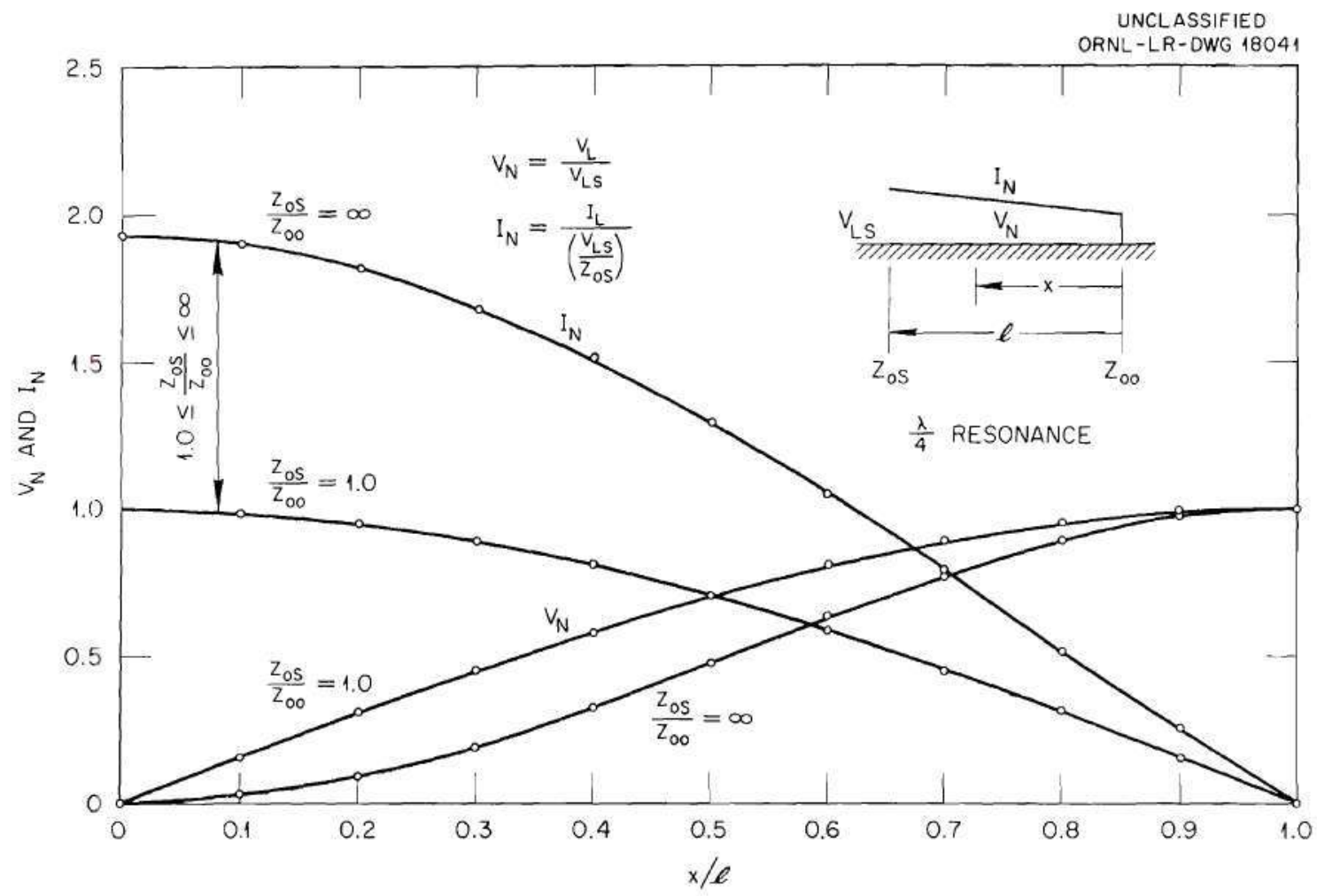

Fig. 19. Normalized Voltage and Current Standing Waves on the Shorted Linear Line. 


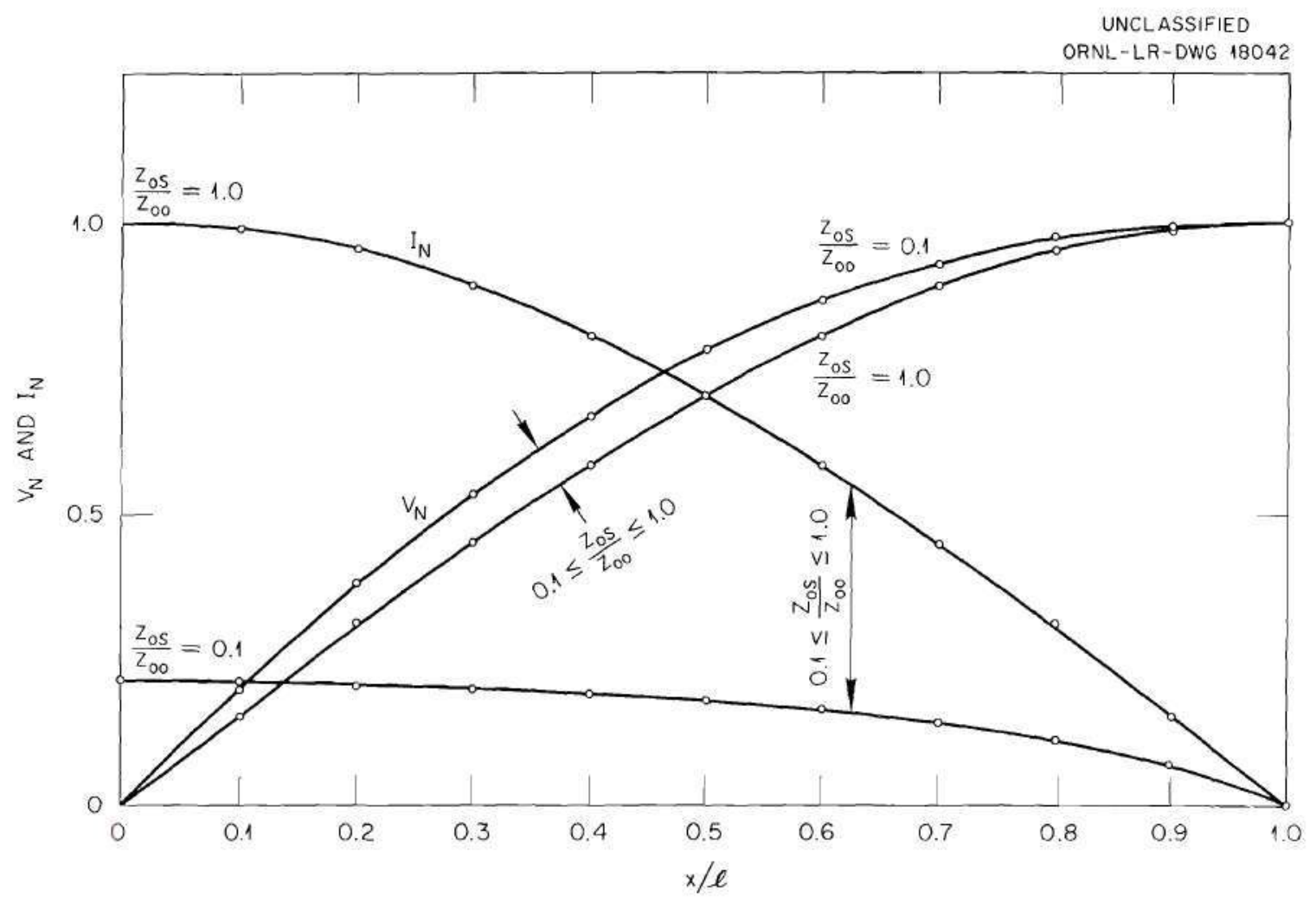

Fig. 20. Normalized Voltage and Current Standing Waves on the Shorted Linear Line. 


$$
I_{N}=\frac{\left|I_{L}\right|}{V_{L S} / Z_{O S}}
$$

These expressions may be obtained as a function of the normalized length, $x / l$, for chosen values of $Z_{o o} / Z_{o S}$ This is true because, for a fixed frequency, (260) and (261) are functions of $Z_{o o} / Z_{o S}$ from

$$
\begin{aligned}
& \beta_{O L} \bar{u}=(\pi / 2) \frac{K_{1}}{\left(Z_{O S} / Z_{o O}\right)-1} \\
& \beta_{O L} k=(\pi / 2) \frac{K_{1}}{\left[-\left(Z_{O S} / Z_{o O}\right)\right]} \\
& \beta_{O L}{ }^{u}=(\pi / 2) K_{1}\left[\frac{1}{\left[1-\left(Z_{O S} / Z_{O O}\right]\right.}-(x / \ell)\right]
\end{aligned}
$$

for the positive line, and similarly, from

$$
\begin{aligned}
& \beta_{O L^{\bar{u}},} \\
& \beta_{O L^{k}},
\end{aligned}
$$

and

$$
\beta_{\mathrm{OL}} \mathrm{u}^{\mathrm{u}}
$$

(which are functions of $\mathrm{Z}_{\mathrm{oS}} / \mathrm{Z}_{\mathrm{oo}}$ ) for the negative line.

Power Loss of the Quarter-Wave Resonant Line $\left(Z_{R}=0\right) .--$ The power loss of a shorted line operating in quarter-wave resonance may be obtained by graphical integration of equation (240) as previously noted. This equation may be expressed in the following form for the positive line: 


$$
\begin{aligned}
P_{O L} & =\left(R / 2 \beta_{o L}\right)\left(V_{L S} / Z_{O S}\right)^{2} \frac{\int_{\bar{\psi}}^{\psi}\left[Y_{1 k} J_{o}-J_{1 k} Y_{o}\right]^{2} d\left(\beta_{o L} u\right)}{\left[Y_{1 k} J_{1}\left(\beta_{O L} \bar{u}\right)-J_{1 k} Y_{1}\left(\beta_{O L} \bar{u}\right)\right]^{2}} \\
& \equiv\left(R / 2 \beta_{O L}\right)\left(V_{L S} / Z_{O S}\right)^{2} \bar{K}
\end{aligned}
$$

where

$$
\begin{aligned}
& \psi=\beta_{O L}{ }^{k} \\
& \bar{\psi}=\beta_{O L} \bar{u} .
\end{aligned}
$$

Then the ratio of (268) to equation (49) from Chapter II is

$$
P_{O L} / P_{0}=\left[\frac{\left(R / 2 \beta_{O L}\right)\left(V_{L S} / Z_{O S}\right)^{2} \bar{K}}{\left(r / 2 \beta_{0}\right)\left(V_{S} / Z_{O}\right)^{2}(\pi / 4)}\right]
$$

which is the ratio of power loss of the linear line to that of the uniform line. This ratio becomes

$$
P_{O L} / P_{O}=(R / r)\left(\beta_{0} / \beta_{O L}\right)\left(V_{L S} / V_{S}\right)^{2}\left(Z_{O} / Z_{O S}\right)^{2}(4 / \pi) \bar{K}
$$

For the negative line, the ratio is given by the same expression with the limits of integration reversed. Now, if

$$
R / r=V_{L S} / V_{S}=Z_{0} / Z_{O S}=\beta_{O} / \beta_{O L}=1
$$

then the ratio becomes

$$
P_{O L} / P_{O}=(4 / \pi) \frac{\int_{\bar{\psi}}^{\psi}\left[Y_{1 k} J_{0}-J_{1 k} Y_{0}\right]^{2} d\left(\beta_{o L} u\right)}{\left[Y_{1 k} J_{1}\left(\beta_{o L} \bar{u}\right)-J_{1 k} Y_{1}\left(\beta_{O L} \bar{u}\right)\right]^{2}} .
$$


The composite curve for both the positive and negative lines is plotted from (272) as a function of $\mathrm{Z}_{\mathrm{oS}} / \mathrm{Z}_{\text {oo }}$ for the positive line, and $\mathrm{Z}_{\mathrm{oo}} / \mathrm{Z}_{\mathrm{oS}}$ for the negative line, in Fig. 21.

The development and illustration of the theoretical results are concluded with this section.

Summary. A mathematical treatment paralleling the method used in Chapter III is employed in this chapter to develop the general equations which describe the linear line. These equations, (174), (175), and (176), are in terms of Bessel functions of the first kind of orders zero and one and Neumann functions of orders zero and one.

Emphasis is placed upon the lossless and low-los lines since the interest is in resonant applications. Similar to the development in Chapter III, the theory for both the open and shorted lossless lines is given. In addition, the characteristics of the shorted line are illus trated from theory with a variety of graphs. These graphs include quarter-wave and half-wave resonance vs taper, input impedance vs normalized length, input impedance vs frequency, voltage impedance vs standing waves vs distance, and power loss vs taper. A detailed dis. cussion of the linear line is deferred to Chapter VI.

The results in this chapter are limited to transmission lines whose change in spacing with respect to length along the line is small. These limitations are considered in Chapter VI.

Experimental verification of the theory is presented in the next chapter. 


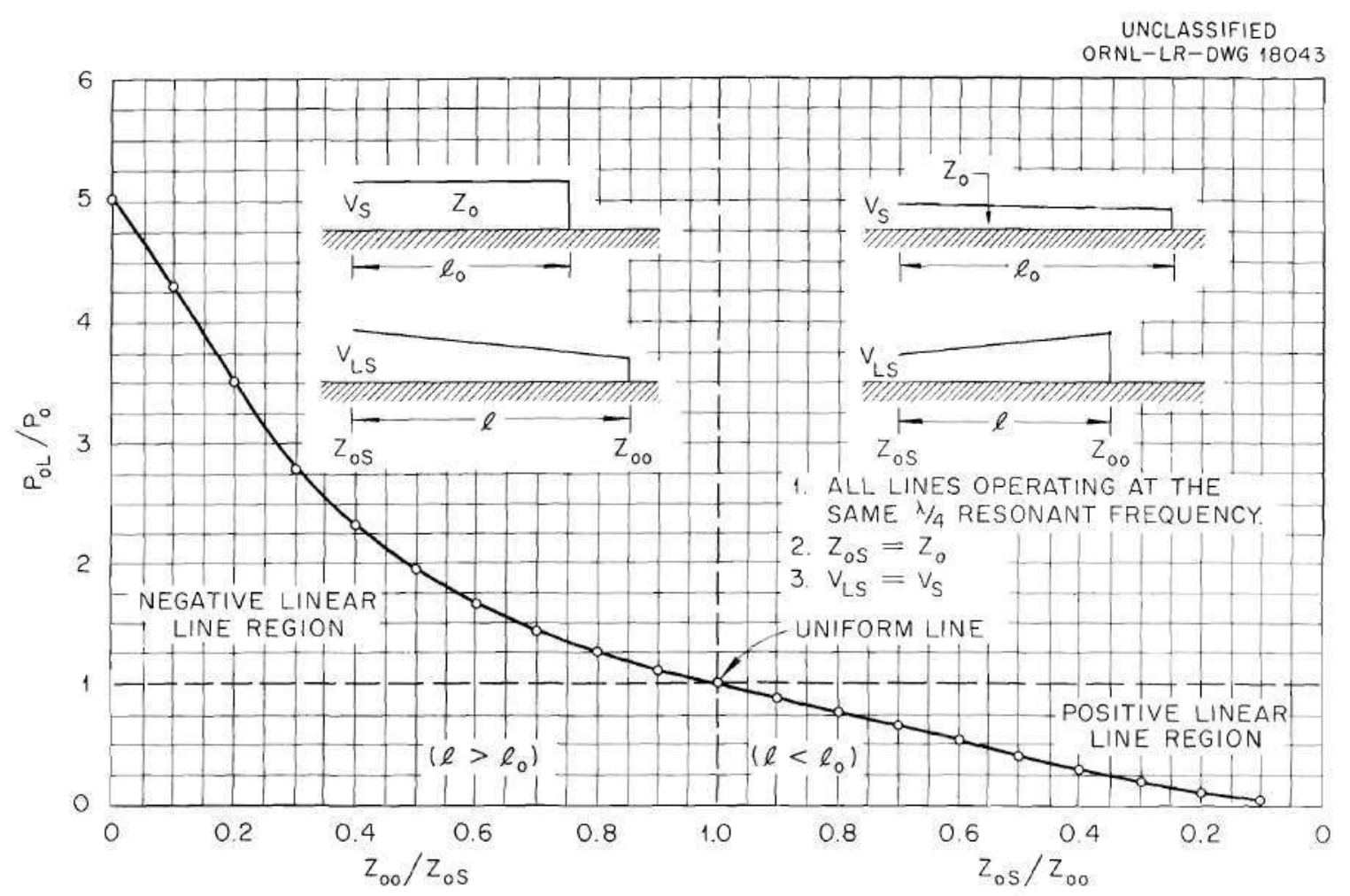

Fig. 21. Power Loss Ratio vs. Taper of the Quarter-Wave Linear Line. 


\section{CHAPTER V}

\section{EXPERIMENTAL RESULTS}

Introduction. --To verify the theory in Chapter IV, five linear lines were constructed. Two problems arise when the correlation between theoretical and experimental results is to be determined. One, the theory neglects transmission line "end effects". Two, the experimental results are subject to inherent measurement errors. Both of these difficulties tend to confuse the observed agreement between theory and practice.

In order to circumvent these difficulties in part, it was decided to construct four uniform lines very similar to four of the linear lines mentioned above. Since the intrinsic agreement between theoretical and experimental data for the uniform line is known to be quite precise, discrepancies between measured and theoretical results for the four uniform lines could be ascribed to measurement, geometrical, and end effect errors. Thus, from this procedure, two pieces of information resulted. One, data from the uniform lines indicated the correlation to expect assuming complete agreement between theoretical and experimental data for the linear lines. Two, the correlation between the measured and theoretical data for the uniform lines yielded information on the accuracy of the measurement technique.

Although the interest was not in precise absolute data, considerable care was exercised in the design of the experimental lines and in the choice of measurement apparatus to obtain accurate results. Description of the Experimental Lines. - - The four uniform lines mentioned above were obtained from the apparatus shown in Fig. 22. Each of these lines consisted of a sheet of copper foil 0.005 inch thick which was mounted parallel to a $r-f$ ground plane. Such a system was obtained by covering the underside of a plywood sheet with foil and supporting it above the surface of a similarly covered table top. At the radio 


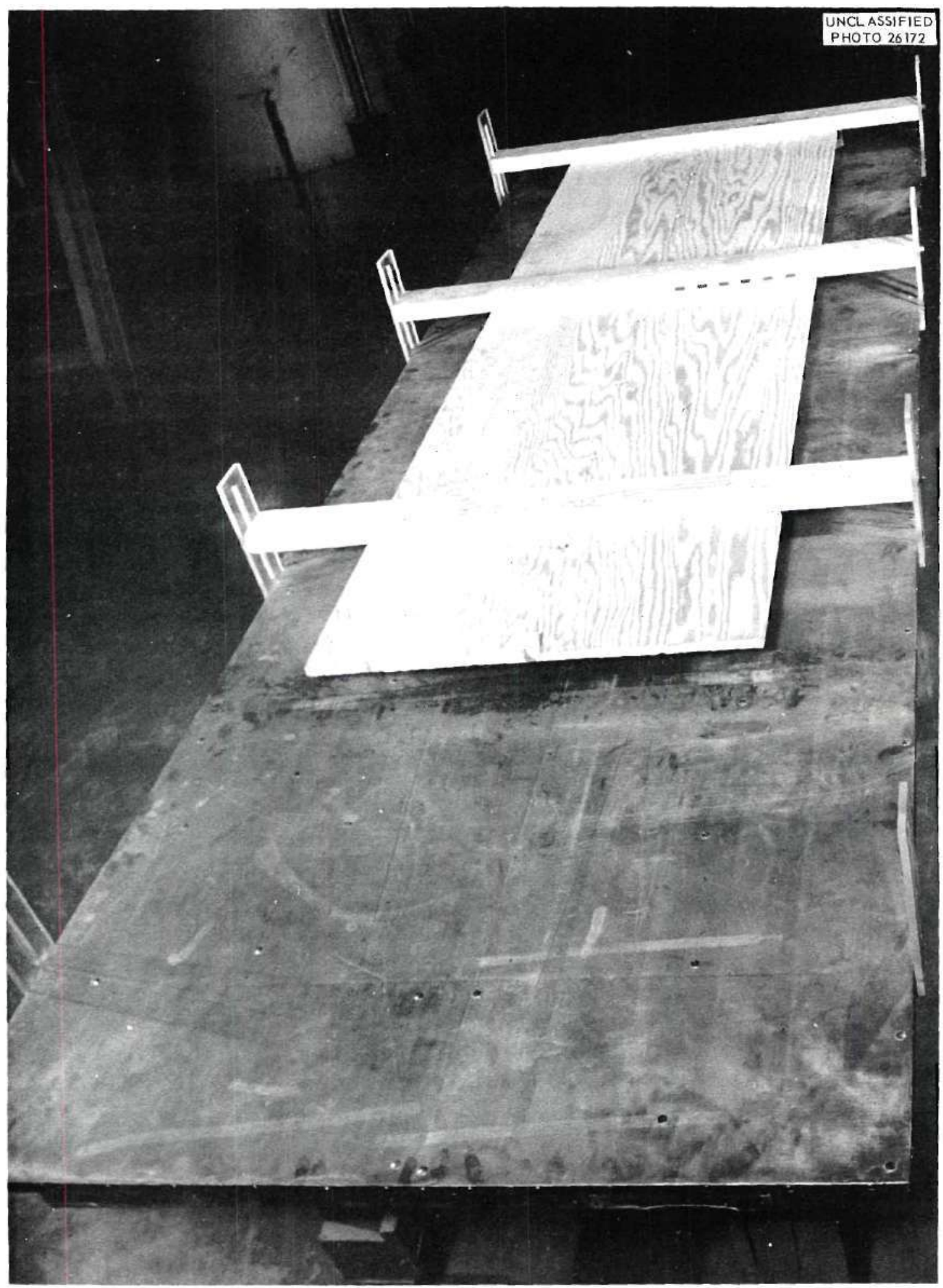

Fig. 22. Experimental Uniform Strip Line. 
frequencies involved in the measurements, 3 to $31 \mathrm{Mc} / \mathrm{sec}$, the $\mathrm{r}-\mathrm{f}$ current traveled essentially on the surface of the copper so that a thickness of 0.005 inch was sufficient to confine the electromagnetic field between the parallel surfaces. Some fringing existed, of course, but the spacing of the conductor above the ground plane was kept small compared to the width of the conductor to reduce this effect. The foil was 30 inches wide and 8 feet in length, whereas, the ground plane was 48 inches wide to insure that the fringe field was confined to the table top.

By the image principle, the geometry described above yielded a type of line known as a "strip line" which had a characteristic imped-ance that was a direct function of the spacing between the ground plane and the conductor (strip). Specifically,

$$
\begin{aligned}
& Z_{0}=377 \mathrm{~d} / \mathrm{w} \\
& (\mathrm{w}>>\mathrm{d})
\end{aligned}
$$

where $\underline{w}$ is the width of the strip (30 inches) and $\underline{d}$ is the spacing mentioned.

Due to the condition on (273), spacings of the four uniform strip lines constructed were 1/2, 1, 1 1/2, and 2 inches, respectively. Thus, d/w ranged from $1 / 60$ to $1 / 15$. The smallest spacing ( $1 / 2$ inch) was chosen as a compromise between an effort to minimize end effects and an effort to avoid significant errors in spacing. (End effect errors approach zero as the spacing approaches zero, whereas, errors in spacing approach zero as the spacing approaches very large values.)

The accuracy of the spacing of the strip above the ground plane was estimated to be better than 3 per cent at the nominal value of $1 / 2$ inch and proportionately better at the other three values. This was ac complished by establishing the desired nominal spacing with accurately dimensioned $1 / 2$ inch thick strips of lucite placed at intervals of one foot along, and transverse to, the length of plywood sheet. As shown in Fig. 23, the plywood was stiffened and ultimately supported by three 


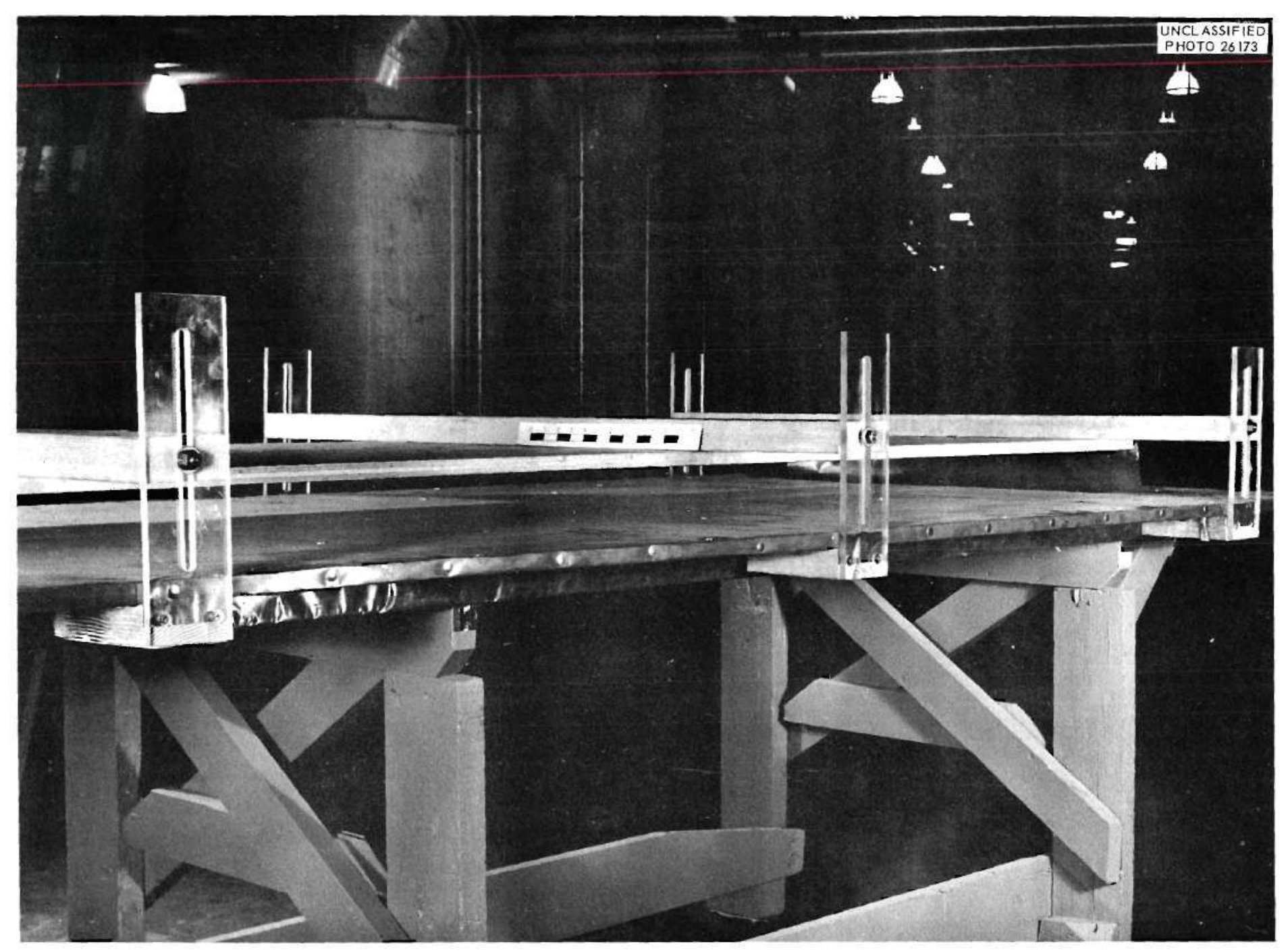

Fig. 23. Method of Support for the Experimental Uniform Strip Line. 
two-by-fours. Studs attached to the ends of the two-by-fours provided a means of clamping the plywood to slotted lucite supports which were attached to the edges of the table. Thus, with the clamps initially loosened, the plywood was allowed to rest on the accurately dimensioned lucite strips. The clamps were then tightened. Finally, by carefully checking each strip and adjusting the plywood sheet until each spacer barely touched the strip of foil as the spacer was removed, the line was accurately spaced to an estimated 0.010 inch.

It is to be noted that the strip line was chosen because of the linear dependence of $Z_{0}$ on the spacing which permitted simple fabrication and easy conversion to different lines. Four linear lines were obtained from this apparatus merely by inclining the plywood with respect to the ground plane, a particularly convenient technique. Accurate spacings were obtained by the method described in the previous paraw graph. The spacings at the open end of three of the linear lines were $1 / 2$ inch, while the spacings at the shorted end were $1,11 / 2$, and 2 inches, respectively. The fourth line was spaced 1 inch at the open end and 2 inches at the shorted end. One of these linear lines is shown. in Fig. 24 .

In addition to the lines described above, three open-wire lines, one of each type, were constructed. Fig. 25 shows the exponential line. Each of the three lines was 10 feet in length and was constructed from 0.102 inch diameter copper wire spaced 12.75 inches above the ground plane. The spacing between conductors at the corresponding ends of the exponential and linear lines was the same, namely, 4.95 inches and 0.255 inch. The spacing of the uniform line was 0.255 inch. These three lines were constructed for comparison with the strip lines described above. The input impedance was obtained for the uniform and linear lines but, due to the limitations of the measurement apparatus * above the critical frequency $(12.1 \mathrm{Mc} / \mathrm{sec})$, the input impedance of the exponential line could not be obtained.

\footnotetext{
* To be described subsequently。
} 


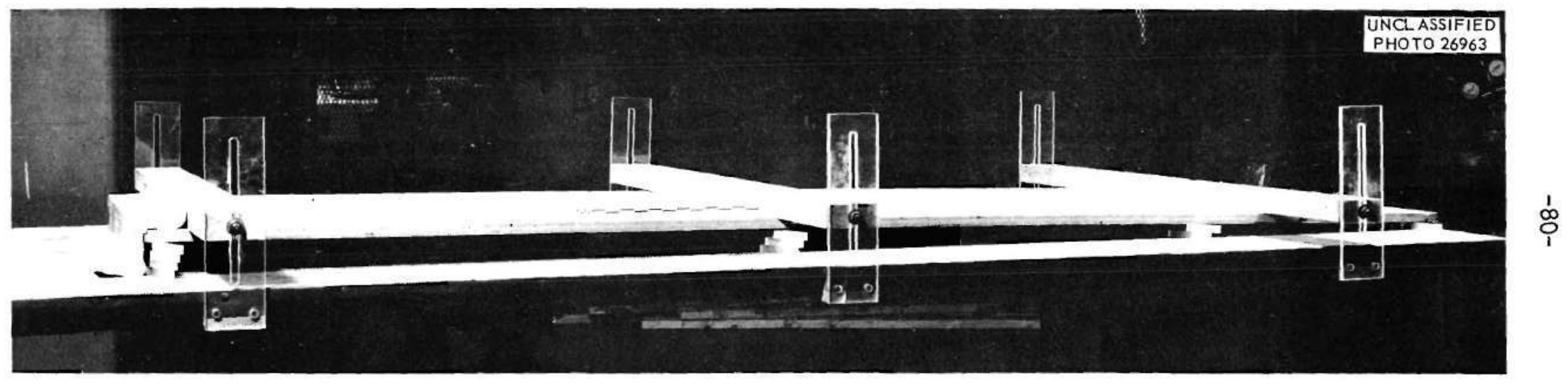

Fig. 24. Experimental Linear Strip Line. 


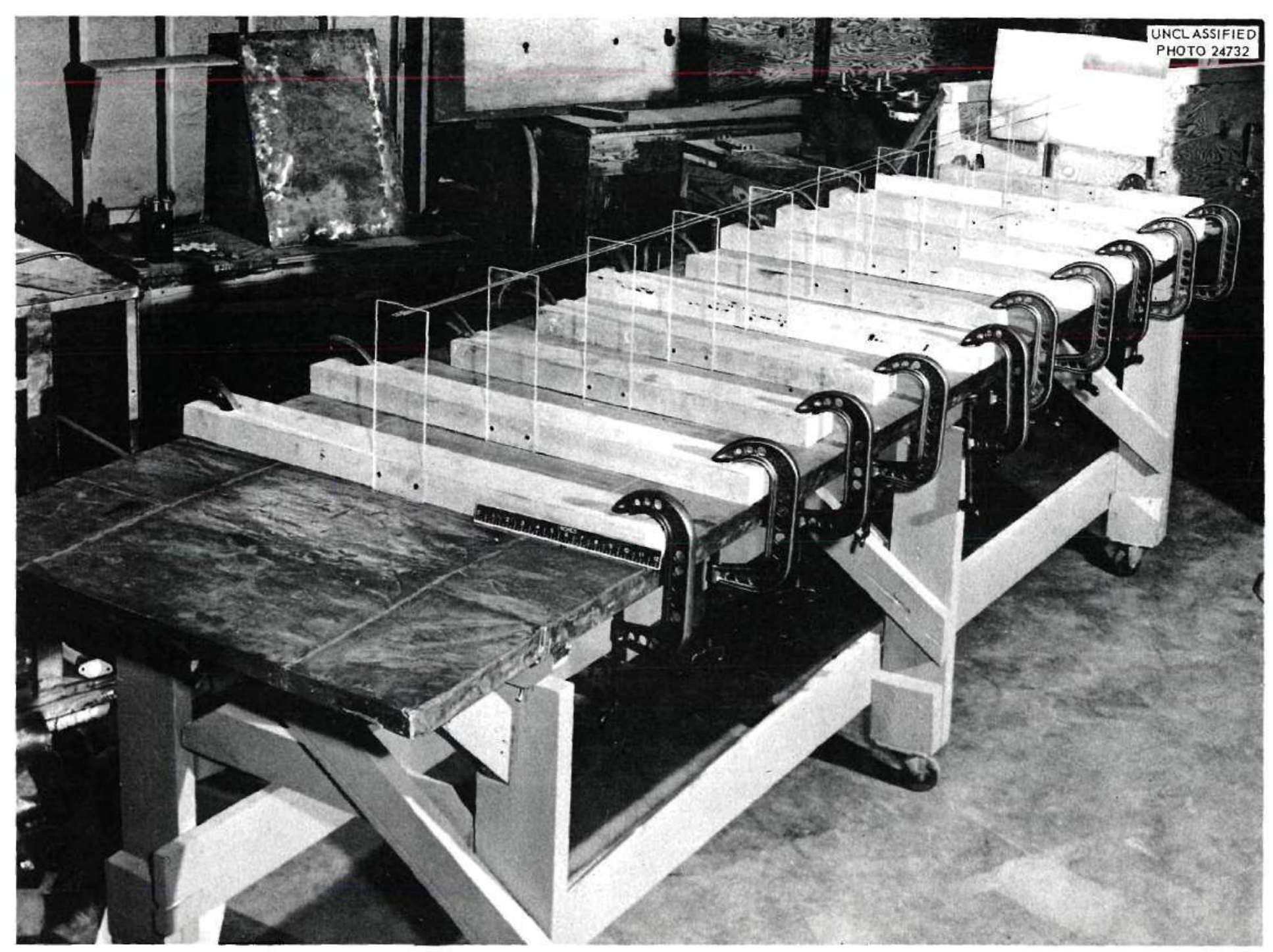

Fig. 25. Experimental Exponential Open Wire Line. 
Description of the Measuring Equipment.- The two characteristics of the linear line considered sufficient to show the correlation between theory and practice were the resonant frequency and the input impedance vs frequency of the shorted line. These characteristics were chosen because they were the two most readily obtained with the highest accuracy. The properties of the shorted line were chosen for two reasons. One, the end effects of the line (which was neglected in the theory) could be reduced to some extent at the shorted end. Two, the shorted line permitted input impedance (reactance) measurements of low-loss lines up to quarter wave resonance with a precision capacitor, as well as with an impedance bridge. The capacitance method permitted measurements on the open - wire lines which did not have a grounded terminal and, therefore, were not easily measured with a bridge.

Frequency, impedance, and length were the primary quantities measured. A Collins Radio Communications Receiver Model $51 \mathrm{~J}-3$ was used to measure frequency. This instrument covers the range from $540 \mathrm{Kc} / \mathrm{sec}$ to $30.5 \mathrm{Mc} / \mathrm{sec}$ and can be read to an accuracy of the order of $\pm 300 \mathrm{c} / \mathrm{sec}$. Thus, frequency was measured to an accuracy of the order of \pm 0.001 per cent. Due both to the upper frequency limit of the Collins receiver and to the decreased accuracy of impedance measurements at higher frequencies, most of the data were taken at or below the quarter-wave resonant frequency of the individual lines. All of these frequencies are below $31 \mathrm{Mc} / \mathrm{sec}$. This limitation of data is not a serious restriction since Fig. 14 shows that linear lines, which are operating at odd -multiple quarter-wave resonances above the first $(n=3,5, \ldots)$, have characteristics that very closely approach those of the uniform line. Hence, the interesting properties of linear lines are exhibited at frequencies up to the quarter $\backsim$ wave value.

The initial impedance measurements of the strip lines were made using the General Radio Type 916-A R-F Bridge which covers a frequency range of $400 \mathrm{Kc} / \mathrm{sec}$ to $60 \mathrm{Mc} / \mathrm{sec}$. The estimated accuracy of reactance measurements made with this bridge was $\pm(2 \%+1 \mathrm{ohm})$. 
Auxiliary instruments used with the 916-A bridge were a General Radio Type 605-B Standard Signal Generator and the Collins receiver mentioned above. Since the impedance level of the strip lines was low, the bridge method led to large measurement errors. Hence, another technique was employed to measure the reactance of the experimental lines.

A Measurements Corporation Model 59 Megacycle Meter operated as a grid dip oscillator was used to detect resonance of the circuit formed by the shorted line in parallel with a standard capacitance. A General Radio Type 722 - N Precision Capacitor was employed to supply the standard capacitance, while the Collins receiver was used to measure the frequency of the megacycle meter. Thus, the measurement of the input reactance of the line was obtained by the following procedure:

1. The megacycle meter was adjusted to a selected frequency and loosely coupled to the resonant circuit.

2. The Collins receiver, with the use of its beat frequency oscillator, was adjusted to zero beat with the megacycle meter.

3. Finally, the precision capacitor was adjusted to provide resonance at the selected frequency.

The proper setting of the capacitance was obtained by noting that as the circuit approached resonance the megacycle meter frequency was pulled slightly from its selected value thereby producing an audio signal in the receiver. At resonance the megacycle meter returned to its selected frequency and a null indication was obtained. From the frequency and the standard capacitance readings, the input reactance of the line was obtained. However, for precise results a correction was required. Without altering the leads to the capacitor, the input terminals of the line were shorted. The procedure outlined above was repeated for the circuit formed by the standard capacitance and the residual inductance due to the connecting leads. The frequency and capacitance readings determined the capacitive reactance which was set equal to the residual inductive reactance. This quantity was then 
subtracted from the original reading of input reactance as a correction. Since the precision capacitor has an accuracy of $\pm 1 \mathrm{mmf}+0.1$ per cent, and since the frequency error was negligible, the results were very good. The minimum value of the capacitance was $100 \mathrm{mmf}$. Hence, the accuracy of the reactance measurements is of the order of \pm 2 per cent or better. However, this method was not suitable for the openwire exponential line. As previously mentioned, the critical frequency of $12.1 \mathrm{Mc} / \mathrm{sec}$ required the input reactance measurements to be above that frequency. The minimum value of the precision capacitor resonated with this shorted exponential line at $7.6 \mathrm{Mc} / \mathrm{sec}$. Hence, measurements could not be obtained above $12.1 \mathrm{Mc} / \mathrm{sec}$. This difficulty illustrates the principal disadvantage of this method, namely, for the given shorted line the range of measurements was restricted to the frequency range determined by the minimum and maximum values of the available precision capacitance. With the standard capacitance removed, the quarter-wave resonant frequency was obtained by the above technique for all of the strip and open-wire lines. Experimental Results.--Fig. 26 shows the agreement between theoretical (solid lines) and experimental (circles) data for the four uniform strip lines. The measured data include the experimental errors and, therefore, indicate the correlation to expect for the similar linear lines. Fig. 27 shows the deviation (computed value minus measured value divided by computed value) for the two types of data. Taking into account the \pm 2 per cent measurement error, the agreement is quite good indicating that errors due to geometry are small.

Fig. 28 presents similar data for three of the linear strip lines. A uniform line is included for comparison. The spacing at the open end of all of these lines was maintained at $1 / 2$ inch to keep end effects at that end constant. The deviation, shown in Fig. 29, indicates that the linear line theory compares quite favorably with that of the uniform line. It is noted that, as the average spacing increases, the linear lines are more susceptible to fringing and end effect errors. Since the most accurate comparison is expected from the two lines with the smallest average spacings, Fig. 30,shows the results for these lines. 


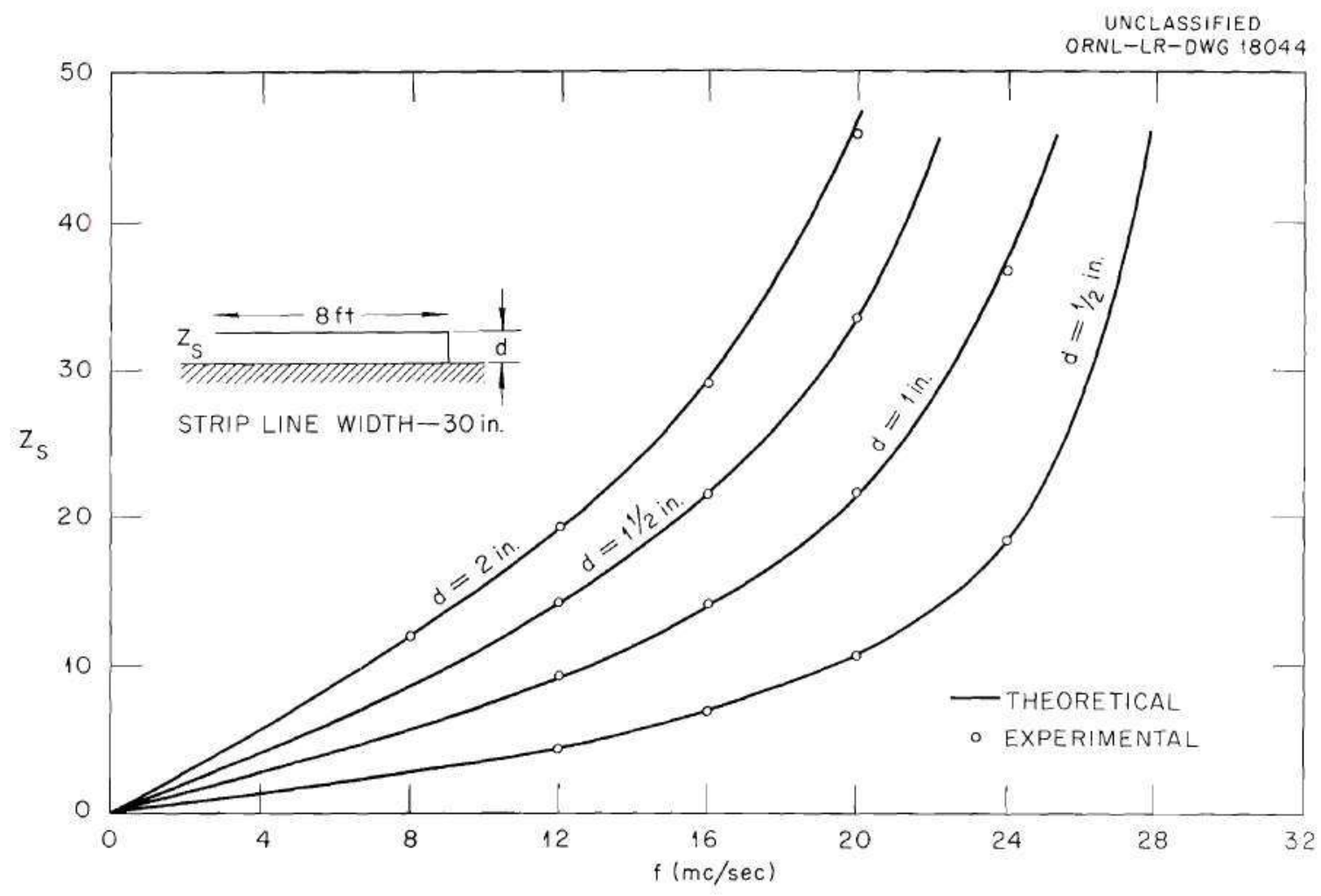

Fig. 26. Input Impedance vs. Frequency of the Uniform Strip Lines. 


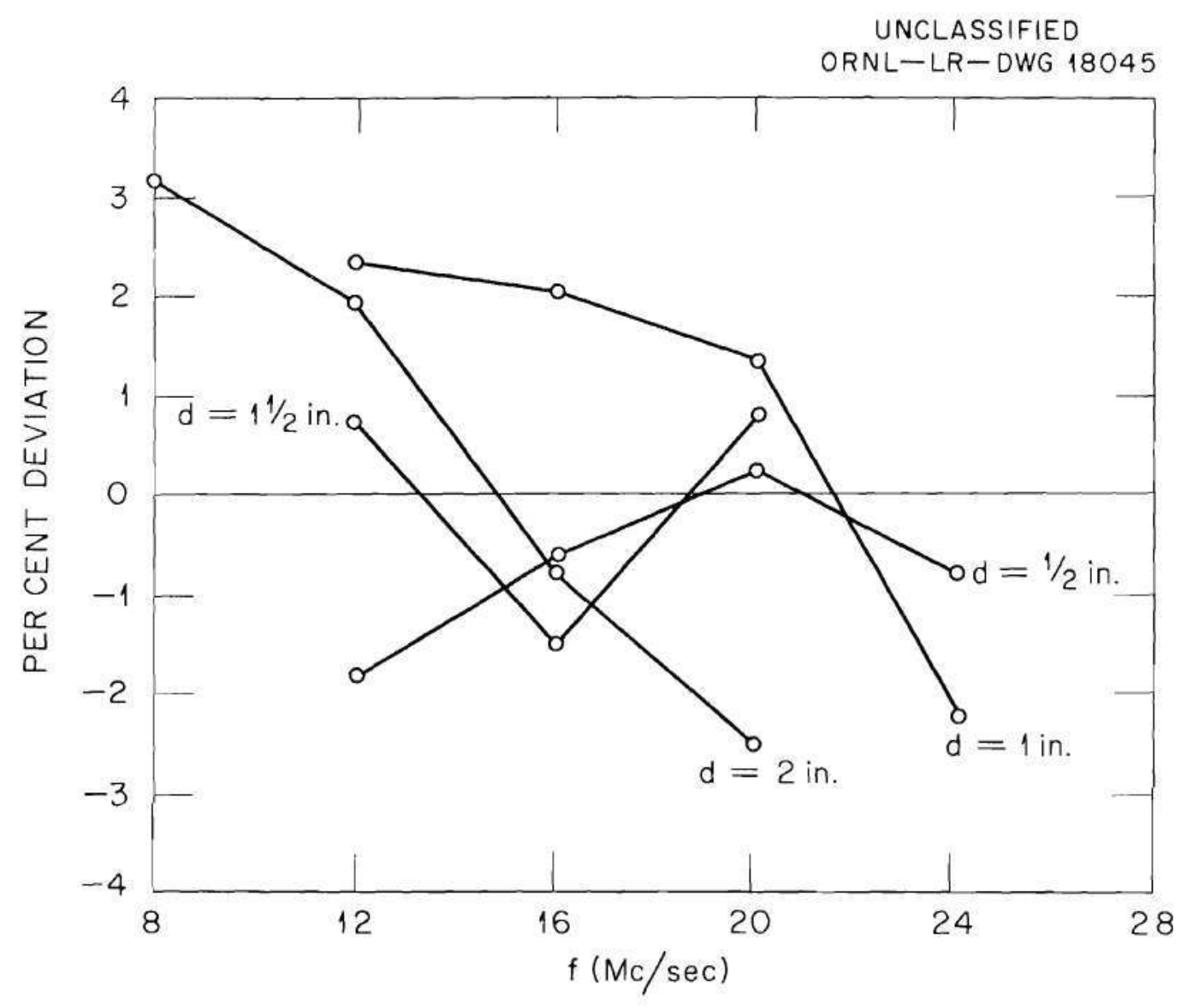

Fig. 27. Deviation vs. Frequency for the Four Uniform Strip Lines. 


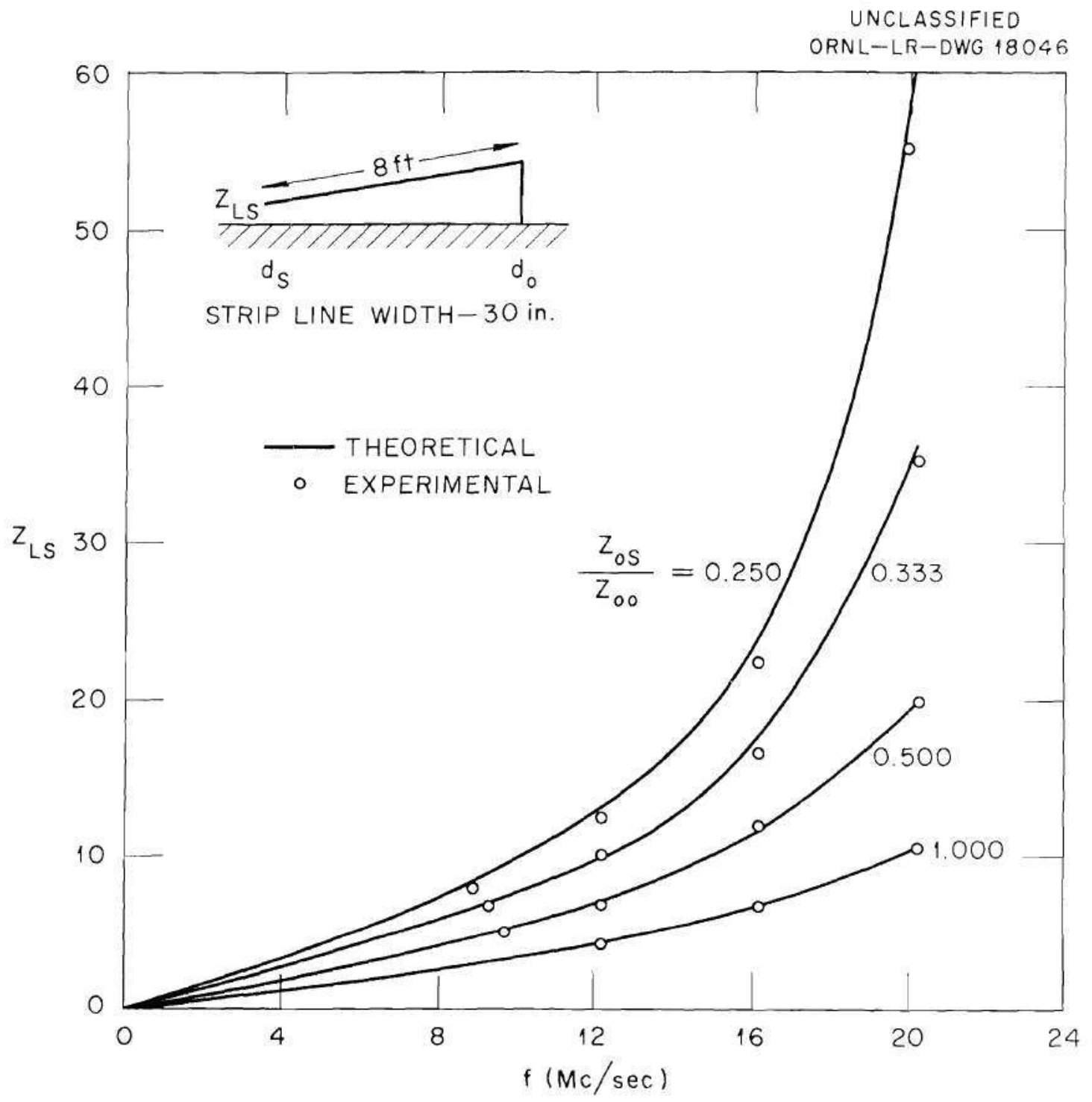

Fig. 28. Input Impedance vs. Frequency for the Four Linear Strip Lines. 


\section{$-88-$}

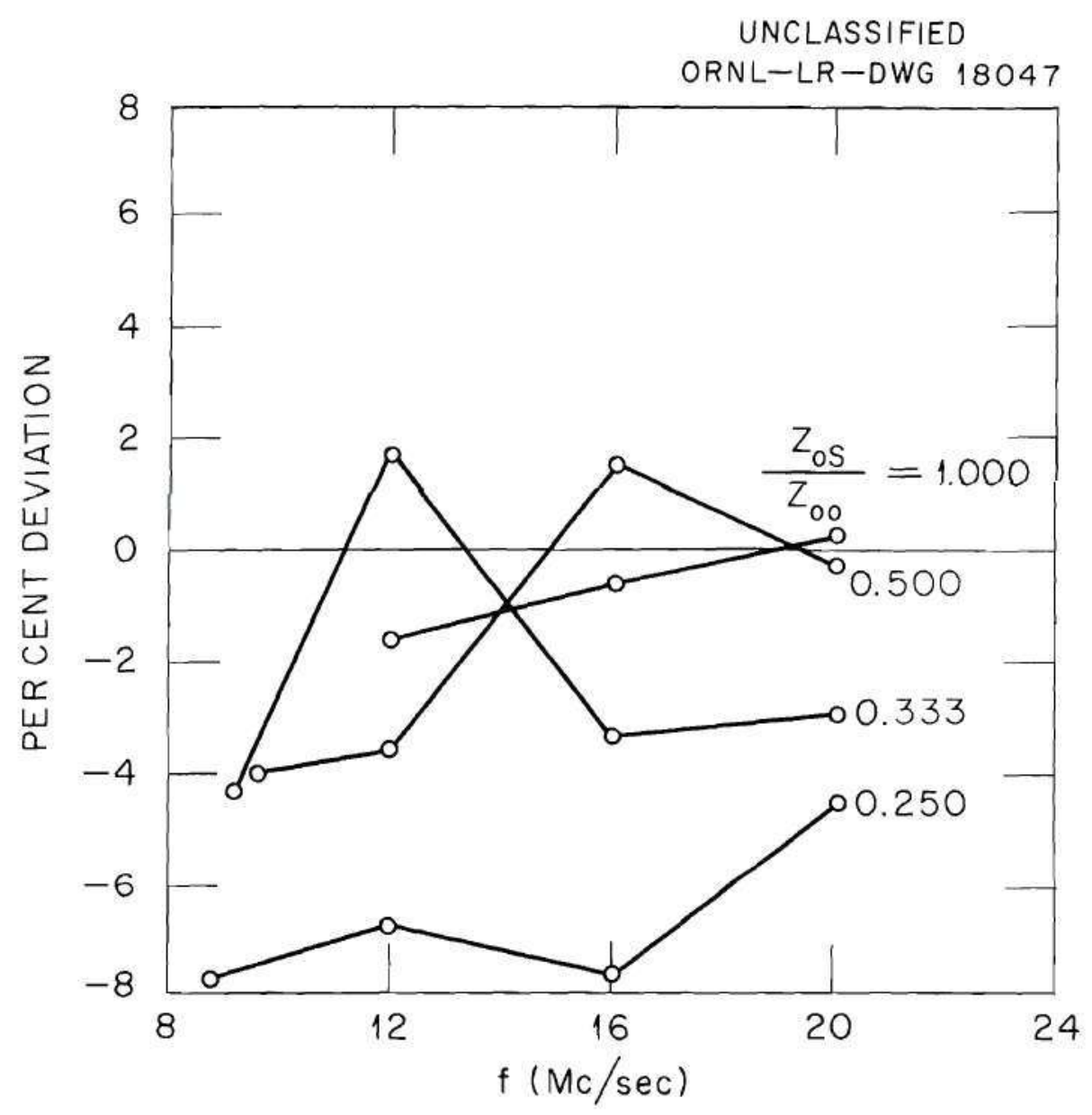

Fig. 29. Deviation vs Frequency for the Four Linear Strip Lines. 


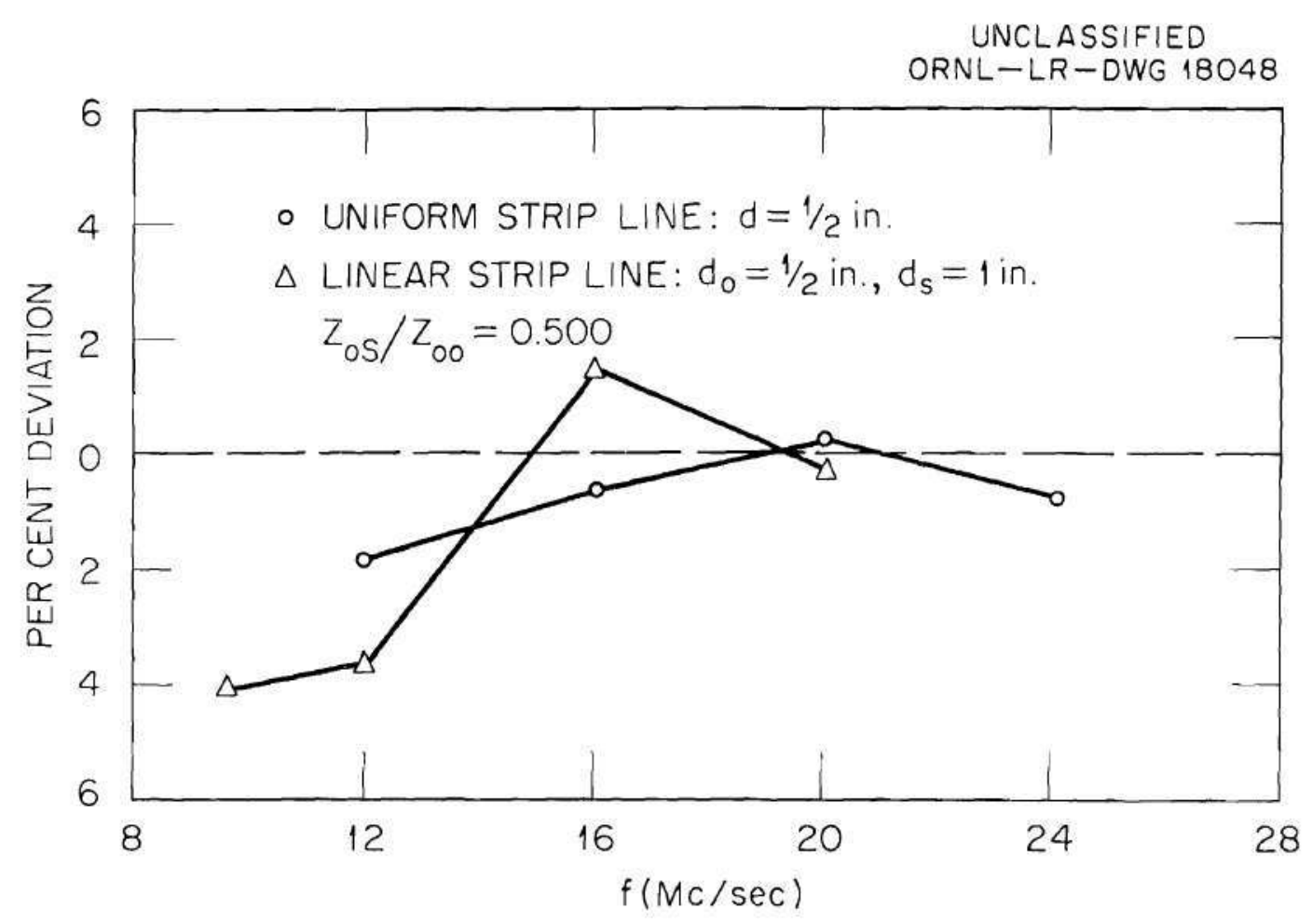

Fig. 30. Deviation vs. Frequency for a Linear and a Uniform Strip Line. 
Fig. 31 and 32 present similar results for the open-wire lines. Agair, the agreement is seen to be good.

Inaccuracies, which result from the application of the theory to physical.systems having relatively large end effect and geometrical inaccuracies, are common to both theories.

Table I shows the comparison between measured and computed values for the quarter-wave resonant frequency for all of the eight strip lines. As is expected, the deviation in frequency (defined in Table I) is smallest for the uniform strip line with the smallest spacing $\left(Z_{0}=6.28\right.$ ohms $)$. A similar statement is true for the linear strip lines. In both cases, the increase in deviation with increase in average spacing is attributed essentially to end effects which are not constant but depend upon the geometry of the line.

Principally for the purpose of determining what might be expected from experimental data, the velocity of propagation is computed from the measured frequency, $f_{o m}$, and the measured length, $(\ell+\mathrm{d} / 2)$, and is included as the ratio, $v^{\prime} / c$, for interest. (The actual velocity, of course, is assumed to be equal to the speed of light.) This computed ratio has the same trend as the frequency devi. ation since it is computed by using the measured frequencies. All of the strip lines are considered as having essentially the same velocity of propagation.

Finally, Table I presents similar results for the three open. wire lines. As is obtained from the uniform line, the computed velocity of propagation is seen to be $98.7 \%$ of the velocity of light. The experimental open-wire lines have two errors besides those possessed by the strip lines. One, as shown in Fig. 25, the spacing of the lines is maintained by lucite supports which affect the average velocity of propagation. Two, the spacing as a function of position is not exact since the line tends to consist of a series of straight segments (between supports) instead of the required continuous curve. These errors make the open-wire line less desirable for checking theory. However, the data presented tend to indicate the results that can be expected with open-wire lines. 


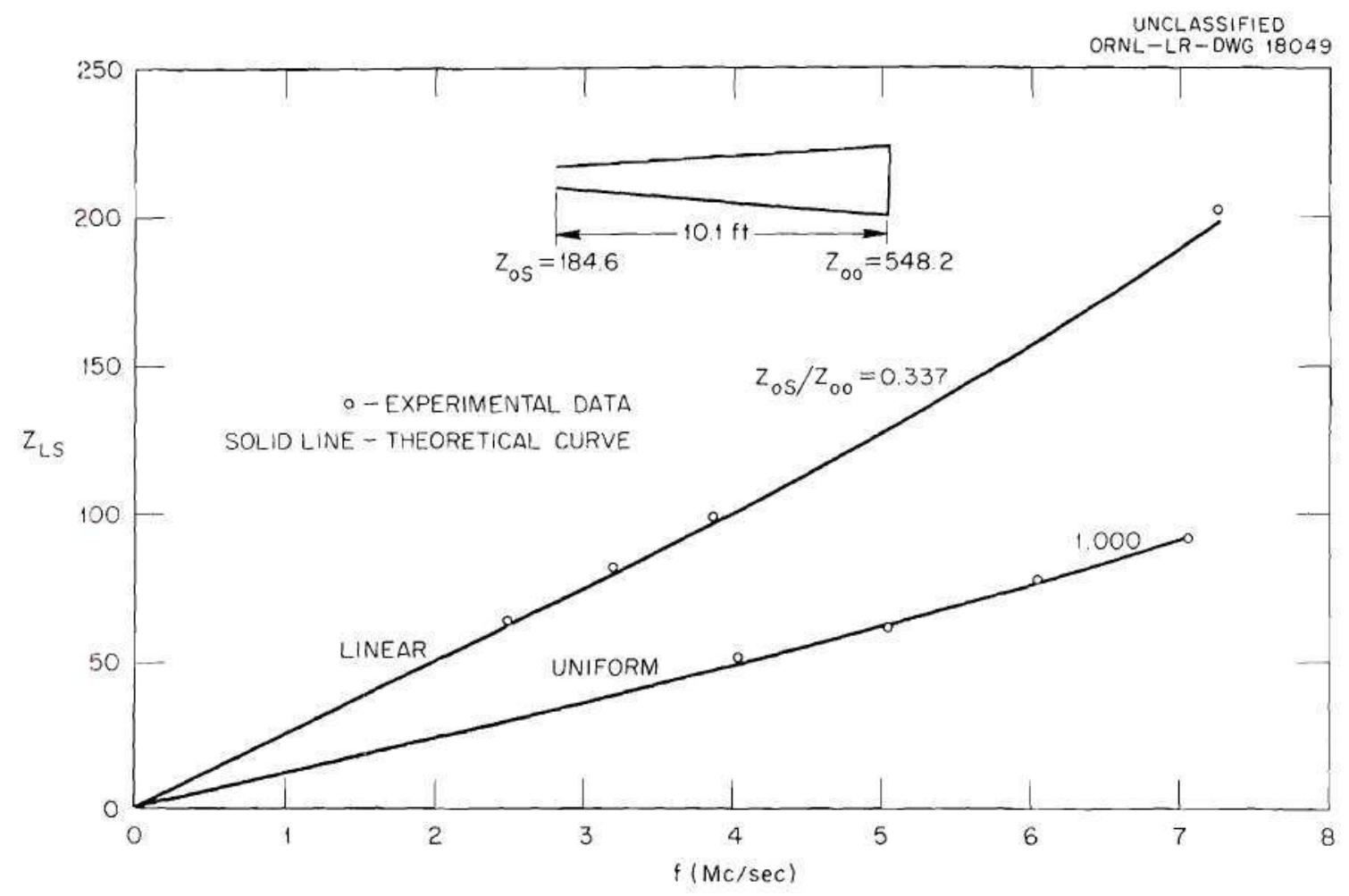

Fig. 31. Input Impedance vs. Frequency for a Linear and a Uniform Open Wire Line. 


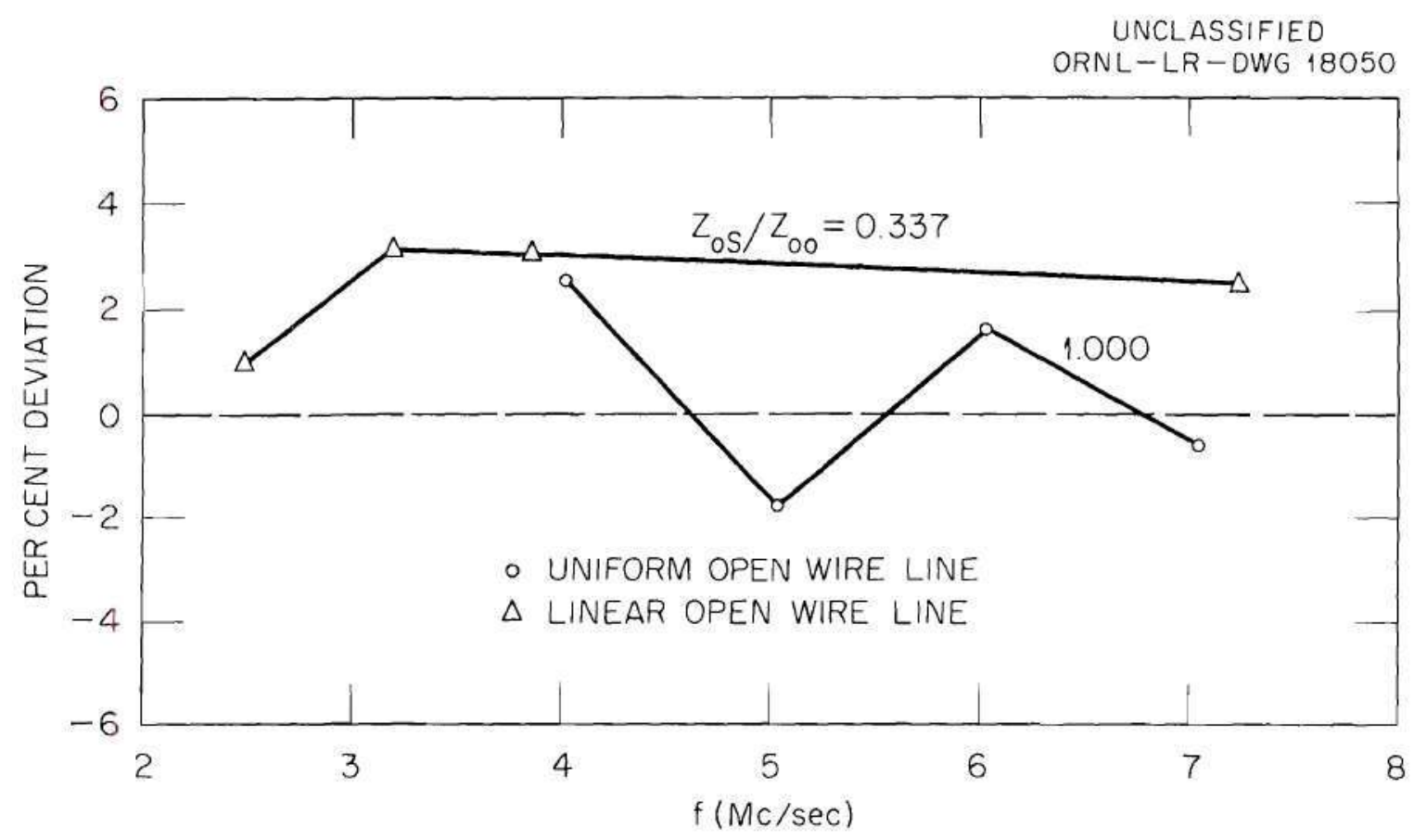

Fig. 32. Deviation vs. Frequency for a Linear and a Uniform Open Wire Line. 
TABLE I

COMPARISON OF MEASURED AND COMPUTED QUARTER-WAVE FREQUENCIES

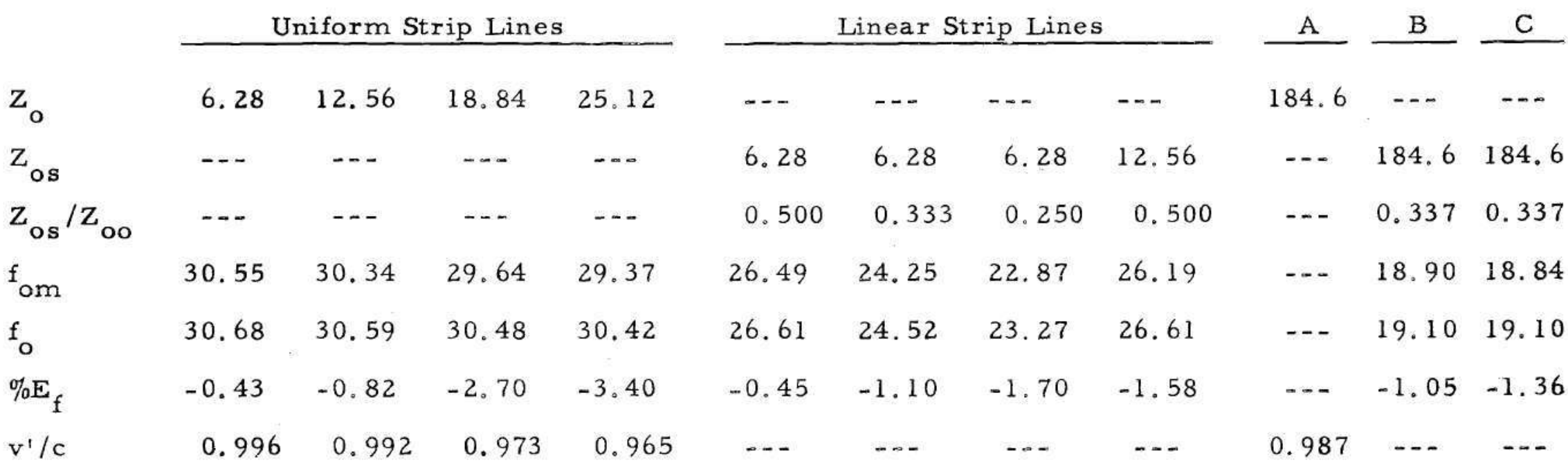

A - Uniform Open Wire Line

B - Linear Open Wire Line

C - Exponential Open Wire Line

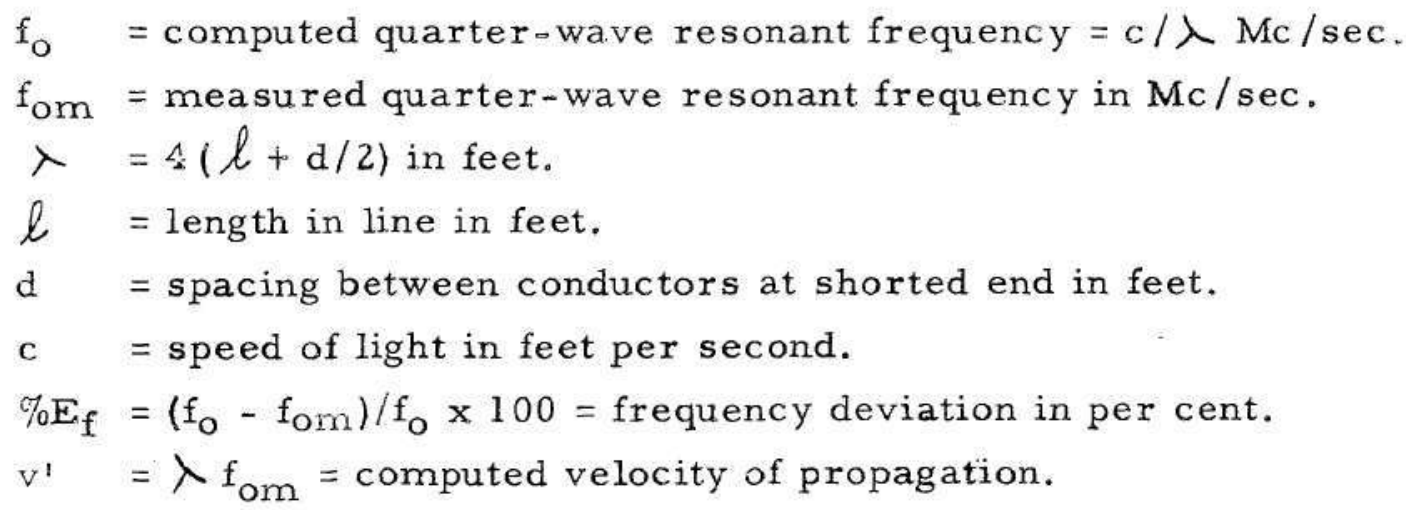


Discussion of Errors.--As previously mentioned, the three primary quantities that were measured were length, frequency, and impedance (reactance).

The lengths of the lines were measured to an estimated accuracy of $\pm 1 / 8$ inch with the use of an ordinary steel tape measure. Since the lines were all 8 feet or greater in length, the accuracy of these measurements was of the order of \pm 0.1 per cent. The spacing of the strip lines was estimated to be within 0.010 inch of the nominal spacing determined by the lucite strips. All of the lucite strips were integ ral multiples of $1 / 2$ inch within 0.001 inch as determined by a micrometer. Thus, since the smallest nominal. spacing was $1 / 2$ inch, the accuracy of these values was of the order of \pm 3 per cent or better. Spacing of the open-wire lines at the lucite spacers was accurate to $\pm 0.003 \mathrm{inch}$. Hence, the accuracy at these points was of the order of \pm 1 per cent since the minimum nominal spacing was 0.255 inch. However, since the open-wire line between spacers tended to be a series of straight segments instead of a continuous curve, the accuracy of the spacings at the supports was not particularly indicative of the accuracy of the entire line.

A.s noted in a previous section, frequency was measured with the Collins receiver to an accuracy of the order of \pm 0.001 per cent. This was the estimated accuracy of all. frequency measurements.

The accuracy of impedance measurements was estimated to be $\pm(2 \%+1 \mathrm{ohm})$ for the $916-\mathrm{A}$ bridge method and \pm 2 per cent for the $722-\mathrm{N}$ precision capacitor method. Due to the higher accuracy, the latter method was used to obtain all of the final data.

Discrepancies between measured and theoretical results were not directly indicated by the inaccuracies of the measurements of the primary quantities above. For example, the error between the meas ured and computed values of the input reactance for a given line was not only related to the measurement error but was also related to the errors due to the assumed values for the line parameters used in the computation, as well as to errors due to end effects. Since the last two types of errors mentioned were functions of the particular line, 
and since it was beyond the scope of this work to embark upon a lengthy study of errors associated with particular line geometries, an effort was not made to separate the different types of errors. Emphasis was placed upon reducing end effect and spacing inaccuracies to obtain reasonably accurate data. The correlation of results was based upon the comparison of data from linear lines with that of similar uniform lines which were subjected to comparable errors. Summary. - The procedure and apparatus used to verify the theoretical results obtained for the linear line in Chapter IV are described in this chapter. Experimental data are presented in the form of input impedance vs frequency and quarter-wave resonance vs taper for five linear lines. In addition, experimental data for comparable uniform lines are presented to correlate measurement errors.

Experimental data for one exponential line are included for completeness.

A discussion of the results presented in the two preceding chapters, including a discussion of the limitations of the theory, is given in the next chapter. 
CHAPTER VI

\section{DISCUSSION OF RESULTS}

Introduction. - - Results from Chapters III and IV are consolidated in this chapter by correlating the resonant properties of the exponential, linear, and uniform lines. The principal objectives are to present and discuss composite illustrations comparing analogous properties, as well as to consider the limitations of the theory.

In the interest of time, it was found necessary to limit the number of illustrations for discussion to those judged sufficient for indicating the typical. similarities and differences. For this reason, emphasis continues on the lossless shorted line. Since lossless open lines have relationships similar to those existing among the shorted lines, a discussion of the significant properties of the shorted lines will suffice for pointing out the general characteristics of the tapered lines.

Following the procedure used in Chapters III and IV in which the tapered lines were compared to the uniform line, composite results, comparing all three lines simultaneously, can be obtained by super position. Thus, the following characteristics are presented to illustrate the relative similarities and differences:

1. Relative voltage and current standing waves on a shorted quarter-wave line.

2. Normalized input impedance of a lossless shorted line as a function of length, and of frequency.

3. Relative resonant lengths for the three lines, operating at the same frequency, as a function of the taper.

4. Relative resonant frequencies for the three lines of equal length as a function of taper.

5. Relative power dissipation as a function of the taper for quarter-wave lines operating at the same frequency. 
6. Relative input resistance at resonance of a quasilossless line as a function of taper.

In addition, the principal equations describing the respective lines are listed in Appendix II for comparison. Comparison of the Three Lines.--Before proceeding with a discussion comparing the lines, it is necessary to define the basis for comparison. Unless specifically stated otherwise, the conditions for which the lines are compared are as follows:

1. A.ll lines are assumed to be shorted at one end and open at the other. Ordinarily the shorted end is designated as the receiving end for convenience.

2. Voltage and current distributions are normalized and related to each other by requiring that the voltages and the nominal characteristic impedances at the open ends are equal for all three lines.

See Fig. 33 for a schematic representation of the above conditions.

Relative voltage and current distribution curves are presented in Fig. 34 showing the typical shapes of the exponential and linear lines for tapers of $\mathrm{Z}_{\mathrm{oS}} / \mathrm{Z}_{\text {oo }}$ equal to 0.395 and 6.250 compared to cosinusoidal $\left(\mathrm{I}_{\mathrm{N}}\right)$ and sinusoidal $\left(\mathrm{V}_{\mathrm{N}}\right)$ distributions for the uniform line. Fig. 35 is included to show more details of the current distribution of the linear line, while Fig. 36 shows the approximate boundary* curves for current and voltage. Curves for the exponential line, analogous to those in Fig. 36, are shown in Fig. 37.

The relative magnitude and shape of the current curves for the positive exponential line $\left(\mathrm{Z}_{O S} / \mathrm{Z}_{0 o}\right.$ is less than unity) are very nearly equal to those for the linear line as is indicated in Fig. 34. However, the limiting current curve (not shown) in this region is for $\mathrm{Z}_{\mathrm{OS}} / \mathrm{Z}_{\mathrm{oo}}$ equal to 0.135 which can be shown to correspond to the condition that the operating resonant frequency is equal to the critical frequency for the line. Thus, none of the curves to be shown exist for values of $\mathrm{Z}_{\mathrm{oS}} / \mathrm{Z}_{\text {oo }}$ less than 0.135 , since this would require an operating frequency

*That is, $\mathrm{Z}_{\mathrm{oS}} / \mathrm{Z}_{\text {oo }}$ equal to very large and very small values. 
UNCLASSIFIED

ORNL-LR-DWG 18051
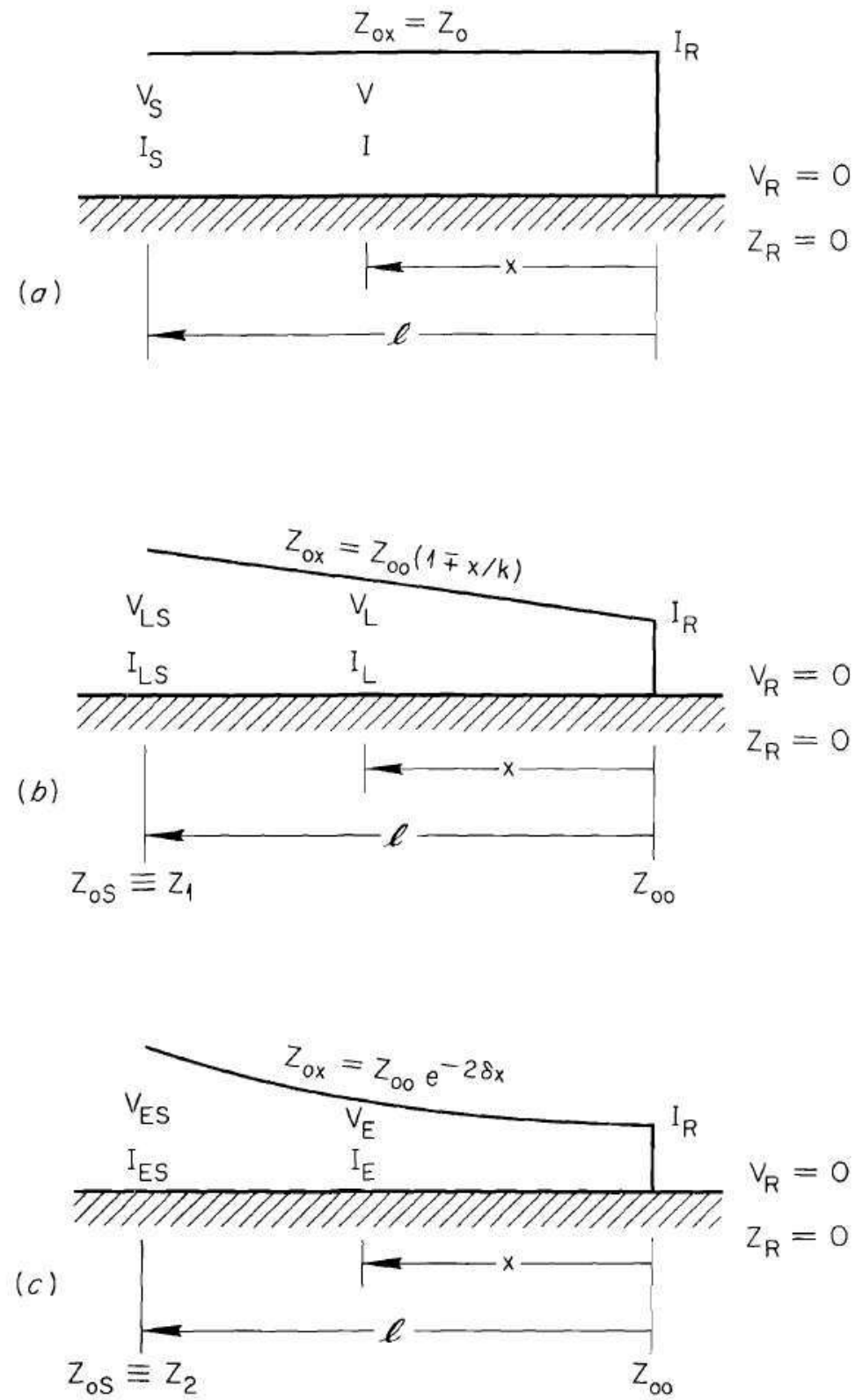

Fig. 33. Basis for Comparing the (a) Uniform, $(b)$ Linear, and $(c)$ Exponential Lines:

$$
\mathrm{V}_{\mathrm{S}}=\mathrm{V}_{\mathrm{LS}}=\mathrm{V}_{\mathrm{ES}} ; \mathrm{Z}_{0}=\mathrm{Z}_{1}=\mathrm{Z}_{2} \text {. }
$$


UNCLASSIFIED

ORNL-LR-DWG 18052

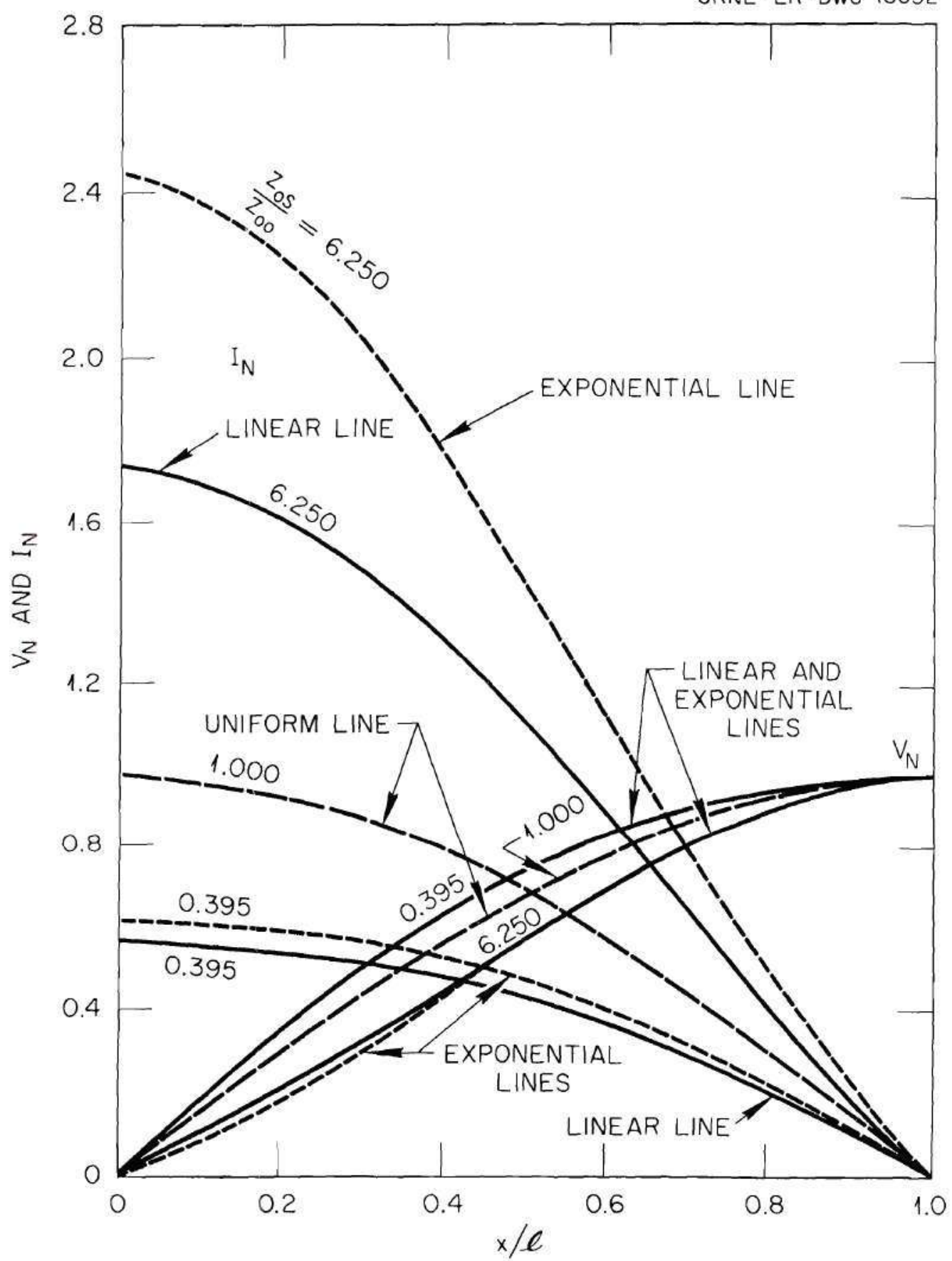

Fig. 34. Composite Relative Voltage and Current Distribution Curves vs Normalized Length for the Linear and Exponential Lines. 


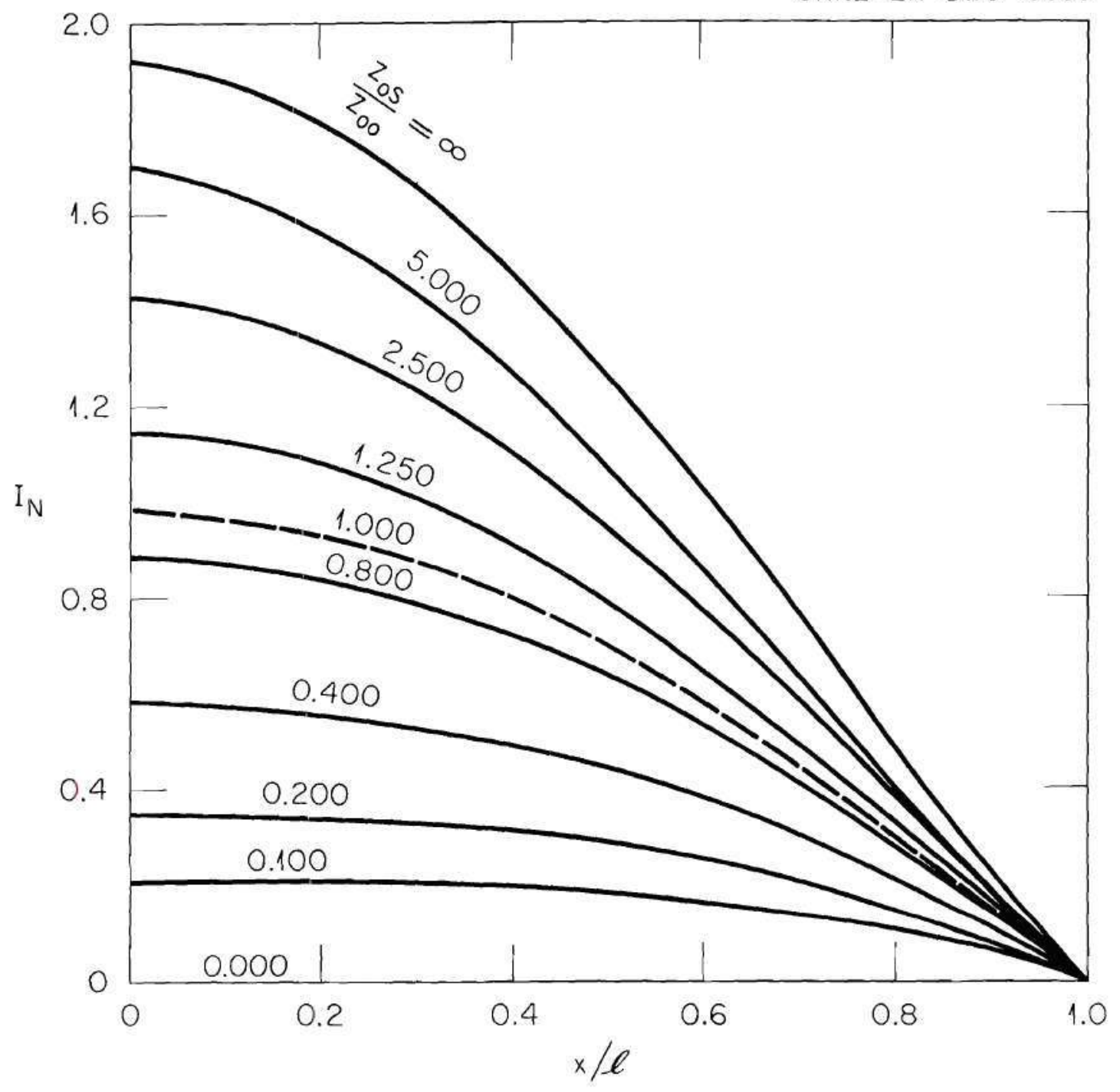

Fig. 35. Relative Current Distribution vs Normalized Length for the Linear Line. 


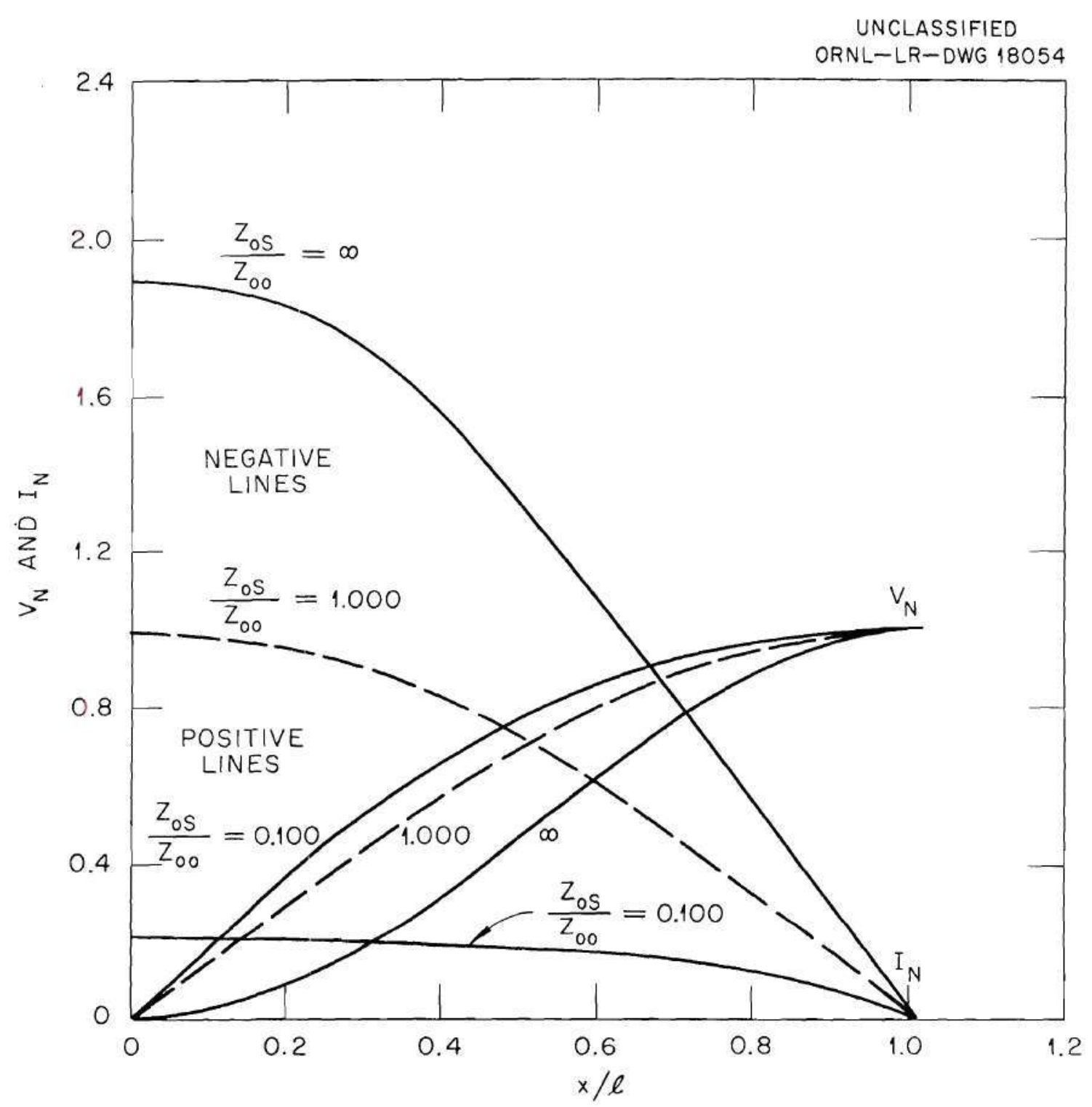

Fig. 36. Relative Voltage and Current Distribution vs. Normalized Length for the Linear Line. 


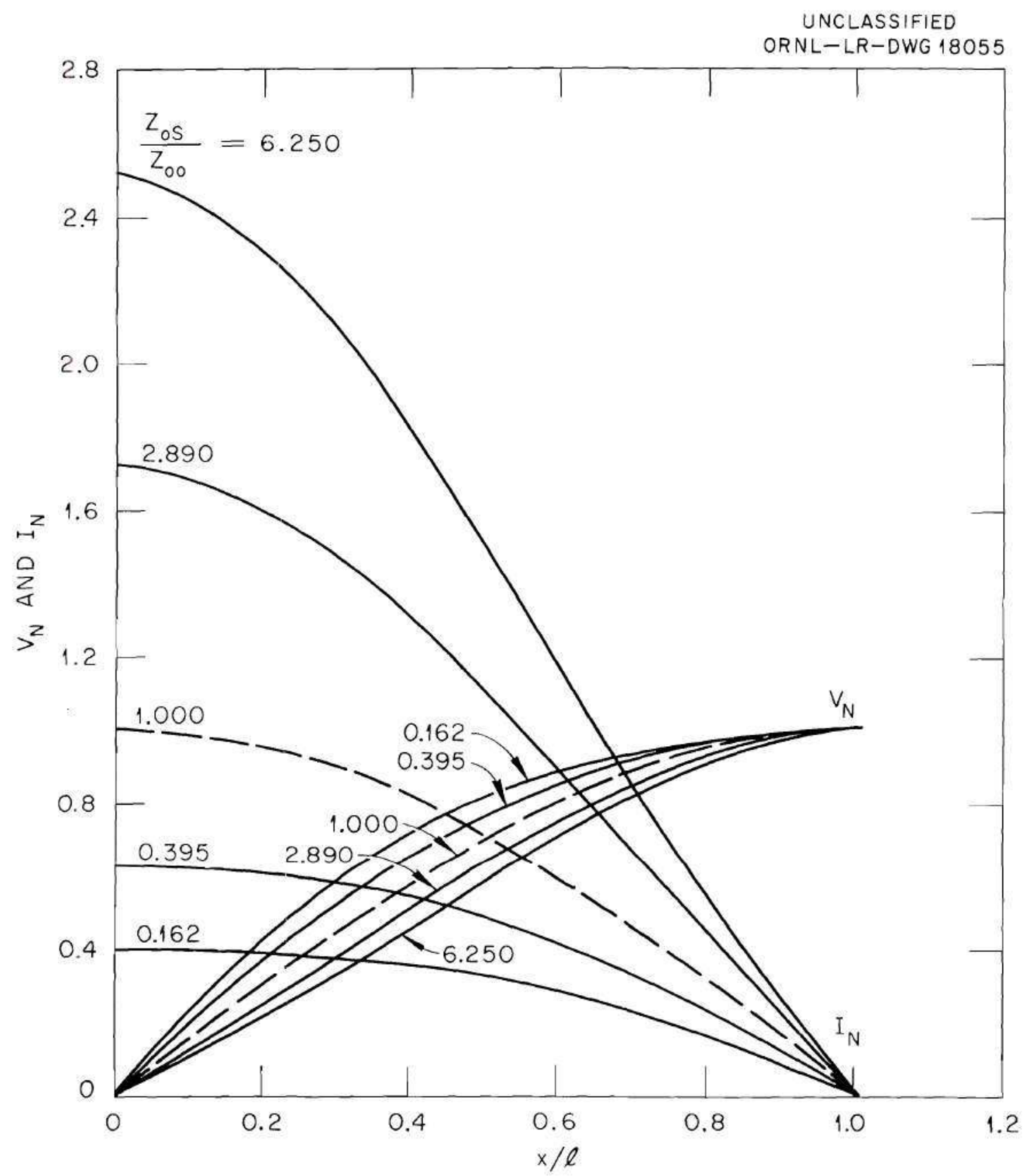

Fig. 37. Relative Voltage and Current Distribution vs. Normalized Length for the Exponential Line. 
below the critical frequency. A careful study of Fig. 35 and 37 indicates that the curve marked $\mathrm{Z}_{\mathrm{oS}} / \mathrm{Z}_{\text {oo }}$ equals 0.200 (Fig. 35) roughly approximates the limiting curve corresponding to $\mathrm{Z}_{\mathrm{oS}} / \mathrm{Z}_{\text {oo }}$ equals 0.135 for the exponential line. As a comparison, the analogous limiting curve for the linear line is obtained for the value $\mathrm{Z}_{o S} / \mathrm{Z}_{o \circ}$ equal to zero ( $F i g$. 35) which corresponds to $I_{N}$ equal to zero. For $\mathrm{Z}_{o S} / \mathrm{Z}_{\text {oo }}$ equal to unity, all three lines have the same curve, of course.

In the region of the negative line, the shapes of the linear and exponential lines are essentially the same except the magnitudes diverge (Fig. 34). In the limit, $x / l$ equal to zero, the magnitude of the current for the linear line approaches $1.93\left(\mathrm{Z}_{\mathrm{oS}} / \mathrm{Z}_{\mathrm{oo}}\right.$ approaches infinity) while that of the exponential line increases without limit (Fig. 36 and 37).

The corresponding voltage curves, as is indicated in Fig. 34, are essentially the same for both lines except for very large values of $\mathrm{Z}_{\mathrm{OS}} / \mathrm{Z}_{\mathrm{OO}}{ }^{\circ}$

The normalized input impedance of a lossless shorted line, which is the ratio of voltage to current in Fig. 34, is considered next. Fig. 38 shows the impedance curves for the same values of $\mathrm{Z}_{\mathrm{OS}} / \mathrm{Z}_{\mathrm{oo}}$ used in Fig. 34. The curves are similar in general shape to a circular tangent $\left(\mathrm{Z}_{\mathrm{OS}} / \mathrm{Z}_{\mathrm{OO}}\right.$ equals unity) but deviate from this curve as shown. As may be expected from studying the voltage and current (Fig. 34), the impedance for the positive line has the same shape and magnitude for both types of tapered lines. However for the negative line, the similarity in shapes continues, but the magnitudes begin to diverge as $\mathrm{Z}_{\mathrm{oS}} / \mathrm{Z}_{\text {oo }}$ becomes large. The data in Fig. 38 is replotted in a different manner in Fig. 39 by changing the scale of the abscissa. This scale in Fig. 38 is a relative length as referred to the total length of line. (Specifically, it is the distance, $\underline{x}$, along the line divided by the total length of the line.) This total length is understood to be the value for quarter-wave resonance for the given value of $\mathrm{Z}_{\mathrm{OS}} / \mathrm{Z}_{\mathrm{oo}}$ and the given frequency - all lines being resonant at the same frequency. In Fig. 39 the scale is changed to an absolute scale as referred to the leng th of the uniform line. The asymptotes of the individual curves indicate the resonant lengths of the different lines relative to that of the uniform line. 


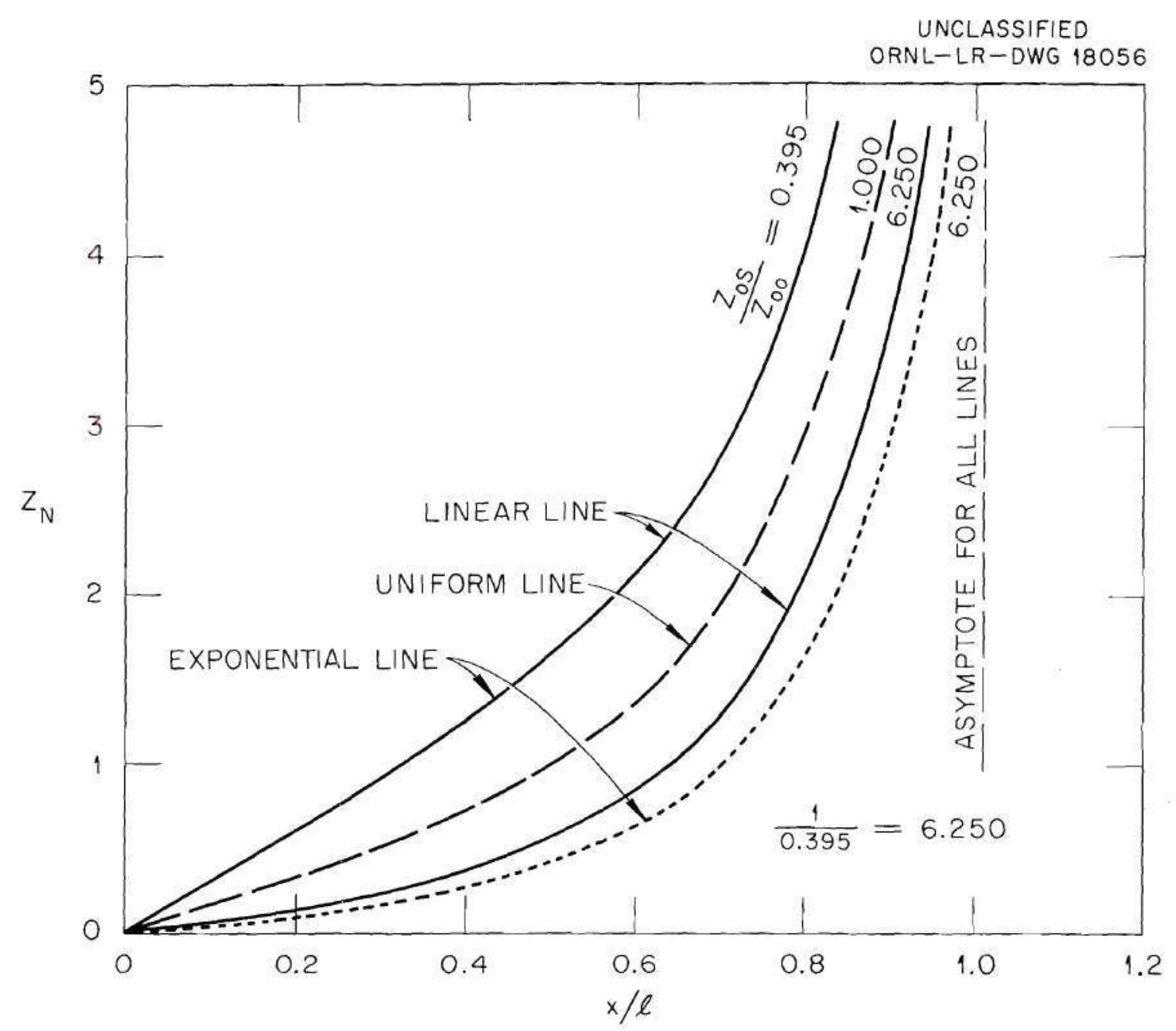

Fig. 38. Composite Curves of Normalized Input Impedance vs. Normalized Length for Lossless Linear and Exponential Lines. 


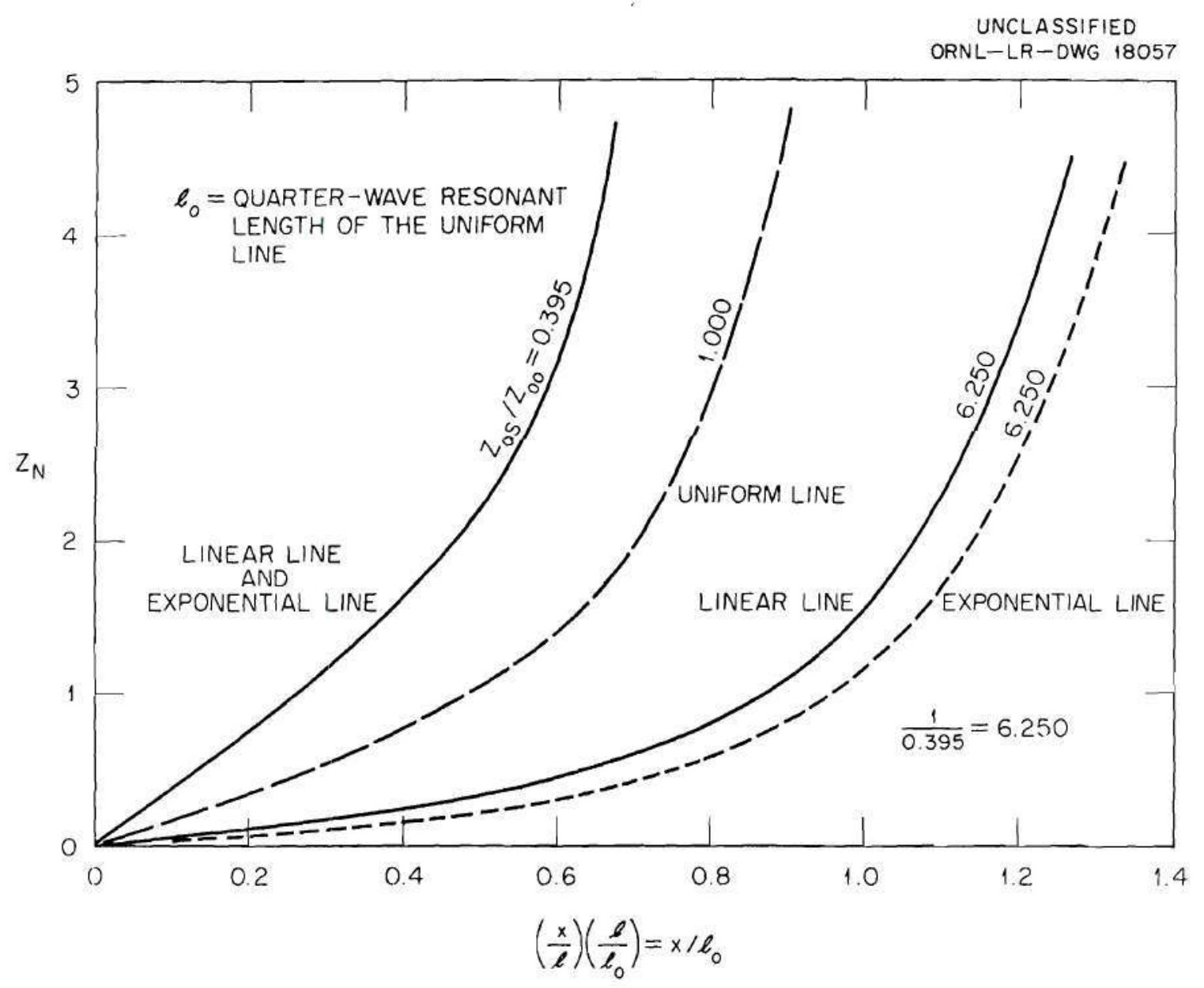

Fig. 39. Composite Curves of Normalized input Impedance vs. Length for Lossless Linear and Exponential Lines. 
To clarify the above statements, consider a uniform line operating in quarter-wave resonance at $24.6 \mathrm{Mc} / \mathrm{sec}$. The free space wave length is 40 feet. Thus, the quarter-wave length of the uniform line is 10 feet. Then, $x / l_{0}$ equal to 1.000 corresponds to 10 feet absolute. From this information it is easy to determine that $x / l_{0}$ equal to 0.822 corresponds to 8.22 feet. Therefore, a tapered line (either an exponential or a linear line when the curves are coincident) with $\mathrm{Z}_{o S} / \mathrm{Z}_{o o}$ equal to 0.395 will be 8.22 feet in length when in resonance at $24.6 \mathrm{Mc} / \mathrm{sec}$. As a further illustration, consider the value of the normalized input impedance of a section of this line defined by $x / l$, equal to 0.400 which corresponds to 4.00 feet. At this abscissa, $Z_{N}$ is equal 1.62. Then if the nominal characteristic impedance at the open end, $Z_{o S}$ is equal to $100 \mathrm{ohms}$ at $24.6 \mathrm{Mc} / \mathrm{sec}$. A corresponding leng th of uniform line with the same characteristic impedance ( $100 \mathrm{ohms}$ ) will have an absolute input impedance equal to $74 \mathrm{ohms}$ at $24.6 \mathrm{Mc} / \mathrm{sec}$.

It is to be noted that the scale factor, $l / l_{0}$, by which the abscissa of Fig. 38 is multiplied to obtain that of Fig. 39, is taken from the curves in Fig. 40, It is especially to be noted in Fig. 40 that, for values of $\mathrm{Z}_{\mathrm{Oo}} / \mathrm{Z}_{\mathrm{oS}}$ above 0.200 for the negative line and for values of $\mathrm{Z}_{\mathrm{oS}} / \mathrm{Z}_{\text {oo }}$ above 0.300 for the positive line, the two curves are essentially coincident. That is, for all practical purposes over the range of $\mathrm{Z}_{\mathrm{Oo}} / \mathrm{Z}_{\mathrm{OS}}$ and $\mathrm{Z}_{\mathrm{OS}} / \mathrm{Z}_{\mathrm{O}}$ described, the exponential and linear lines behave identically with respect to resonant lengths or resonant frequencies. Hence, a shorted linear and exponential line, which have equal lengths and equal $\mathrm{Z}_{\circ \mathrm{S}} / \mathrm{Z}_{00}$ ratios, will resonate at the same frequency. The resonance is a function of the impedance ratio and is independent of whether the nominal characteristic impedance varies linearly or exponentially from $Z_{o o}$ to $Z_{o S^{\circ}}$ This suggests, but does not prove, that all lines whose impedance may be made to vary monotonically from $Z_{\text {oo }}$ to $\mathrm{Z}_{\mathrm{OS}}$ and to lie between the linear and exponential curves may exhibit the same resonant properties. The range specified, including both negative and positive lines, is substantial. That is, expressed as follows:

$$
0.3 \leq z_{\mathrm{oS}} / \mathrm{z}_{\mathrm{oO}} \leq 5.0 .
$$




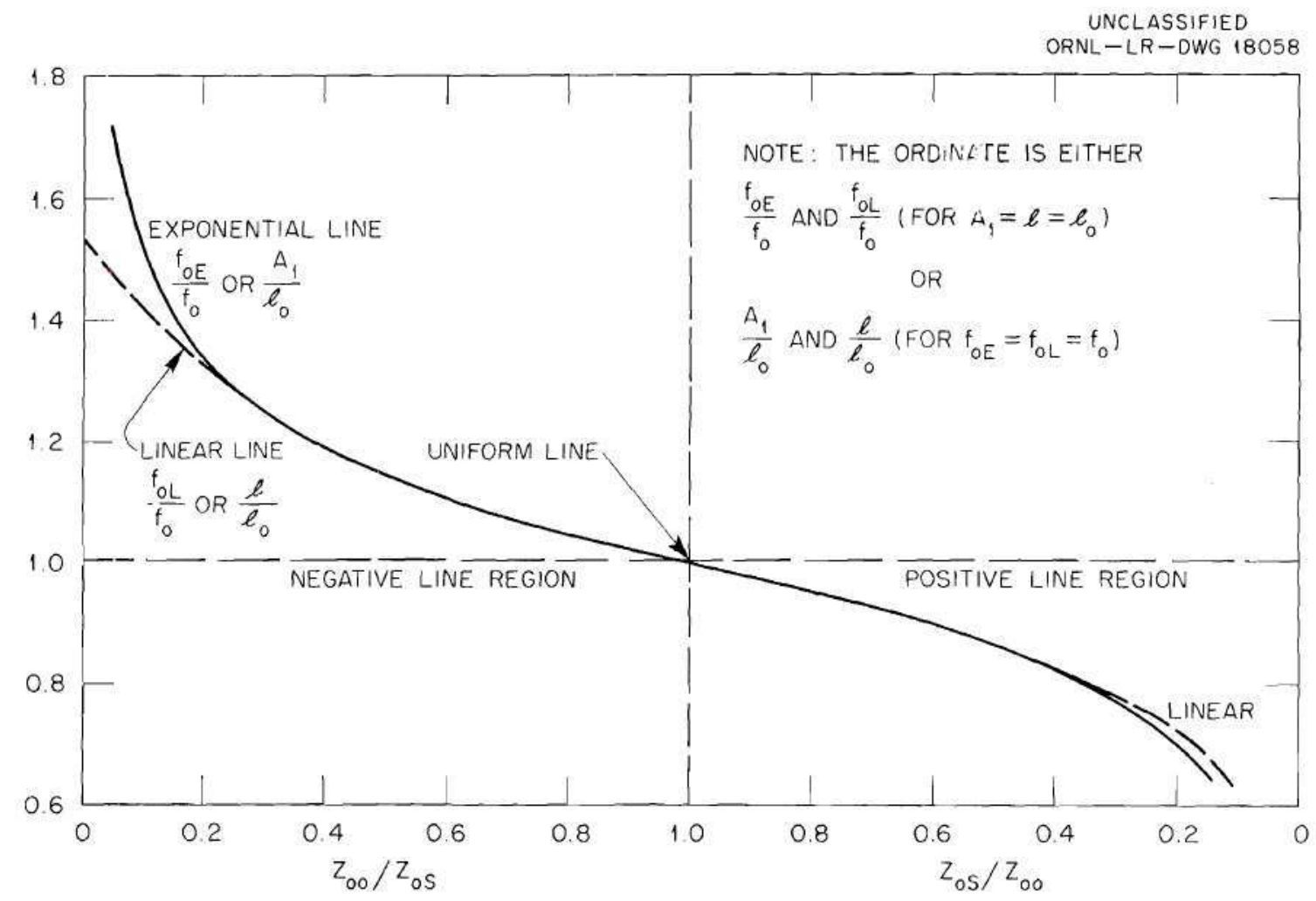

Fig. 40. Quarter-Wave Resonance vs. Taper of the Lossless Linear and Exponential Lines. 
A line with $\mathrm{Z}_{\mathrm{oS}} / \mathrm{Z}_{\text {oo }}$ equal to 5.0 represents an appreciable change in impedance from one end to the other. Hence, the range over which this interesting property is valid is not trivial. As for the regions

$$
0.0 \leq z_{o o} / z_{o S} \leq 0.2
$$

for the negative line, and

$$
0.0 \leq \mathrm{Z}_{\mathrm{oS}} / \mathrm{Z}_{\mathrm{Oo}} \leq 0.3
$$

for the positive line, the curves diverge. The divergence, however, is very small for the positive line and not great for the negative line until $\mathrm{z}_{\text {oo }} / \mathrm{Z}_{\mathrm{oS}}$ is below 0.1. Thus, the two types of tapered lines have remarkably similar quarter-wave resonance properties over most of the range of $\mathrm{Z}_{\mathrm{OS}} / \mathrm{Z}_{\mathrm{oo}}$.

Fig. 41 presents power loss curves in the same manner as the resonant curves of the preceding figure. The ordinate represents a ratio of the power loss in a quarter-wave tapered line to that of a uniform line where both lines have the same voltage and nominal charac. teristic impedance at the open end, have the same constant resistance per unit length, and are operating at the same frequency. The coodinates (1.0,1.0) represent the uniform line. It is seen immediately that the power losses in a positive line rapidly diminish from that of the uniform line as $Z_{o S} / Z_{\text {oo }}$ departs from unity. For example, at $\mathrm{Z}_{\mathrm{oS}} / \mathrm{Z}_{\text {oo }}$ equal to 0.6 the power ratio is 0.55 . Hence, the power loss in the tapered line is 55 per cent of that in the uniform line.

The curves in Fig. 41 also represent the ratio of resonant impedances of the uniform line to the tapered line as seen at the open end of quasi-lossless lines. This is true since the power ratio already discussed may be shown to be equal to the reciprocal of the resonant impedance ratio. That is,

$$
P_{o L}=\left(V_{o S}\right)^{2} / 2 R_{p}
$$




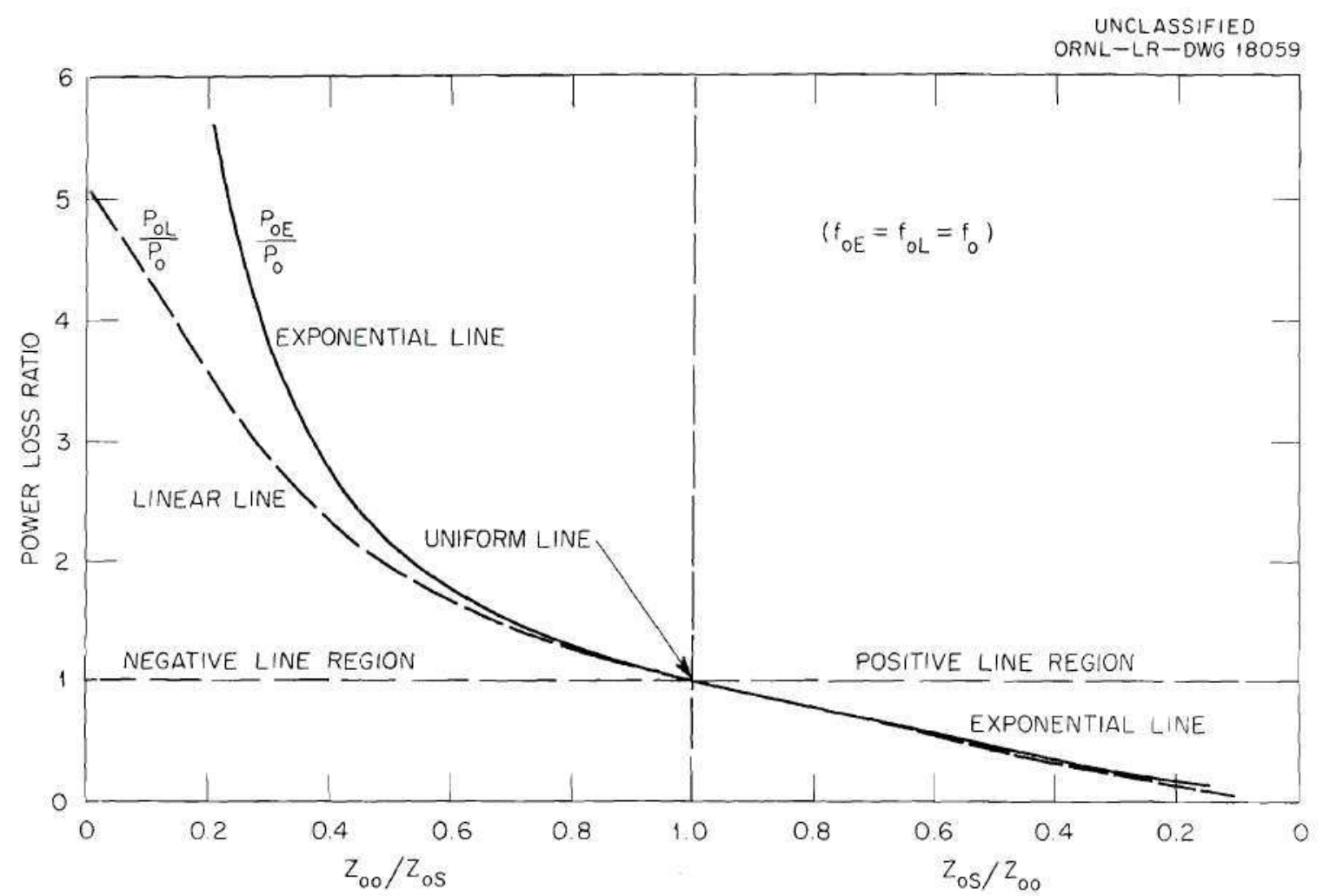

Fig. 41. Power Loss Ratio vs. Taper of the Quarter-Wave Linear and Exponential Lines. 
where $V_{o S}$ is the peak voltage at the open end and $R_{p}$ is the equivalent parallel resistance at resonance as seen at the open end of the line. Hence,

$$
R_{p} / R_{p o}=\frac{\left(V_{o S}\right)^{2} / 2 P_{o L}}{\left(V_{o}\right)^{2} / 2 P_{o}}=P_{o} / P_{o L}
$$

which is the reciprocal of the power ratio. As an illustration, consider the example mentioned above in which the power ratio was 0.55 . Thus,

$$
\mathrm{R}_{\mathrm{po}} / \mathrm{R}_{\mathrm{p}}=1 / 0.55=1.82
$$

and the resonant resistance of the tapered line is 1.82 times that of the corresponding uniform line.

The next group of curves presented in Fig. 42 and 43 shows the normalized input impedance of the shorted lossless lines as a function of frequency with both normalized and absolute scales similar to the presentation in Fig. 38 and 39. A study of Fig. 38, 39, 42, and 43 shows that the normalized input impedances are very similar whether a function of frequency or of length. The impedance curves for the exponential line are not shown for frequencies below the critical frequency corresponding to the value of $\mathrm{z}_{\mathrm{oS}} / \mathrm{Z}_{\text {oo }}$ involved. This feature is the principal difference between the two sets of impedance curves, one vs frequency and the other vs length.

Generally speaking, the linear line exhibits voltage, current, impedance, and power characteristic quite similar to those of the exponential line. Both types of tapered lines have properties significantly different in detail from those of the uniform line.

As a final illustration. Appendix II lists the principal equations of the three lines for comparison. A study of this list serves to indicate the reduction in complexity obtained by emphasizing the lossless case as a basis for comparing the analogous properties of the three lines. 


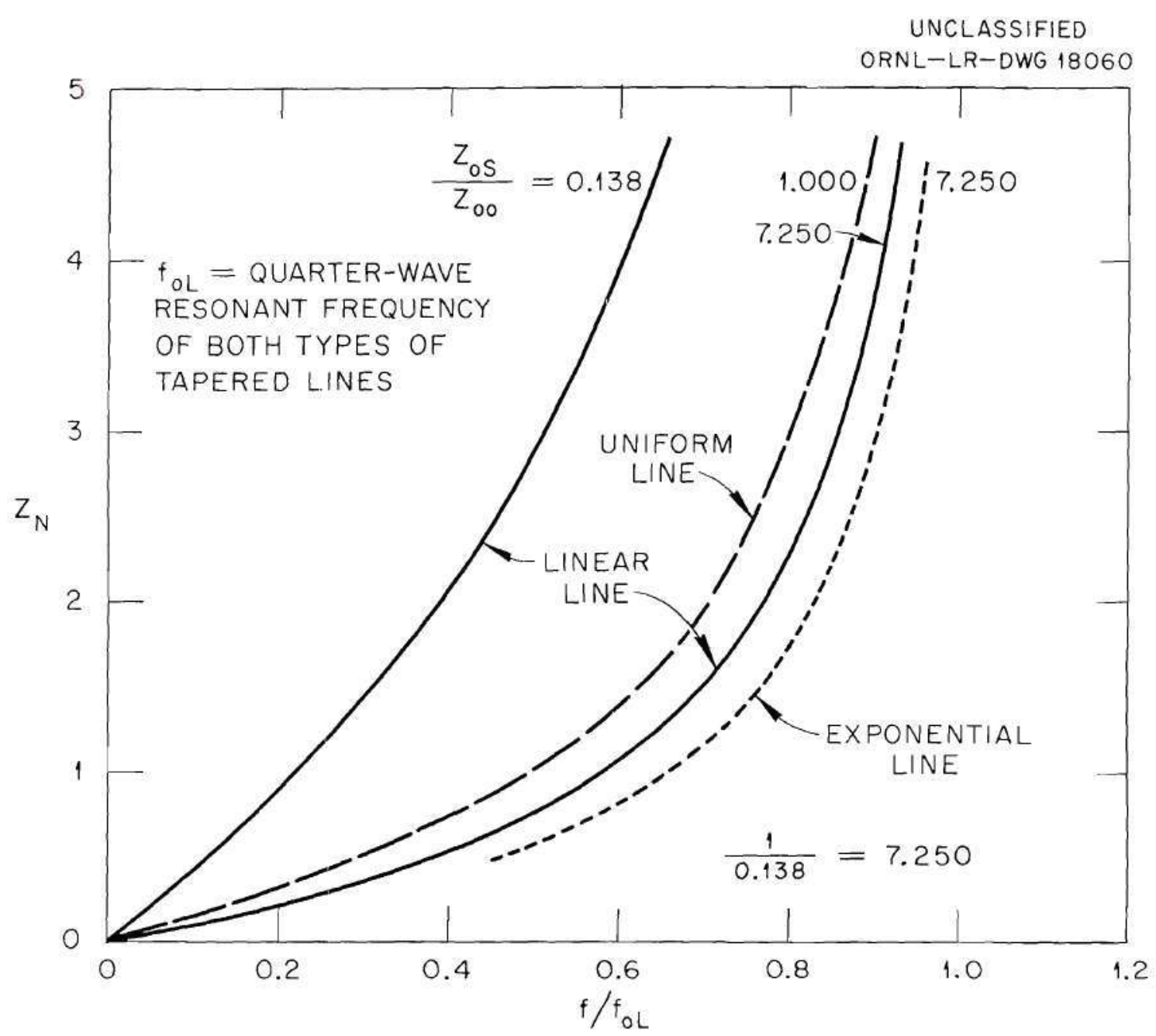

Fig. 42. Normalized Input Impedance vs. Normalized Frequellcy of Lossless Linear and Exponential Lines. 


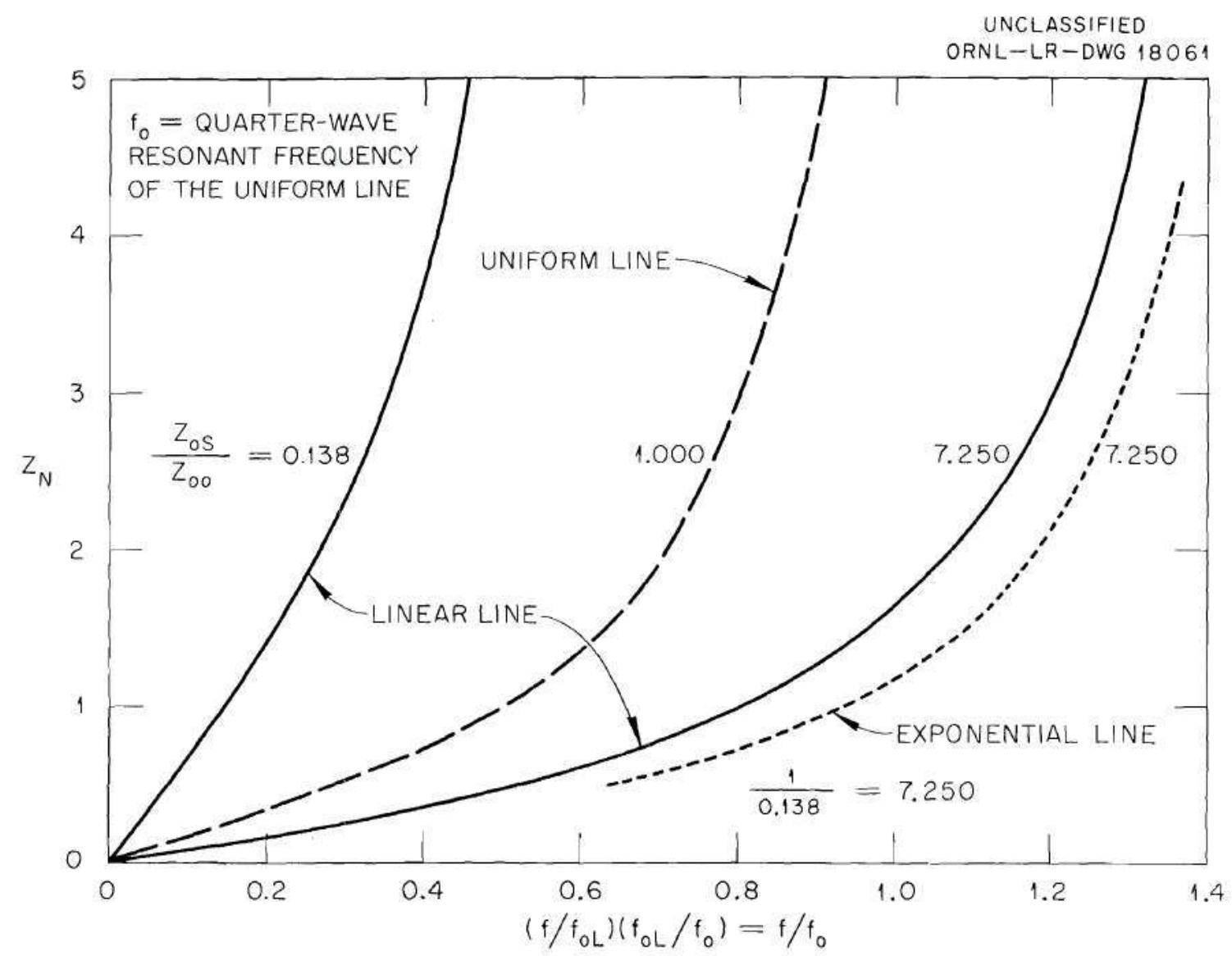

Fig. 43. Normalized Input Impedance vs. Frequency of Lossless Linear and Exponential Lines. 
Limitations of the Theory.--The preceding mathematical development of the tapered lines presents general equations for voltage and current which are subject to the following assumptions:

1. The analysis of the lines by ordinary transmission line theory is valid.

2. The lines have the assumed variations in the line constants, $R, L, G$, and $C$.

Simplified equations are obtained from the general equations by the additional as sumption:

3. The lines are lossless, that is,

$$
\mathrm{R}=\mathrm{G}=0
$$

Approximate expressions for the power loss of low-loss lines, defined by

$$
\begin{aligned}
& \mathrm{R}<\omega \pi, \\
& \mathrm{G}=0,
\end{aligned}
$$

are developed with the further assumptions:

4. The current distribution for the low-loss line is the same as that for the lossless line.

5. The power is given by integrating the square of the $\mathrm{r}-\mathrm{m}$-s current times the resistance per unit length of the line.

6. The resistance per unit length of the line is constant.

The first assumption involves the relationships among the voltage drop per unit length, the current, and the series impedance per unit length of line, and similarly, the change in current per unit length, the voltage between conductors, and the shunt admittance per unit leng th of line. This implies a transverse electromagnetic mode (TEM) of propagation to insure that neither an electric nor a magnetic field component exists in the direction of propagation. As a result, this mode permits the definition of a series impedance and a shunt admittance per unit length of line.

The second assumption merely requires that the assumed variations in the line constants are physically realizable in general. 
These first two assumptions are basic and will be discussed further below. The remaining assumptions ( 3 through 6) are forced conditions used to obtained simplified approximate results. In general, they are the same assumptions well known in the literature on uniform lines* and will not be discussed further.

To determine the order of magnitude of error introduced by the first two assumptions, consider a cylindrical wave guided by two semiinfinite conducting planes enclosing an angle $\phi$, as shown in Fig. 44. such that there is no variation in $\mathrm{E}_{\phi}$ or $\mathrm{H}_{\mathrm{x}}$ in the $\underline{x}$ direction. Then the electric field lines are along arcs of concentric circles and the equipotential planes are along radii and pass through the $\mathrm{x}$-axis. The analysis of this type of wave propagation by Maxwell's equations in Appendix III shows that the solutions for the electric and magnetic field intensities may be modified and expressed as total voltage and current expressions. Furthermore, the resulting forms of these equations are identical, respectively, with those obtained for the general lossless linear line in Chapter IV. Hence, Fig. 44 illustrates the geometry of a particular linear line; however if the geometry in Fig. 44 were used in connection with the theory in Chapter IV, two differences would be noted. One, the field lines in Chapter IV would be chords instead of arcs of circles as shown. Two, the distance $\underline{r}$ from the origin to the particular field line (arc) would not be the same as the distance $\underline{u}$ from the origin to the equivalent field line (chord). Thus, since the analysis by field theory techniques is essentially exact, the theory in Chapter IV is in error. The magnitude of such errors may be estimated by the method which follows.

When the potential differences between corresponding points $P_{I}$ and $\mathrm{P}_{2}$ are assumed to be the same for both methods of analysis, the error in nominal characteristic impedance may be obtained. From the field theory analysis

$$
\mathrm{z}_{\mathrm{or}}=377(\phi \mathrm{r} / \mathrm{w})
$$

*See, for example, W. C. Johnson, Transmission Lines and Networks, New York: McGraw-Hill Book Company, Inc., 1950. p. 145. 


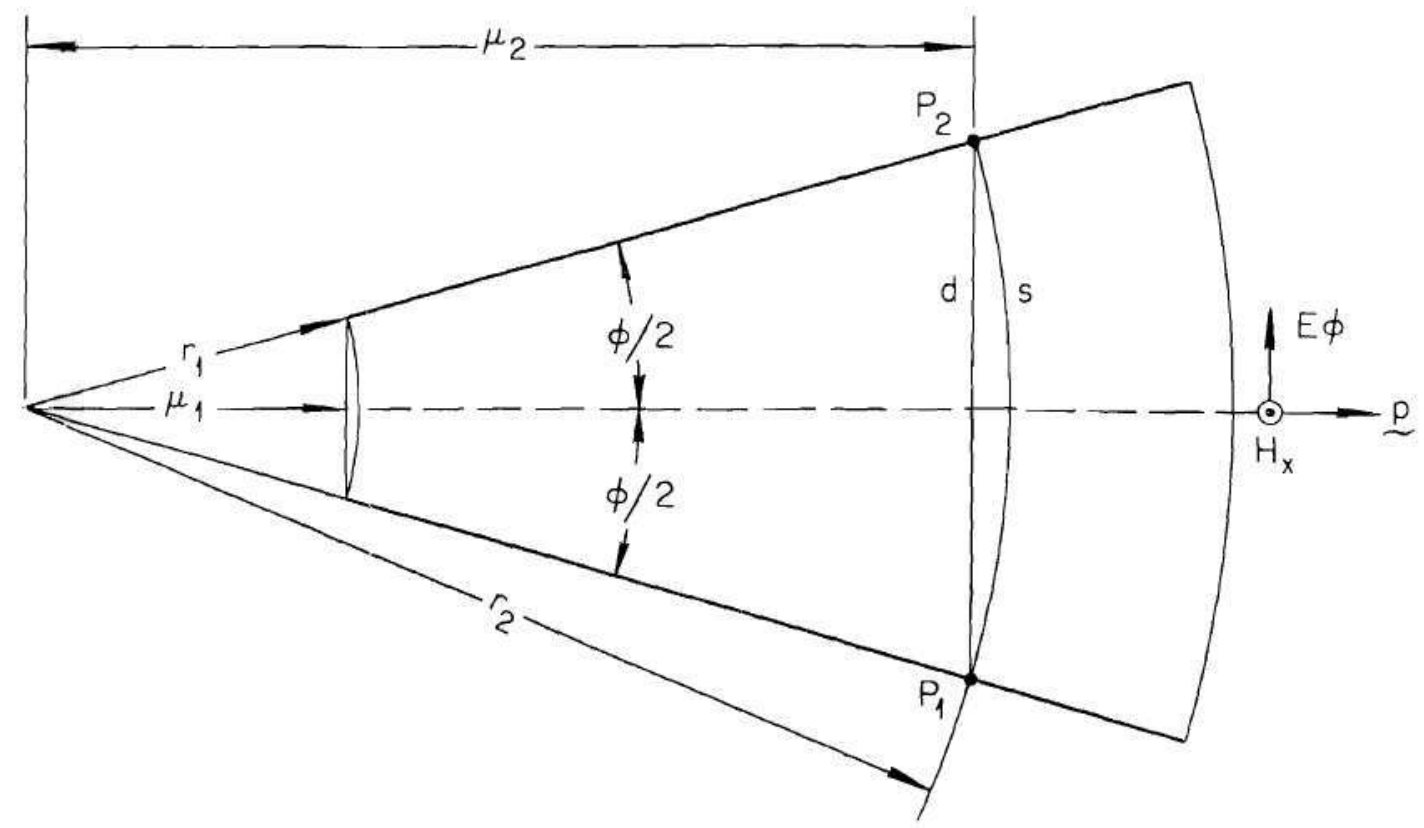

Fig. 44. Propagation of a Cylindrical Wave between Semi-Infinite Conducting Planes. 
where $\underline{s}$ is the distance between conductors along an arc at radius $\underline{r}$ and $\underline{w}$ is the width of the conductors. Similarly, when the parallel strip line equation is used (with the notation of Fig. 44)

$$
\mathrm{Z}_{\mathrm{ou}}=377(\mathrm{~d} / \mathrm{w})
$$

where $\underline{d}$ isthe distance between conductors along the chord at position $\underline{u}$. From (274) and (275), the error in nominal characteristic impedance is

$$
z_{\text {or }}-z_{\text {ou }}=(377 / w)(s-d)
$$

From trigonometry,

$$
\mathrm{s}=\phi \mathrm{r}
$$

and

$$
\sin (\phi / 2)=d / 2 r
$$

so

$$
s-d=r \cdot[\phi-2 \sin (\phi / 2)]
$$

and

$$
E_{z}=\left(z_{\text {or }}-z_{\text {ou }}\right) / z_{\text {or }}=\left[1-\frac{2 \sin (\phi / 2)}{\phi}\right] \text {. }
$$

Hence, for small $\phi$

$$
E_{Z} \rightarrow 0
$$

Next, consider the difference in the length of a section of line using the two methods. From the notation in Fig. 44, the length of the line, 
$\ell$, is

$$
\mathrm{u}_{2}-\mathrm{u}_{1}=\ell
$$

by the method of Chapter IV while the comparable quantity from the field theory method is

$$
r_{2}-r_{1}=\bar{l} .
$$

Now,

$$
u_{1} / r_{1}=u_{2} / r_{2}=\cos (\phi / 2)
$$

hence

$$
u_{2}-u_{1}=\left(r_{2}-r_{1}\right) \cos (\phi / 2)
$$

and

$$
\ell / \bar{l}=\cos (\phi / 2)
$$

so that the error is given by

$$
\mathrm{E}_{\ell} \equiv\left(\frac{\bar{l}-\ell}{\bar{l}}=[1-\cos (\phi / 2)]\right.
$$

Although equations (280) and (284) are developed for the linearly tapered strip line, they may be used to estimate the error for other types of tapered lines, both strip and coaxial. (The equations will be less accurate for open wire lines.)

Consider an incremental length of line as shown in Fig. 45. In the plane containing the axes of the two conductors, the electric field lines will approximate arcs of circles. Hence, (280) and (284) may be applied over the increment. The total error, $E_{\ell}$, over the length of 


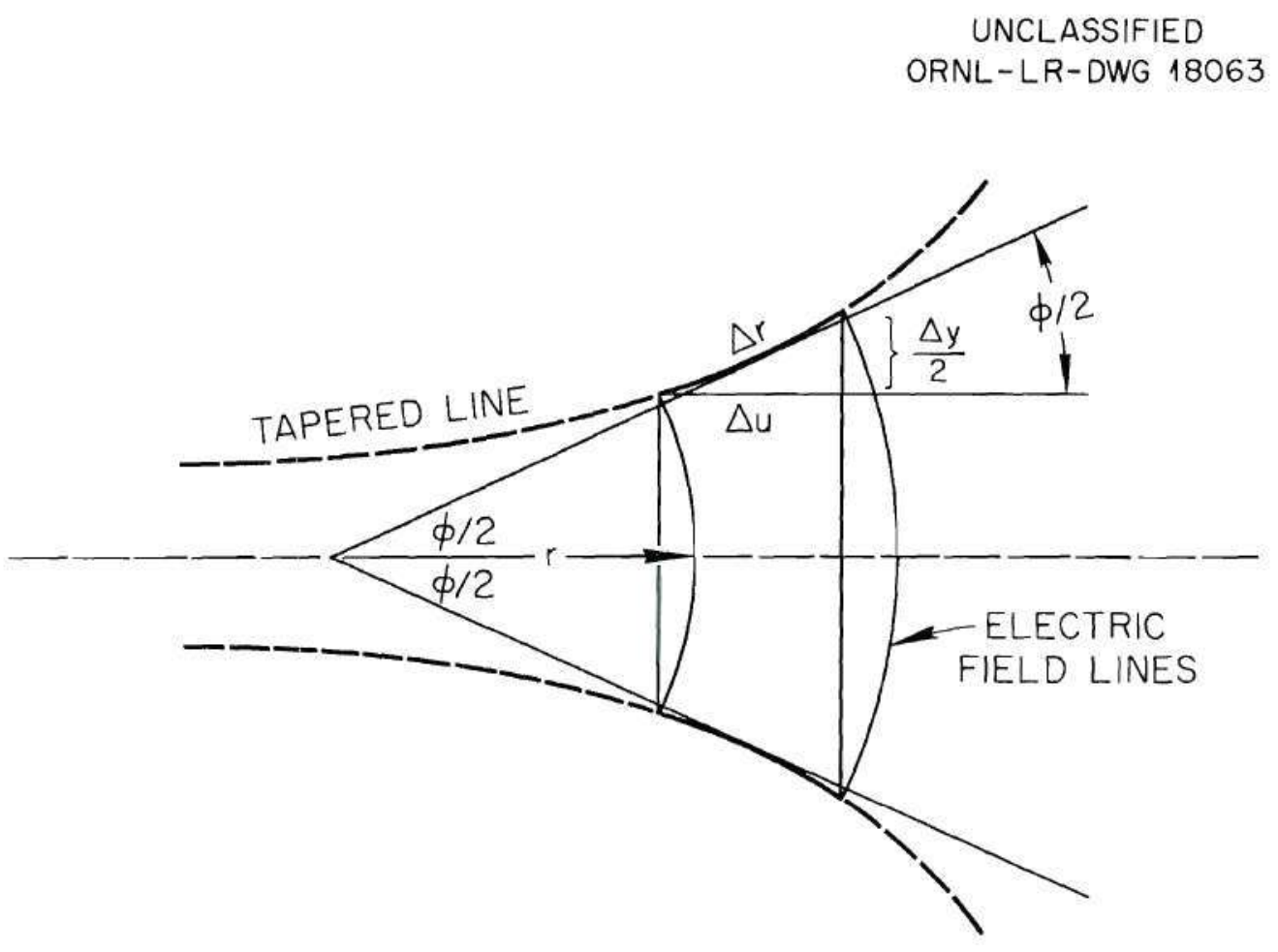

Fig. 45. An Incremental Length of Tapered Line. 
line

$$
l=u_{2}-u_{1}
$$

may be obtained as follows:

$$
\begin{aligned}
& \Delta \mathrm{u} / \Delta \mathrm{r}=\cos (\phi / 2)=\Delta \mathrm{u} / \sqrt{(\Delta \mathrm{u})^{2}+(1 / 4)(\Delta \mathrm{y})^{2}} \\
& \Delta \mathrm{E}_{\ell}=(\Delta \mathrm{r}-\Delta \mathrm{u}) / \Delta \mathrm{r} \\
& \mathrm{E}_{\ell} \equiv \int_{\mu_{1}}^{\mu_{2}} \mathrm{dE}_{l}=\int_{\mu_{1}}^{\mu_{2}}(\mathrm{dr}-\mathrm{du})(\mathrm{du} / \mathrm{dr})
\end{aligned}
$$

or

$$
E_{\ell}=\int_{\mu_{1}}^{\mu_{2}}\left[1-1 / \sqrt{1+(1 / 4)(d y / d u)^{2}}\right] d u
$$

Since

$$
\mathrm{dy} / \mathrm{du}=\mathrm{f}(\mathrm{u}) \quad
$$

(289) may be integrated in principle to obtain $\mathrm{E}_{\ell}$. The development above may be summarized by saying that the results presented in this investigation apply to tapered lines whose rate of taper with respect to wave length is small. This statement is essentially equivalent to saying that $\phi$ in (280) and (284) is small.

It is necessary to note the manner in which both $E_{Z}$ and $E_{\ell}$ affect the accuracy of the results. Errors in the nominal characteristic impedance produce errors in the results predicted by the theory approximately directly proportional to $\mathrm{E}_{\mathrm{Z}}$. That is, if $\mathrm{E}_{\mathrm{Z}}$ is equal to 1 per cent, the input impedance will be in error by approximately 1 per cent also. The effects of errors in length, however, are not easily determined since they depend upon the rate of change of input impedance (voltage or current) with length and therefore depend upon the manner in which the mathematical functions describing the behavior of the line are affected. 
Near quarter-wave resonance, for example, a given error in length corresponds to a much larger error in input impedance than for the case in which the line length is remote from resonance. In general, the errors described herein are related to the variation in spacing of the conductors and are only indirectly a function of the variation in im. pedance. For a strip line, of course, the variation in spacing and impedance with distance are identical.

To conclude the discussion, consider the arbitrary condition that the error in incremental length must be kept below 10 per cent. Thus, from equation (284)

$$
0.10=[1 \cdots \cos (\phi / 2)]
$$

or

$$
\cos (\phi / 2)=0.90
$$

and

$$
\phi / 2=0.45 \text { radian } \quad .
$$

For the incremental length, $\Delta u$,

$$
\tan (\phi / 2)=\Delta y / 2 \Delta \mathrm{u}=0.48
$$

Finally,

$$
\mathrm{dy} / \mathrm{du}=0.963 .
$$

Hence, the section of tapered line whose taper, dy/du, reaches 0.963 has an error of 10 per cent per unit length. The total error, which is the summation of the errors for all the sections, depends upon the manner in which the line tapers. When a linear strip line is assumed, the spacing between conductors may be written

$$
\mathrm{y}=\mathrm{y}_{\mathrm{o}}[1+(\mathrm{u} / \mathrm{k})] .
$$


Then

$$
\mathrm{dy} / \mathrm{du}=\mathrm{y}_{\mathrm{o}} / \mathrm{k}
$$

From (295), for u equals $l$,

$$
\begin{aligned}
& y_{0} / \mathrm{k}=0.963 \\
& 1 / \mathrm{k}=0.963 / \mathrm{y}_{0}
\end{aligned}
$$

Thus, the taper constant of the linear strip line for which the error per unit length is 10 per cent is given by (299), where $1 / \mathrm{k}$ is the taper constant and $y_{o}$ is the initial spacing at $\underline{u}$ equals 0 . The total error in length is by equation (289)

$$
\begin{aligned}
E_{\ell} & =\int_{0}^{\ell}\left[1-1 / \sqrt{1+(1 / 4)\left(y_{0} / k\right)^{2}}\right] d u \\
& =\left[1-1 / \sqrt{1+(1 / 4)(0.963)^{2}}\right] \ell=0.10 l .
\end{aligned}
$$

Hence, the effective length of the line is $1.1 l$ instead of the measured length, $\ell$.

The results presented in the previous chapters are discussed and the limitations of these results are considered in this chapter.

The conclusions formulated as a result of this research are presented in the next chapter. 
CHAPTER VII

\section{CONC LUSIONS}

Introduction. - - The results discussed in the preceding chapter permit certain interesting conclusions pertaining to the resonant character istics of linear and exponential lines. A recapitulation of the most significant data, with appropriate conclusions, is given in this chapter. Resonant Properties. - - The information displayed in Fig. 34 and 40 represents the most important results which show the similarities and differences in the resonant properties of the three types of lines.

In general, the relative current and voltage standing waves (Fig. 34) are somewhat similar in shape on a normalized length scale. The most important difference in these characteristics is in the relative magnitudes of current required to obtain a given voltage at the open end. This property will be discussed further in the next section in connection with power dissipation.

The difference in standing waves $\left(\mathrm{I}_{\mathrm{N}}\right.$ and $\left.\mathrm{V}_{\mathrm{N}}\right)$ between the two tapered lines is small for values of $\mathrm{Z}_{\text {oS }} / \mathrm{Z}_{\text {oo }}$ less than 0.4 but becomes appreciable as this ratio increases. (For example, as $z_{o S} / z_{o o} a p-$ proaches 6.25 , the maximum value of the current for the exponential line becomes approximately 40 per cent greater than that for the linear line.)

The universal curves presented in Fig. 40 are concise represen tations of the quarter-wave resonance properties of the three types of (lossless) lines. The curves indicate that the ratio of length of the quarter-wave tapered line to that of the uniform line depends only upon the impedance ratio, $\mathrm{z}_{\mathrm{oS}} / \mathrm{Z}_{00}$. Alternatively, the curves show that the ratio of quarter-wave resonant frequency of the tapered line to that of the uniform line depends only upon the impedance ratio, $\mathrm{Z}_{\mathrm{OS}} / \mathrm{Z}_{\mathrm{OO}}$. 
In addition, the curves in Fig. 40 show that all negative lines are longer (or operate at a higher frequency) at quarter-wave resonance than the uniform line. Conversely, it is shown that all positive lines are shorter (or operate at a lower frequency) at quarter-wave resonance than the uniform line.

Except for the range of $Z_{\text {oo }} / Z_{\text {oS }}$ from 0.0 to 0.2 for the negative line and $Z_{o S} / Z_{\text {oo }}$ from 0.0 to 0.3 for the positive line, the curves in Fig. 40 for the two types of tapered lines are essentially identical. (Hence, for quarter-wave resonance over the range

$$
5.0 \geq \mathrm{z}_{\mathrm{oS}} / \mathrm{Z}_{\mathrm{OO}} \geq 0.3
$$

the simpler analytical expressions for the exponential line can be used, when desirable, for solutions of linear line problems.)

The above discussion permits the conclusions that are formulated and discussed below.

It is concluded that negative linear and exponential quarterwave lines can be used to replace comparable uniform lines for cases in which the physical length of the line is desired to be lengthened.

The significance of this property of negative lines is indicated by a problem of the type that follows. Consider the cyclotron problem in which the size of the dees, the dee-to-ground spacing, and the desired frequency combine to give a capacitive reactance of the dees sufficiently large so that the physical length of the uniform line (that would normally be used) is undesirably short. Then, in accordance with the above conclusion, a negative line can be used to increase the length of the dee stems. The nominal characteristic impedance of the tapered line will be equal to that of the uniform line at the dees and will decrease toward the opposite (shorted) end. The extent to which the dee stems will be lengthened will depend upon the achievable ratio of impedances $\mathrm{Z}_{\mathrm{OS}} / \mathrm{Z}_{\mathrm{OO}}$. 
It is concluded that negative linear and exponential quarterwave lines can be used to provide transmission lines of fixed length which will resonate at frequencies above the frequency determined by a uniform line of the same length.

To illustrate this property of tapered lines, consider the type of problem that follows. Currently, the ORNL 63-Inch Cyclotron operates at a frequency approximately one to five per cent below the nominal design value. Partly because of the physical limitations of the frequency adjustment mechanism, the desired operating frequency can not be obtained. Since the dee stems for this system consist of a pair of tubular conductors in a rectangular shield, direct modification of this uniform line to a negatively tapered linear or exponential line is not practical. However, in principle this can be done and, in accordance with the above conclusion, will result in an increase in operating frequency. (Since the results in Fig. 40 show that the frequency ratio depends only upon the ratio of impedances, $\mathrm{Z}_{\mathrm{OO}} / \mathrm{Z}_{\mathrm{OS}}$, it is appropriate to note that these results for the linear and exponential lines strongly suggest that a possible, and practical, solution to the above problem would be to decrease the spacing linearly between the $r-f$ shield and the two tubular conductors so that the nominal characteristic impedance varies in a logarithmic manner along the line. The resulting negative line would most surely increase the operating frequency.)

It is concluded that positive linear and exponential quarterwave lines can be used to replace comparable uniform lines for cases in which the physical length of the line is desired to be shortened.

This conclusion is the converse of the first and applies to problems in which the uniform line (that would normally be used) is undesirably long. As an illustration, the ORNL 63-Inch Cyclotron requires dee stems of greater length (16 feet) than is desired because the operating frequency is relatively low $(5 \mathrm{Mc} / \mathrm{sec})$. Thus, according to the above conclusion, the use of a positively tapered line would result in a de. crease in the length of the dee stems. The amount of this decrease in 
length would depend upon the value of $\mathrm{Z}_{\mathrm{oS}} / \mathrm{Z}_{\text {oo }}$ that can be obtained. It is concluded that positive linear and exponential quarter wave lines can be used to provide transmission lines of fixed length which will resonate at frequencies below the frequency determined by a uniform line of the same length.

This, of course, is the converse of the second conclusion and will not be discussed further.

Besides the conclusions given above, one additional conclusion pertaining to the resonant properties of the linear lines can be formu lated from the data in Fig. 14.

It is concluded that the novel resonant properties of linear lines are of interest for short lengths of line near quarterwave resonance.

That is, Fig. 14 shows that the quarter-wave resonant characteristics for the linear line approach those of the uniform line as the number of standing waves on the line increases.

The power considerations of quarter-wave lines are discussed in the next section.

Power Properties. - The most important results which compare the power dissipation characteristics of the three types of lines are presented in Fig. 41. These curves are derived on the basis that the voltages and nominal characteristic impedances at the open end are equal for all lines. In addition, all lines are operating at the same frequency and have equal (and constant) resistance per unit length.

An examination of the data in Fig. 41 shows that negative quarter-wave lines dissipate more power than the comparable uniform line. This results from two properties of the negative line. One, negative quarter-wave lines are longer than the uniform line as shown in Fig. 40. Two, the magnitude of the current distribution for the negative line is greater than for the uniform line (Fig. 34).

Conversely, the positive quarter-wave lines dissipate less power than the comparable uniform line because they are shorter and the magnitude of the current standing wave is smaller than for the uniform line. 
The power curves for the two positively tapered lines (Fig. 41) are essentially identical while, for the negatively tapered lines, the curves diverge for values of $\mathrm{Z}_{\mathrm{oo}} / \mathrm{Z}_{\text {oS }}$ below 0.7 . The principal reason for this divergence is due to the corresponding divergence of the magnitudes of the respective current standing waves (Fig. 34).

The above discussion permits the following conclusion:

It is concluded that positively tapered quarter-wave lines can be used advantageously in place of comparable uniform lines for cases in which the power loss is to be minimized.

An illustration of the significance of this property of positive lines is presented in Chapter VIII.

The tuning properties of the three lines are discussed in the next section.

Tuning Properties. --.In order to justify the conclusion which will be formulated below, it is first necessary to discuss Fig. 46 and 47 .

Consider several of the possible lines that can be derived from the strip line indicated in Fig. 46. As shown, the line is a positive linear line. If the spacing, $d_{s}$, at point $\mathrm{A}$ is held fixed and the line is pivoted about $\underline{\mathrm{A}}$ until the spacings at points $\underline{0}$ and $\underline{B}$ become equal to $d_{s}$, a uniform line results. Alternatively, if the spacing, $d_{0}$, is held fixed and the line is pivoted about $\underline{B}$ until $d_{s}$ becomes equal to $d_{0}$, another uniform line results. In the first case the uniform line has a low characteristic impedance determined by $d_{s}$, while in the second case it is much higher as determined by $d_{0}{ }^{\circ}$ Finally, if the spacing, $d_{m}$, is held fixed and the line pivoted about $\underline{0}$ until $d_{s}$ and $d_{0}$ exchange values, a negative linear line results. This last line is, of course, the same line as shown in Fig. 46 with the short at the opposite end. At the point in the process of pivoting the line about $\underline{0}$ in which the spacing at points $\underline{A}$ and $\underline{B}$ are equal to $\mathrm{d}_{\mathrm{m}}$, a third uniform line results. The characteristic impedance of this line is the average of those determined by $d_{s}$ and $d_{0}$. Fig. 47 shows the input reactance for the five lines just described. For clarity, numerical values are used. The length of each line is 16 feet and the characteristic impedances, determined by $\mathrm{d}_{\mathrm{s}}, \mathrm{d}_{\mathrm{m}}$, and $\mathrm{d}_{\mathrm{o}}$, are $15.7,68.4$, and $114.0 \mathrm{ohms}$, 
UNCLASSIFIED

ORNL-LR-DWG 18064

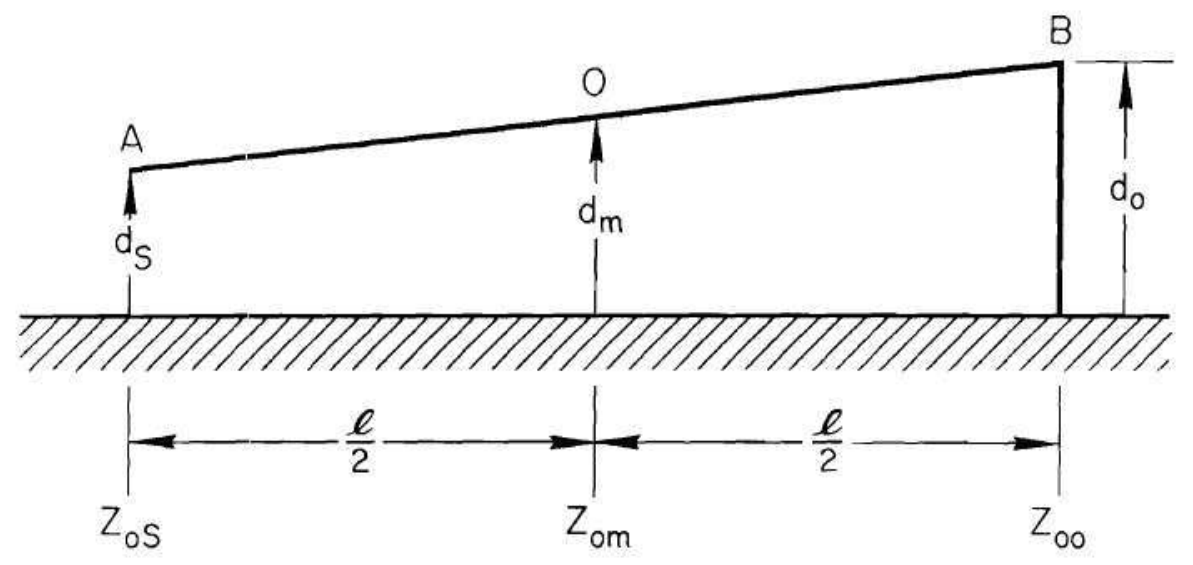

Fig. 46. Strip Line. 


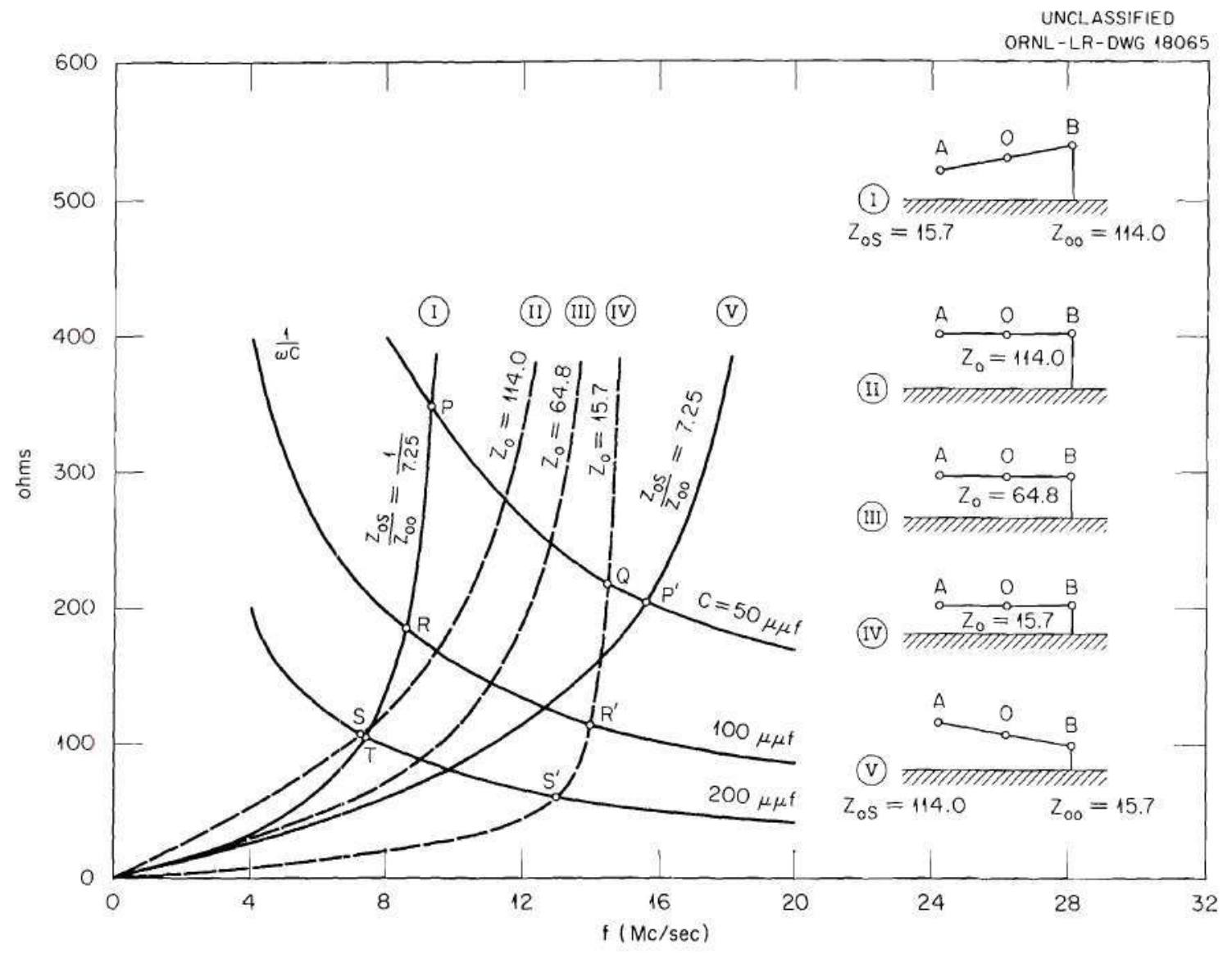

Fig. 47. Tuning Characteristics of Linear Lines vs Uniform Lines Where the Length Is Fixed and the Nominal Characteristic Impedance Is the Tuning Variable. 
respectively. Superimposed upon these five curves, without regard to sign, are capacitive reactance curves for four values of capacitance. The points of intersection of the two groups of curves correspond to resonances obtained by the assumption that the capacitance involved is terminating the line at the open end. Thus, if Line $I$ is pivoted about 0 while connected to 50 micro-microfarads of capacitance, the tuning range is determined by the points $\underline{P}$ and $\underline{P}$. If Line $I$ is pivoted about $\underline{A}$, the tuning range is determined by points $\underline{P}$ and $\underline{Q}$ and is approximately 82 per cent of PP'. If the terminal capacitance is 100 micromicrofarads, the tuning range $\underline{R} R^{\prime}$ of Line I pivoted about $\underline{A}$ is essentially the same as $\underline{P Q}$. Next, if the spacing of Line II is maintained uniform but gradually changed to that for Line IV, the tuning range is determined by the points $\underline{S}$ and $\underline{\mathbf{S}}$ ' if the terminal capacitance is 200 micro-microfarads. With this value of capacitance, Line I tunes over the same range, TS', when pivoted about $\underline{\text { A. }}$

The preceding discussion leads to the following conclusion:

It is concluded that linear and exponential lines add considerable flexibility to the solution of wide-range tuning problems in which the line is the variable parameter.

It is noted that the exponential line is included in the conclusion above although it is not discussed in Fig. 47. This is permissible when it is recalled that the exponential line has analogous characteristics similar to those (Fig. 47) of the linear line.

Summary. - - To accomplish the objectives of this investigation, analytical results to develop and extend the theory of the resonant properties of the tapered lines are presented in Chapters III and IV. These separate results for the exponential and linear lines are brought together to provide a common basis for comparison with similar properties of the uniform line in Chapter VI. Finally, the most significant results are summarized, with appropriate conclusions, in this chapter.

The next chapter presents two applications of the theory of the linear line. 
CHAPTER VIII

\section{APPLICATIONS}

Introduction.--This chapter presents two examples in which the properties of the linear line are applied to practical problems. The first example presents a solution to the cyclotron tuning problem which stimulated this research. The second example considers the results obtained by replacing a uniform line with a linear line in the solution of a wide range tuning problem.

Wide-Range Tuning of a Cyclotron Dee System.-.Consider the problem of designing a dee system which must handle hundreds of kilowatts of power and which must be adjusted to several specific frequencies from 6.7 to $11.8 \mathrm{Mc} / \mathrm{sec}$. For practical reasons, which are not discussed here, the given parameters of the system are as fol.lows:

1. The effective dee-to-dee capacitance is 300 micromic rofarads.

2. The desired length of the dee stems is approximately 16 feet.

3. The largest practical value of nominal characteristic impedance of the dee stems, at the dees, is $15.7 \mathrm{ohms}$.

It is shown in Chapter I (Fig. 5) that three possible tuning param. eters exist, namely, the length, $l$, the characteristic impedance, $z_{0}$. and the terminating capacitance, $C$. For purposes of comparison, all three of these possibilities are considered below.

Fig. 48 indicates the solution obtained by changing the length of a uniform line. As the length is changed from 32.2 to 16.0 feet, the system tunes from 6.7 to $11.8 \mathrm{Mc} / \mathrm{sec}$.

Fig. 49 shows the solution for which tuning is accomplished by adding capacitance at the dees. If 1550 micro-microfarads are added, the system covers the prescribed range. 


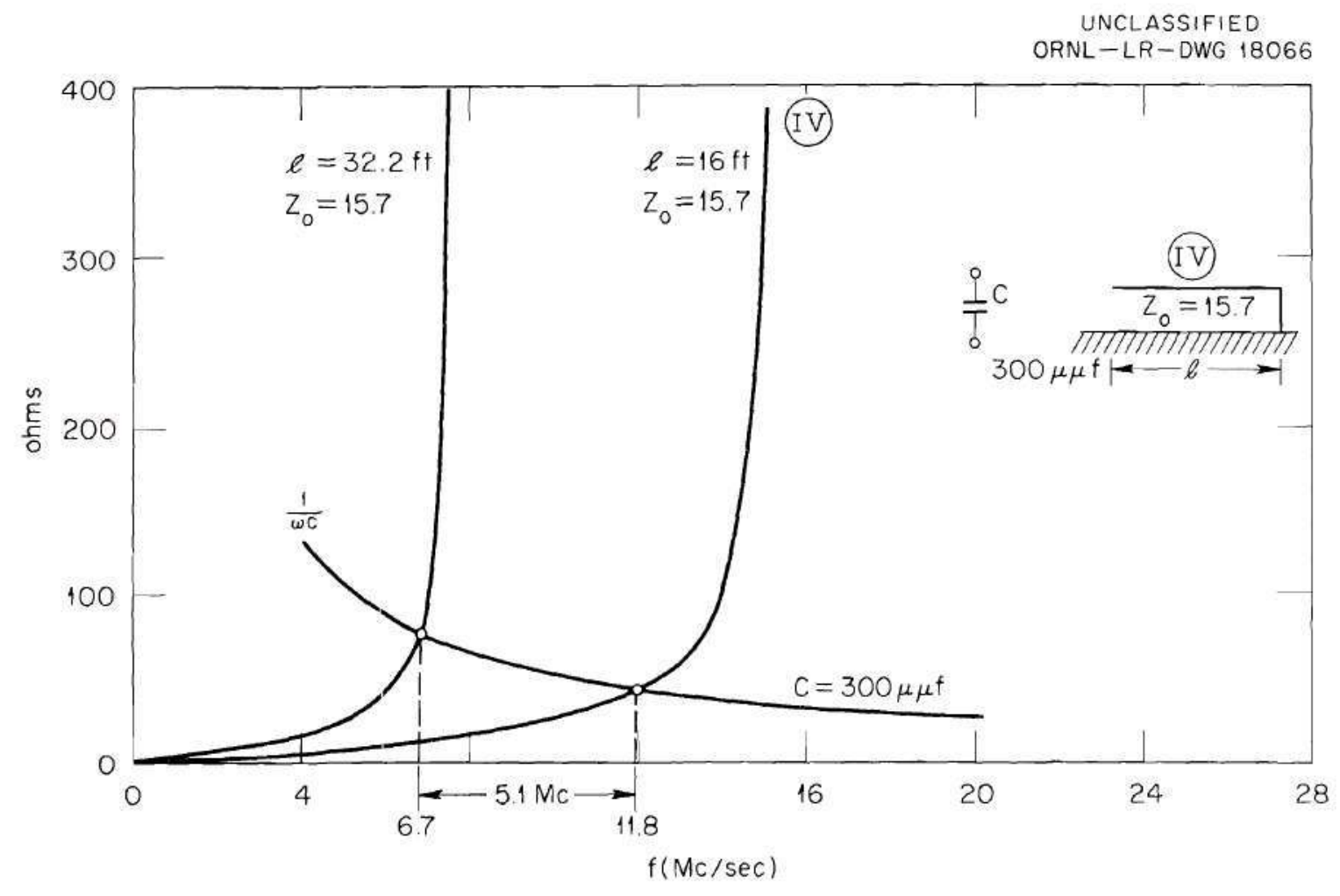

Fig. 48. Tuning $(6.7$ to $11.8 \mathrm{Mc} / \mathrm{sec})$ by Varying the Length of a Uniform Line. 
UNCLASSIFIED

ORNL-LR - DWG 18067

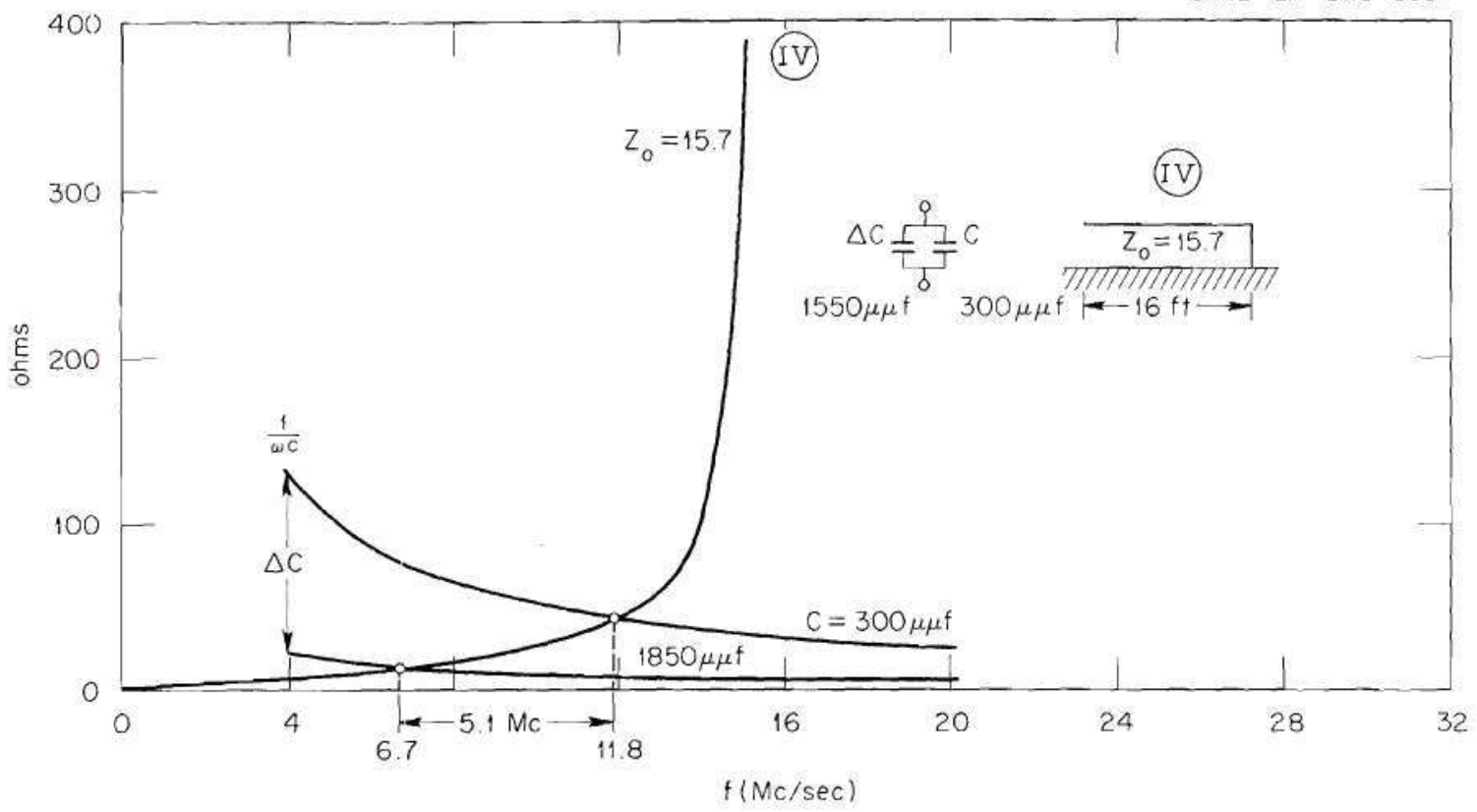

Fig. 49. Tuning $(6.7$ to $11.8 \mathrm{Mc} / \mathrm{sec})$ by Varying the Capacitance at the Open End of a Uniform Line. 
Finally, Fig. 50 presents a solution which employs a linear line. If a series of linear lines are obtained by pivoting Line I about point A such that the impedance at the shorted end assumes all values between 15.7 and $114.0 \mathrm{ohms}$, the desired tuning range is obtained.

With the assumption that the lines used in the three solutions are strip lines, the relative power dissipations can be estimated by the method that follows. From the above assumption, all of the lines have the same resistance per unit length since this quantity depends upon the width of the strip and not upon the spacing.

At the lowest frequency, $6.7 \mathrm{Mc} / \mathrm{sec}$, the electrical length of the line in the first solution is found to be 78.8 degrees. The current standing wave, from the shorted end, is the portion of a cosine wave between 0 and 78.7 degrees. This is expressed as follows:

$$
\begin{aligned}
& I(x)=I_{m} \cos \beta_{0} x \\
& \left(0 \leq \beta_{0} x \leq 78.8^{\circ}\right)
\end{aligned}
$$

The peak value, $I_{m}$, is established by the conditions at the dees. That is,

$$
I(l)=V_{D D} / X_{c}=I_{m} \cos \beta_{o} l
$$

where $\mathrm{V}_{\mathrm{DD}}$ is the dee-to-dee voltage and $\mathrm{X}_{\mathrm{C}}$ is the reactance of the dees. It follows from (301) and (302) that

$$
I(x)=\frac{V_{D D}}{x_{C} \cos \beta_{o} l} \cos \beta_{o} x
$$

For the case in question, $\beta_{\mathrm{o}} l$ is 78.8 degrees and $\mathrm{x}_{\mathrm{c}}$ from Fig. 48 is 78 ohms. Therefore,

$$
\begin{gathered}
I(x)=0.0626 V_{D D} \cos \beta_{\mathrm{O}} x \\
\left(0 \leq \beta_{\mathrm{O}} \mathrm{x} \leq 78.8^{\circ}\right)
\end{gathered}
$$




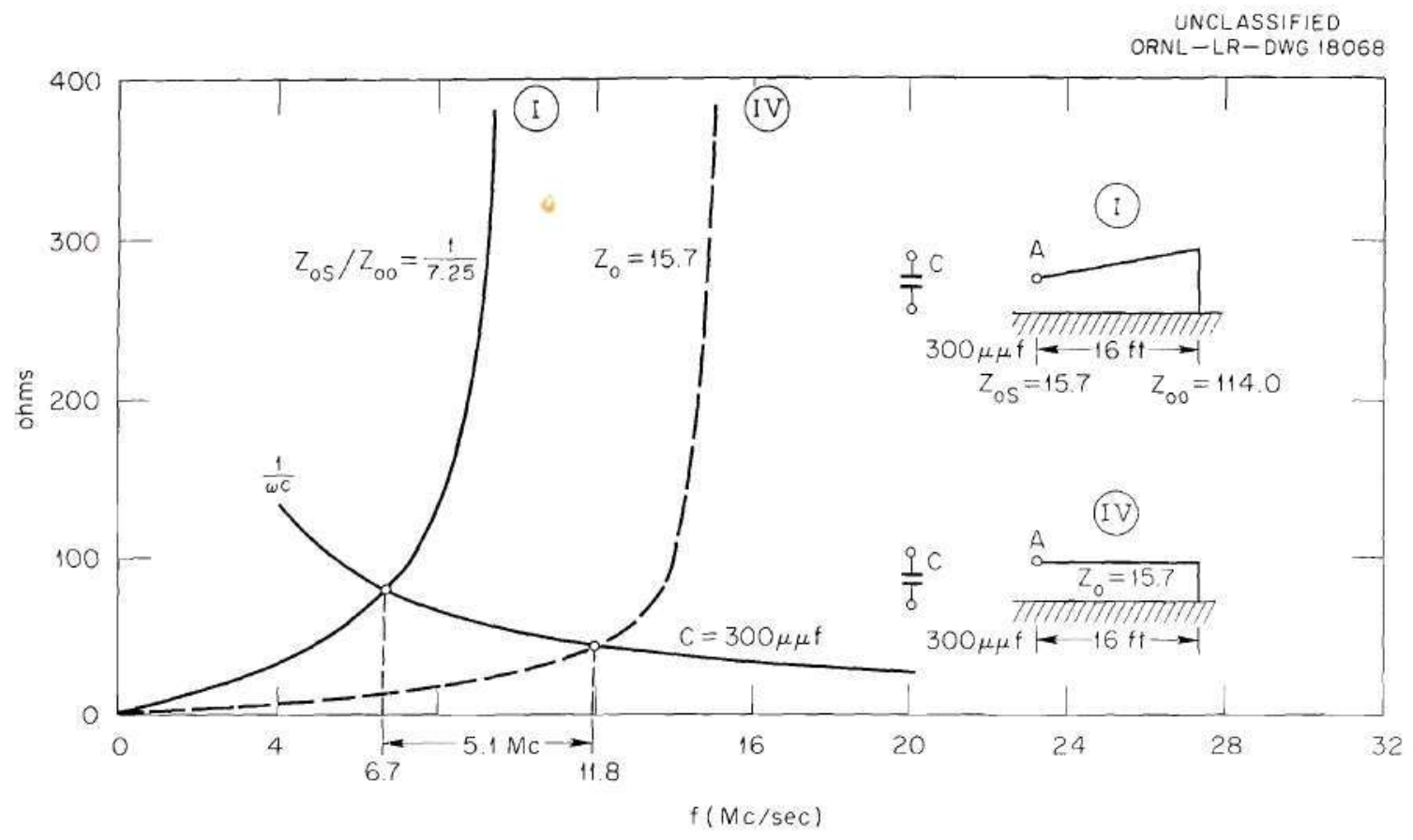

Fig. 50. Tuning $(6.7$ to $11.8 \mathrm{Mc} / \mathrm{sec})$ by Pivoting a Linear Strip Line about the Open End so that $Z_{o S} / Z_{o o}$ Ranges from $(15.7 / 114.0)$ to $(15.7 / 15.7)$. 
Similarly, for the second solution at $6.7 \mathrm{Mc} / \mathrm{sec}$,

$$
\begin{gathered}
I(x)=0.0810 V_{D D} \cos \beta_{O} x \\
\left(0 \leq \beta_{0} x \leq 39.2^{\circ}\right)
\end{gathered}
$$

where $X_{c}$ is 16 ohms. Finally, the equation for the current standing wave for the third solution is

$$
I(x)=\left(V_{D D} / Z_{o S}\right)\left[\frac{Y_{1 k} J_{0}-J_{1 k} Y_{0}}{I_{1 k} J_{1}\left(\rho_{0} \bar{u}\right)-J_{1 k} Y_{1}\left(\beta_{0} \bar{u}\right)}\right]
$$

from (259), where $l, \underline{k}, \bar{u}_{,}$and $z_{\text {oS }}$ are 16.00 feet, 18.56 feet, 2. 56 feet, and 15.7 ohms, respectively. For brevity (306) may be written as follows:

$$
I(x)=\left(V_{D D} / Z_{O S}\right) f(x)
$$

where $f(x)$ is the quantity in the brackets.

As shown in previous chapters the power dissipation is given by

$$
\mathrm{P}_{i}=(\mathrm{R} / 2) \int_{0}^{\ell_{i}}\left|\mathrm{I}_{\mathrm{i}}\right|^{2} \mathrm{~d} \ell_{i}
$$

For the three cases described above, the power expressions become

$$
\begin{aligned}
& P_{1}=(0.0626)^{2}\left(V_{D D} / 2\right)^{2} R \int_{0}^{32.2}\left(1+\cos 2 \beta_{0} x\right) d x, \\
& P_{2}=(0.0810)^{2}\left(V_{D D} / 2\right)^{2} R \int_{0}^{16.0}\left(1+\cos 2 \beta_{0} x\right) d x,
\end{aligned}
$$

and

$$
P_{3}=\left(V_{D D} / Z_{o S}\right)^{2}(R / 2) \int_{0}^{16.0} f^{2}(x) d x
$$


The first two integrals are easily obtained. The results are

$$
P_{1}=0.0359\left(V_{D D}\right)^{2} R
$$

and

$$
P_{2}=0.0452\left(V_{D D}\right)^{2} R
$$

Although the third integral is not as easily obtained, it can be approximated closely enough to permit comparison with (312) and (313). From Fig. 35 it is noted that $f(x)$, which is equivalent to $I_{N}(x / \ell)$, never exceeds the value $f(0)$. Hence, for purposes here, let $f(x)$ equal $f(0)$.

Then since

$$
\begin{aligned}
& f=6.7 \mathrm{Mc} / \mathrm{sec} \\
& \bar{u}=\mathrm{k}-\ell=2.56 \text { feet } \\
& \mathrm{x}=0 \\
& u=k-x=k=18.56 \text { feet, } \\
& \beta_{\mathrm{O}}=0.0428 \mathrm{radian} / \mathrm{foot}, \\
& \beta_{\mathrm{o}} \mathrm{u}=\beta_{\mathrm{o}} \mathrm{k}=0.795 \text { radian }, \\
& \beta_{\mathrm{o}} \overline{\mathrm{u}}=0.110 \mathrm{radian} \\
& Y_{1 k}=Y_{1}\left(\beta_{o} k\right)=-0.980, \\
& J_{0}=J_{0}\left(\beta_{0} k\right)=0.846 \\
& \mathrm{~J}_{1 \mathrm{k}}=\mathrm{J}_{1}\left(\beta_{\mathrm{o}} \mathrm{k}\right)=0.367 \quad, \\
& Y_{0}=Y_{0}\left(\beta_{0} k\right)=-0.087, \\
& \therefore \quad J_{1}=J_{1}\left(\beta_{0} \bar{u}\right)=0.055
\end{aligned}
$$


and

$$
f(0)=\frac{Y_{1 k} J_{0}-J_{1 k} Y_{0}}{Y_{1 k} J_{1}\left(\beta_{0} \bar{u}\right)-J_{1 k} Y_{1}\left(\beta_{0} \bar{u}\right)}=0.354 .
$$

If $(314)$ is substituted in ( 311$)$, the result is

$$
P_{3}=0.0041\left(V_{D D}\right)^{2} R
$$

It is noted that the actual power is less than that given by (315) since $f(x)$ is equal to or less than $f(0)$. The ratios of $P_{1}$ and $P_{2}$ to $P_{3}$ are as follows:

$$
P_{1} / P_{3}=8.6
$$

and

$$
\mathrm{P}_{2} / \mathrm{P}_{3}=10.8 .
$$

This shows that the linear line permits a considerable savings in power at $6.7 \mathrm{Mc} / \mathrm{sec}$. For frequencies between 6.7 and $11.8 \mathrm{Mc} / \mathrm{sec}$, the power ratios approach unity as the frequency approaches $11.8 \mathrm{Mc} / \mathrm{sec}$ since the lines are identical at the latter frequency.

A study of the first and second solutions shows other practical difficulties. The length required in the first solution is considered excessive from a practical viewpoint for two reasons. One, dee stems of this length require an unusually large vacuum tank. Two, such a length, when terminated by large dees, presents difficult mechanical problems even if the stems are suspended vertically to circumvent the cantilever problem. The large capacitance required by the second solution also presents difficult practical problems. The plates of such a capacitor must be spaced several inches apart to hold voltages of the order of hundreds of kilovolts. This factor, combined with the large 
capacitance required, leads to a capacitor of considerable size. It is shown in the literature ${ }^{5}$ that such a capacitor reduces the effective volt age amplification of the line and results in increased power dissipation. It is concluded that the use of a linear line, pivoted at the dees, offers the best solution to the problem. Wide-Range Tuning of a Tank Circuit. --As a second example, consider the problem of designing an amplifier tank circuit to cover the range from 120 to $300 \mathrm{Mc} / \mathrm{sec}$ with a fixed capacitance greater than 20 micromicrofarads at the tube. Meyerhoff and Graham ${ }^{23}$ present a solution which employs a section of uniform line and requires the capacitance values shown in Fig. 51. This circuit actually tunes from 100 to $300 \mathrm{Mc} / \mathrm{sec}$, a frequency ratio of 3:1. Rather than to present a formal solution to the problem, it is of more direct interest to consider the tuning range obtained by substituting a comparable linear line for the uniform line in Fig. 51. In this manner, the linear line may be compared with the uniform line for a particular case. That is to say。 unlike the first example in which the competition was among three different types of circuit components, this case offers competition between the linear and uniform lines - all other parameters are held constant. Consider the linear line parameters to be chosen arbitrarily as follows:

1. The length of the line is the same as that of the uniform line, 14 centimeters.

2. The nominal characteristic impedance at the midpoint of the line is equal to the characteristic impedance of the uniform line, $100 \mathrm{ohms}$.

3. The ratio of $Z_{\text {os }} / Z_{\text {oo }}$ is equal to 0.250 .

From the above values it follows that $\mathrm{Z}_{\mathrm{os}}$ and $\mathrm{Z}_{\mathrm{oo}}$ are 40 and $160 \mathrm{ohms}$, respectively.

Equation (187) in Chapter IV can be written as follows:

$$
Z_{L}=-(u / k)\left(\beta_{0} / y_{0}\right)\left[\frac{A_{o k} J_{1}-B_{o k} Y_{1}}{A_{o k} J_{0}-B_{o k} Y_{0}}\right]
$$




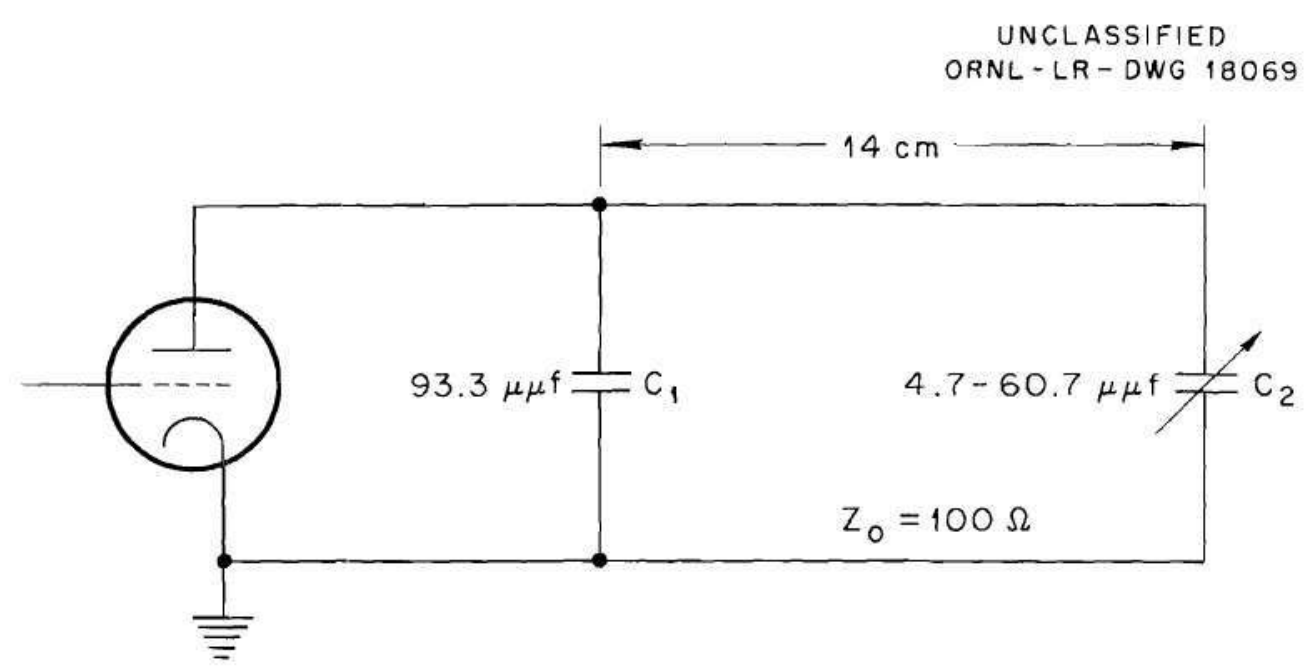

Fig. 51. Power Amplifier Tank Circuit which Tunes from 100 to $300 \mathrm{Mc} / \mathrm{sec}$. 
for a positive line, where

$$
\begin{aligned}
& A_{o k} \equiv Y_{o k}+\left(z_{o} / \beta_{o} Z_{R}\right) Y_{1 k}, \\
& B_{o k} \equiv J_{o k}+\left(z_{o} / \beta_{o} Z_{R}\right) J_{1 k},
\end{aligned}
$$

and

$$
z_{R}=-j X_{R}=-j\left(1 / \omega C_{2}\right)
$$

The conditions at the low end, $\omega_{1}$, and the high end, $\omega_{2}$, of the tuning range can be expressed as follows:

$$
1 / \omega_{1} C_{1}=\left|z_{L}\left(\omega_{1}, C_{2}{ }^{\prime}\right)\right|
$$

and

$$
1 / \omega_{2} C_{1}=\left|z_{L}\left(\omega_{2}, C_{2}^{\prime \prime}\right)\right|
$$

where $C_{2}^{\prime}$ and $C_{2}^{\prime \prime}$ are 60.7 and 4.67 micro-microfarads, respectively. If (319) and (320) are solved by approximate methods, the lower and upper frequencies are found to be 51 and $298 \mathrm{Mc} / \mathrm{sec}$. Thus, the tuning range has been increased from a ratio of $3: 1$ to $5.8: 1$, which is a substantial gain.

Although the above discussion does not consider the possible differences in line geometries and other factors which may present space or fabrication problems, it is presented for the purpose of demonstrating that, in principle, the application of short tapered lines is not limited to cyclotron problems. Line Geometries.--Since the preceding work has made repeated reference to the strip line geometry, it is appropriate to make a few remarks regarding practical line geometries. 
First, for most applications in which resonant transmission lines are used, the frequency is sufficiently high that shielding of the line is necessary. Second, since the concern is with tapered lines, the spacing between conductors must vary with length. This second condition presents practical fabrication problems, especially for the case in which the line sections are several feet in length. Shielded parallel wire lines utilizing conductors of small cross section are relatively easily fabricated into exponential and linear lines. This is not true, of course, for similar lines with conductors of large cross section. However, the strip line offers a comparatively simple geometry that can be used to approximate a shielded linear line quite easily. Fig. 52 shows the evolution of such a line. The linear line results from a linear change in the spacing, d, which is readily obtained with conductors of large or small cross section. Since no line geometry known to the author permits easy fabrication of exponential lines with conductors of large cross section, the emphasis is on linear lines. This, of course, reflects the author's interest in cyclotron applications since transmission lines of large cross section are inherent to the cyclotron problem.

Conclusions. - In addition to the general conclusions given in the preceding chapter, the following statements are made pertaining to the cyclotron problem that stimulated this research:

1. It is concluded that the linear line has important practical advantages for application to cyclotron power and tuning problems as illustrated in the first example above.

2. In particular from example one, it is concluded that the best solution to the tuning problem of a large variable frequency cyclotron is obtained by using a positive linear line for the dee stems.

The author's recommendations for further work in this field is discussed briefly in the next and final chapter. 

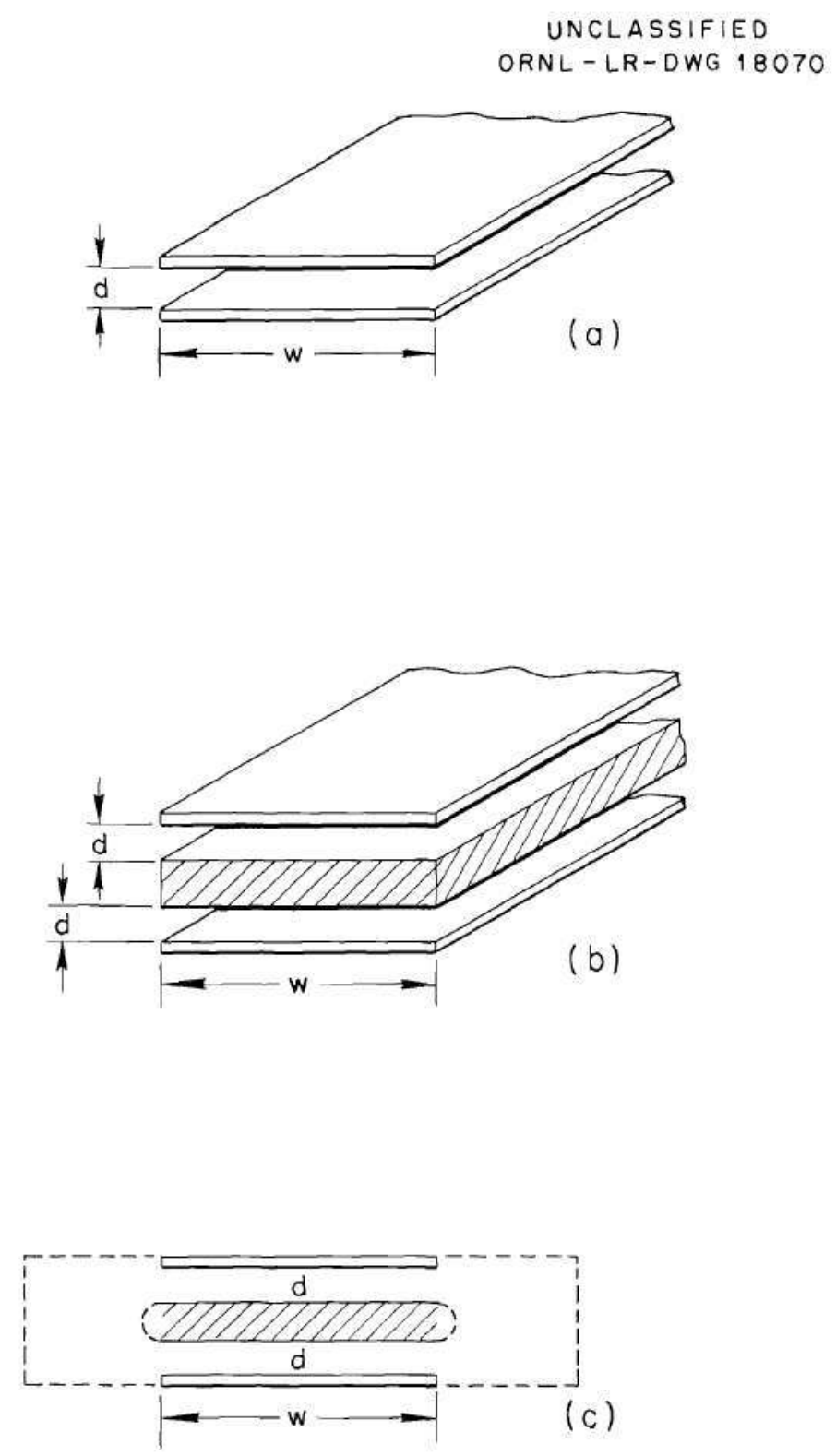

Fig. 52. (a) A Parallel Strip Line, (b) a Double Parallel Strip Line, and (c) a Shielded Coaxial Line Approximately Equivalent to a Strip Line. 


\section{CHAPTER IX}

\section{RECOMMENDATIONS}

Introduction, --.Some suggestions for further work on the resonant properties of nonuniform lines are presented in this chapter.

Suggested Extensions. - - An important area for further work of a nature similar to this research is with the type of nonuniform lines which can be obtained by a linear variation in the spacing between a cylindrical conductor and a ground plane. Since the characteristic impedance of such a geometry is a logarithmic function of the spacing, the general equations for the line are not easily solved analytically. However, with modern computing methods, the labor should not be prohibitive.

The results of such a study should prove quite useful for design and analysis purposes. Since many uniform lines of this form are in existence, the results should be important for interpreting the effects of inhomogeneities in the uniform line. In addition, since nonuniform lines of this geometry are easily fabricated, it appears likely that they may find wide application for problems in which only a modest "taper" is required. Due to the logarithmic variation of the impedance with spacing, this type of line is expected to present the greatest interest in the region for which $\mathrm{Z}_{\text {os }} / \mathrm{Z}_{\text {oo }}$ is near unity.

As an illustration, the results of the study suggested could be applied to the case of an existing quarter-wave uniform line resonator consisting of a pair of tubular conductors in a rectangular shield. It has been the author's experience that it frequently becomes desirable to raise or lower the resonant frequency range by a modest amount. This can be done by changing the spacing of the tubular conductors at one end relative to that at the other. In most cases, it is not possible to obtain a large value for $\mathrm{z}_{\text {os }} / \mathrm{Z}_{\text {oo }}$ (or $\mathrm{z}_{\text {oo }} / \mathrm{Z}_{\text {os }}$ ). 
A second and more ambitious study related to this work would be a comprehensive investigation of a considerable number of different types of nonuniform lines with the object of determining the general trends of the resonant properties as a function of the different types of lines.

The general method suggested for conducting either of the studies indicated is outlined briefly in the next section.

Suggested Method of Attack. --Consider the two basic transmission line equations. They are

$$
d V / d x=-z(x) I(x)
$$

and

$$
\mathrm{dI} / \mathrm{dx}=-\mathrm{y}(\mathrm{x}) \mathrm{V}(\mathrm{x})
$$

where

$$
z(x)=z_{a}+\bar{z}(x)
$$

and

$$
y(x) \equiv y_{a}+\bar{y}(x)
$$

$\mathrm{z}_{\mathrm{a}}$ and $\mathrm{y}_{\mathrm{a}}$ in (323) and (324) may be regarded as the average value of $z(x)$ and $y(x)$, respectively. That is

$$
z_{a} \equiv(1 / \ell) \int_{0}^{\ell} z(x) d x,
$$

and

$$
y_{a} \equiv(1 / \ell) \int_{0}^{l} y(x) d x
$$

Then

$$
d V / d x=-z_{a} I(x)-\bar{z}(x) I(x)
$$


and

$$
d I / d x=-y_{a} V(x)-\bar{y}(x) V(x)
$$

are shown by Schelkun off ${ }^{10}$ to have solutions of the form,

$$
\begin{aligned}
V(x)=V_{0}(x) & -\int_{0}^{x} \bar{z}(a) I(a) \cosh \gamma_{a}(x-a) d a \\
& +Z_{a} \int_{0}^{x} y(a) V(a) \sinh \gamma_{a}(x-a) d a
\end{aligned}
$$

and

$$
\begin{gathered}
I(x)=I_{0}(x)+\left(1 / Z_{a}\right) \int_{0}^{x} \bar{z}(a) I(a) \sinh \gamma_{a}(x-a) d a \\
-\int_{0}^{x} \bar{y}(a) V(a) \cosh \gamma_{a}(x-a) d a
\end{gathered}
$$

where

$$
\begin{aligned}
& V_{0}(x)=V_{0} \cosh \gamma_{a} x-z_{a} I_{0} \sinh \gamma_{a} x, \\
& I_{0}(x)=I_{0} \cosh \gamma_{a} x-\left(V_{0} / z_{a}\right) \sinh \gamma_{a} x, \\
& Z_{a}=\sqrt{z_{a} / y_{a}}, \\
& \gamma_{a}=\sqrt{z_{a} y_{a}}, \\
& V_{0}=V(0),
\end{aligned}
$$

and

$$
I_{0}=I(0)
$$


The above integrals may be calculated by the method of successive approximations. Thus,

$$
V(x)=\sum_{i=0}^{n} V_{i}(x)
$$

and

$$
I(x)=\sum_{i=0}^{n} I_{i}(x),
$$

where

$$
\begin{aligned}
V_{i+1}(x)= & -\int_{0}^{x} \bar{z}(a) I_{i}(a) \cosh \gamma_{a}(x-a) d a \\
& +z_{a} \int_{0}^{x} \bar{y}(a) V_{i}(a) \sinh \gamma_{a}(x-a) d a
\end{aligned}
$$

and

$$
\begin{aligned}
I_{i+1}(x)=\left(1 / z_{a}\right) & \int_{0}^{x} \bar{z}(a) I_{i}(a) \sinh \gamma_{a}(x-a) d a \\
& -\int_{0}^{x} \bar{y}(a) V_{i}(a) \cosh \gamma_{a}(x-a) d a .
\end{aligned}
$$

For a more comprehensive discussion of this method, the reader is referred to Carson's ${ }^{8}$ and Schelkunoff's ${ }^{1.0}$ works.

Concluding Remarks. ---In conclusion, it is hoped that the results of this research will find application to a variety of resonance problems and will stimulate further work in this field. 
A P P ENDIX 


\section{THE PARALLEL-STRIP LINE}

The uniform strip line consists of two flat conductors of width, whose adjacent parallel surfaces are spaced a distance, $\underline{\text { d, }}$ apart as shown in Fig. 52. For d very much less than w, the system propagates a TEM wave analogous to the principal wave propagated between parallel planes of semi-infinite extent. The specified condition $(d<<w)$ is equivalent to neglecting fringe effects of the electro magnetic field. The field plot at any cross section transverse to the direction of propagation has been shown ${ }^{*}$ to be a rectangular grid. That is, the gradient of the electric field is a constant equal in magnitude to the voltage divided by the distance between conductors. The current is uniformly distributed across the conductors such that the $r-f$ resistance ${ }^{* * *}$ per unit length of line is the skin effect resistance divided by $w_{0}$ Specifically, the resistance is independent of the spacing between conductors. Hence, if this uniform strip line is modified slightly by inclining the planes of the two conductors with respect to each other, a linear line results. Then to the first approximation, the field plot at a typical cross section has not changed significantly and the resistance per unit length is approximately the same as before. Thus, the assumption that the resistance per unit length of a linear line is constant is easily obtained with a practical line.

*E. C. Jordan, Electromagnetic Waves and Radiating Systems, New York: Prentice-Hall, Inc., 1950, p. 188.

*** Simon Ramo and John R. Whinnery, Fields and Waves in Modern Radio, New York: John Wiley and Sons, Inc., 1944, Chap. 9. 
II

\section{LIST OF EQUATIONS FOR THE UNIFORM, EXPONENTIAL AND LINEAR LINES}

The voltage and current equations for the three types of lines are listed to facilitate comparison.

\section{General Equations}

Uniform Line: $\quad V=V_{R}\left[\cosh \gamma x+\left(Z_{o} / Z_{R}\right) \sinh \gamma x\right]$

Exponential Line: $\quad V_{E}=V_{R}\left[\cosh \Gamma x+\left\{(\delta / T)+\left(z_{o} / \Gamma Z_{R}\right)\right\} \sinh \Gamma x\right] e^{-\delta x}$ Linear Line: $\quad V_{L}=V_{R}(\pi G u / 2)\left\{\left[Y_{0}(G k)+\left(z_{0} / G Z_{R}\right) Y_{1}(G k)\right] J_{1}(G u)\right.$ $\left.-\left[J_{0}(G k)+\left(z_{0} / G Z_{R}\right) J_{1}(G k)\right] \quad Y_{1}(G u)\right\}$

Uniform Line: $\quad I=I_{R}\left[\cosh \gamma x+\left(Z_{R} / Z_{0}\right) \sinh \gamma x\right]$ Exponential Line: $I_{E}=I_{R}\left[\cosh T x-\left\{(\delta / T)-\left(y_{0} Z_{R} / T\right)\right\} \sinh \Gamma x\right] e^{\delta x}$ Linear Line: $\quad I_{L}= \pm I_{R}\left(\pi y_{0} k / 2\right)\left\{\left[Z_{R} Y_{0}(G k) \pm\left(z_{0} / G\right) Y_{1}(G k)\right] J_{0}(G u)\right.$ $\left.-\left[\mathrm{Z}_{\mathrm{R}} \mathrm{J}_{\mathrm{o}}(\mathrm{Gk}) \pm\left(\mathrm{z}_{\mathrm{o}} / \mathrm{G}\right) \mathrm{J}_{1}(\mathrm{Gk})\right] \mathrm{Y}_{\mathrm{o}}(\mathrm{Gu})\right\}$

\section{Lossless Line Equations}

Uniform Line: $\quad V=V_{R}\left[\cos \beta_{0} x+j\left(Z_{0} / Z_{R}\right) \sin \beta_{0} x\right]$

Exponential Line: $V_{E:}=V_{R}\left[\cos \beta x+\left\{(\delta / \beta)+\left(z_{0} / \beta Z_{R}\right)\right\} \sin \beta x\right] e^{-\delta x}$ Linear Line: $\quad V_{L_{1}}=V_{R}\left(\pi \beta_{o} u / 2\right)\left\{\left[Y_{o k} \pm\left(z_{o} / \beta_{o} Z_{R}\right) Y_{1 k}\right] J_{J}\left(\beta_{o} u\right)\right.$ $\left.-\left[J_{o k} \pm\left(z_{o} / \beta_{o} Z_{R}\right) J_{1 k}\right] Y_{1}\left(\beta_{o} u\right)\right\}$ 
Uniform Line: $\quad I=I_{R}\left[\cos \beta_{0} x+j\left(Z_{R} / Z_{0}\right) \sin \beta_{o} x\right]$

Exponential Line: $I_{E}=I_{R}\left[\cos \beta x-\left\{(\delta / \beta)-\left(y_{0} / \beta Z_{R}\right)\right) \sin \beta x\right] e^{\delta x}$

Linear Line: $\quad I_{L}= \pm I_{R}\left(\pi y_{0} k / 2\right)\left\{\left[Z_{R} Y_{o k} \pm\left(z_{o} / \beta_{o}\right) Y_{1 k}\right] J_{0}\left(\beta_{o} u\right)\right.$

$$
\left.-\left[\mathrm{Z}_{\mathrm{R}} \mathrm{J}_{\mathrm{ok}} \pm\left(\mathrm{z}_{\mathrm{o}} / \beta_{\mathrm{o}}\right) \mathrm{J}_{1 \mathrm{k}}\right] \mathrm{Y}_{\mathrm{o}}\left(\beta_{\mathrm{o}} \mathrm{u}\right)\right\}
$$

\section{Lossless Shorted Line Equations}

Uniform Line: $\quad V=j V_{S} \sin \beta_{\mathrm{O}} \mathrm{x}$

Exponential Line: $v_{E}=v_{E S}\left[\left(e^{\delta l} / \sin \beta l\right) \sin \beta x\right] \quad e^{-\delta x}$

Linear Line: $\quad V_{L}=V_{L S}\left(Z_{o L} / Z_{o S}\right)\left[\frac{Y_{1 k} J_{1}\left(\beta_{o} u\right)-J_{1 k} Y_{1}\left(\beta_{o} u\right)}{Y_{1 k} J_{1}\left(\beta_{0} \bar{u}\right)-J_{1 k} Y_{1}\left(\beta_{o} \bar{u}\right)}\right]$

Uniform Line: $\quad I=I_{R} \cos \beta_{O} x$

Exponential Line: $I_{E}=I_{R}[\cos \beta x-(\delta / \beta) \sin \beta x] e^{\delta x}$

Linear Line: $\quad I_{L}=I_{R}\left(\pi \beta_{0} k / 2\right)\left[Y_{1 k} J_{o}\left(\beta_{o} u\right)-J_{l k} Y_{o}\left(\beta_{0} u\right)\right]$ 


\section{DEVELOPMENT OF EQUATION (274)}

Consider the propagation of a radial wave guided by the two semi-infinite conducting planes shown in Fig. 44. For propagation of such a wave in a dielectric medium, there is no variation in the field components in the $\underline{x}$ direction,

$$
E_{r}=E_{x}=H_{x}=H_{\varnothing}=\sigma=0 \text {, }
$$

where $E_{r}, E_{x}, H_{r}, H_{\phi}$ are the maximum values of the field components in the direction indicated by the respective subscripts and $\sigma$ is the conductivity of the propagating medium. Then, if the electric and mag w netic field intensities are harmonic functions of time,

$$
\begin{aligned}
& \underset{\sim}{E}=\underset{\sim}{a} E_{\phi} e^{j \omega t} \\
& \underset{\sim}{H}=\underset{\sim}{\underset{x}{a}} H_{x} e^{j \omega t},
\end{aligned}
$$

it can be shown by direct application of the two (Maxwell's) equations **

$$
\begin{aligned}
& \operatorname{curl} \underset{\sim}{\mathrm{E}}=\underset{\sim}{\mathrm{i}}+\partial \underset{\sim}{\mathrm{D}} / \partial \mathrm{t} \\
& \operatorname{curl} \underset{\sim}{\mathrm{H}}=-\partial \underset{\sim}{\mathrm{B}} / \partial \mathrm{t}
\end{aligned}
$$

that the following wave equation results

$$
d^{2} E / d r^{2}+(1 / r) d E_{\phi} / d r-\left[\left(1 / r^{2}\right)-\mu \in \omega^{2}\right] E_{\phi}=0
$$

* See for example, Hyper and Ultrahigh Frequency Engineering, R. I. Sarbacher and W. A. Edson, John Wiley and Sons, Inc, , p. 231-232. 
where $\mu$ and $\epsilon$ are the magnetic inductive capacity and the electric inductive capacity of the propagating medium. The quantity $\omega$ is the angular frequency of the field components. Equation (346) is recognized as Bessel's equation in cylindrical coordinates and has a solution of the form

$$
E_{\phi}=C_{1} J_{1}\left(\beta_{0} r\right)+C_{2} Y_{1}\left(\beta_{0} r\right)
$$

where $C_{1}$ and $C_{2}$ are constants of integration and, $J_{1}\left(\beta_{0} r\right)$ and $\mathrm{Y}_{1}\left(\beta_{\mathrm{O}} \mathrm{r}\right)$ are Bessel functions of the first and second kind of order one. Also,

$$
\beta_{0}=\omega \sqrt{\mu \epsilon}
$$

Similarly, it can be shown that

$$
H_{x}=j \sqrt{\mu / \epsilon}\left[C_{1} J_{0}\left(\beta_{0} r\right)+C_{2} Y_{0}\left(\beta_{0} r\right)\right]
$$

where $J_{0}\left(\beta_{0} r\right)$ and $Y_{0}\left(\beta_{0} r\right)$ are Bessel functions of the first and second kind of order zero.

From electromagnetic field theory, the current density at a position $\underline{r}$ at the inner surface of the conducting plane, $\varnothing=0$, is

$$
\underset{\sim}{\mathrm{J}}=\underset{\sim}{\mathrm{n}} \mathrm{X} \underset{\sim}{\mathrm{H}}
$$

from which

$$
\mathrm{J}_{\mathbf{r}}=-\mathrm{H}_{\mathbf{x}}
$$

The total current at the surface, in a strip

$$
x_{2}-x_{1}=w
$$


units wide, is

$$
\begin{aligned}
I & =J_{r} w=-w H_{x} \\
& =-j w \sqrt{\epsilon / \mu}\left[C_{1} J_{0}\left(\beta_{0} r\right)+C_{2} Y_{0}\left(\beta_{0} r\right)\right]
\end{aligned}
$$

The voltage between the two planes,

$$
\begin{aligned}
& \phi=0 \\
& \phi=\varnothing \quad
\end{aligned}
$$

at a distance $\underline{r}$ from the origin, is

$$
V=-\int_{0}^{s} E_{\phi} d s
$$

where

$$
\mathrm{s}=\varnothing \mathrm{r} .
$$

Since $E_{\phi}$ is not a function of $\varnothing$,

$$
\begin{aligned}
V & =-E_{\phi^{S}}=-\phi r E_{\phi} \\
& =-\phi r\left[C_{1} J_{1}\left(\beta_{0} r\right)+C_{2} Y_{1}\left(\beta_{0} r\right)\right] .
\end{aligned}
$$

By using the following boundary conditions in (353) and (354)

$$
\begin{aligned}
& V\left(r_{1}\right)=0 \\
& I\left(r_{1}\right)=I_{R}
\end{aligned}
$$


and, by using the following equality from the theory of Bessel functions

$$
J_{0}\left(\beta_{0} r_{1}\right) Y_{1}\left(\beta_{0} r_{1}\right)-J_{1}\left(\beta_{0} r_{1}\right) Y_{0}\left(\beta_{0} r_{1}\right)=-2 / \pi \beta_{0} r_{1},
$$

the expressions for voltage and current become

$$
\begin{aligned}
& V=j\left(\pi \mu \omega r_{1} r / 2 w\right)\left[Y_{1}\left(\beta_{0} r_{1}\right) J_{1}\left(\beta_{0} r\right)-J_{1}\left(\beta_{0} r_{1}\right) Y_{1}\left(\beta_{0} r\right)\right] \\
& I=-\left(\pi \beta_{0} r_{1} / 2\right)\left[Y_{1}\left(\beta_{0} r_{1}\right) J_{0}\left(\beta_{0} r\right)-J_{1}\left(\beta_{0} r_{1}\right) Y_{0}\left(\beta_{0} r\right)\right] .
\end{aligned}
$$

The ratio of $(360)$ and $(361)$ is

$$
\mathrm{Z}=\mathrm{V} / \mathrm{I}
$$

which is seen to be of the form

$$
Z=-j Z_{o r}\left[\frac{Y_{1}\left(\beta_{0} r_{1}\right) J_{1}\left(\beta_{0} r\right)-J_{1}\left(\beta_{0} r_{1}\right) Y_{1}\left(\beta_{0} r\right)}{Y_{1}\left(\beta_{0} r_{1}\right) J_{0}\left(\beta_{0} r\right)-J_{1}\left(\beta_{0} r_{1}\right) Y_{0}\left(\beta_{0} r\right)}\right]
$$

where

$$
z_{\text {or }}=\sqrt{\mu / \epsilon}\left(\phi_{r} / w\right)
$$

For an air dielectric,

$$
\sqrt{\mu / \epsilon}=377
$$

and

$$
\mathrm{z}_{\text {or }}=377 \phi \mathrm{r} / \mathrm{w}
$$


Equation (363) is seen to be identical in form to equation (246) in Chapter IV. Thus, from (363), $\mathrm{Z}_{\text {or }}$ corresponds to $\mathrm{Z}_{\mathrm{OL}}$ in (264). Hence, the conducting planes (of width w) in the above field theory analysis are one form of a linear line. 
B IB LIOGRA P H Y 
1. Marton, L., Advances in Electronics, New York: Academic Press Inc., 1948, Vol. I, 269-316.

2. Electronuclear Research Division, Proposal for a MultipleParticle Heavy-Ion Cyclotron, A Report, Oak Ridge National Laboratory, Oak Ridge, Tenn., (ORNL CF-53-10-63).

3. Fulbright, H. W., D. A. Bromley, J. A. Bruner, R. Hawrylak, and A. Hamann, Preliminary Report on the New 8-Mev Variable Energy Cyclotron at the University of Rochester, Physics Department, Rochester, New York, (NYO-6541), Nov, 12, 1954.

4. Smith, Bob H., A 90-Inch Cyclotron with an Adjustable-Energy External Beam, A Report, University of California Radiation Laboratory, Berkeley, California, (UCRL-2620), June 9, 1954.

5. Donaldson, M. R., R. E. Worsham, and N. F. Ziegler, "A Study of a Variable Frequency Cyclotron Resonant System, " 1955 I. R. E. Convention Record, Part 10, 191-198, March (1955).

6. Heaviside, Oliver, Electromagnetic Theory, Unabridged Edition of Vol. I, II, and III, New York: Dover Publications, Inc., 1950.

7. Ravut, C., "Propagation des Courants Sinusoidaux Sur des Lignes Quelconques," Revue Generale de l'Electricite, Vol. 7 , Nol. 19, 611-615, May (1920).

8. Carson, J. R., "Propagation of Periodic Currents over Non uniform Lines," Electrician, Vol. 86, No. 10, 272-273, March 4 (1921).

9. Thomas, F., "Propagation du Courant Sur les Lignes Quelconques," Revue Generale de 1'Electricite, Vol. 26, No. 15, 567-569, Oct. (1929).

10. Schelkunoff, S. A., Electromagnetic Waves, New York: D. Van Nostrand, 1943, Chap. 7.

11. Ballantine, Stuart, "Nonuniform Lumped Electrical Lines: I. The Conical Lines," Journal of Franklin Institute, Vol. 203, 561 582, April (1927).

12. Arnold, J. W., and P. F. Bechberger, "Sinusoidal Currents in Linearly Tapered Loaded Transmission Lines," Proc。I。R. E。, Vol. 19, No. 2, 304-310, Feb. (1931).

13. Frederici, M. , "Su di un auovo tipo di cavo electtrico disunforme," Rendiconti della R. Accad. Naz, die Linzei, Vol. XIII, 6a, 128-132, gennaio 1931 . 
14. Arnold, J. W., and R. C. Taylor, "Linearly Tapered Loaded Transmission Lines," Proc. I. R. E., Vol. 20, No. 11, $1811-1817$, Nov, (1932).

15. Starr, A. T., "The Nonuniform Transmission Line," Proc. I. R. E., Vol. 20, No. 6, 1052-1063, June (1932).

16. Burrows, C. R., "The Exponential Transmission Line," B. S. T. J., Vol. 17, 555-573, (1938).

17. Walker, L. R., and N. Wax, "Nonuniform Transmission Lines and Reflection Coefficients, "Journal of Applied Physics, Vol. 17, 1043-1045, Dec. (1946).

18. Selgin, P. J., Electrical Transmission in Steady State, New York: McGraw-Hill, 1946, Chap. XIII.

19. Wheeler, H. A., "Transmission Lines with Exponential Taper," Proc. I. R. E., Vo1. 27, 65-71, Jan. (1939).

20. Schatz, E. R., and E. M. Williams, "Pulse Transients in Exponential Transmission Lines," Proc. I. R. E., Vol. 38 , 1208-1212, Oct. (1950).

21. Ramo, Simo, and John R. Whinnery, Fields and Waves in Modern Radio, New York: John Wiley and Sons, Inc., 1944, Chap. 8.

22. Jordan, Edward C., Electromagnetic Waves and Radiating Systems, New York: Prentíce-Hall, Inc., 1950, Chap. 8.

23. Myerhoff, Alan A., and Robert Graham, Jr., "Fixed-Length Transmission Lines as Circuit Elements," Proc. I. R. E., Vol. 41, 267, Feb. (1953). 


\section{VITA}

Merle Richard Donaldson was born in Silverdale, Kansas, to Harry R. and Alma F. Donaldson on April 7, 1920. He was married in 1943 to Miss Maideth Erlaine Bennett of Asheville, North Carolina, and has two children.

He attended public schools in Hazelton, Kansas, and Grainola, Oklahoma, where he graduated from Grainola High School in 1938.

During 1938 and 1939 he was employed by the U. S. Department of Agriculture at Valley Falls, Kansas. In 1940, he joined the U. S. Navy and served for a period of six years.

He entered Carson-Newman College at Jefferson City, Tennessee, in 1943 in the Navy V -12 program and transferred to Georgia Institute of Technology in 1944 where he received the B. E. E. degree in 1946 and the M. S. E. E. degree in 1947. From 1947 to 1950 he was a special graduate student at Georgia Tech. During 1951 and 1952 he attended the University of Tennessee at Knoxville, Tennessee, as a special graduate student.

He joined the faculty of the School of Electrical Engineering at Georgia Tech in the spring of 1946 as a part-time Instructor in Electrical Engineering and became an Instructor in 1947 and an Assistant Professor in 1950. For the fall quarter of 1950, he accepted an appointment as a Research Participant at the Oak Ridge National Laboratory, Oak Ridge, Tennessee. During this period he received orders to active duty with the U. S. Navy. These orders were subsequently cancelled, at the request of the Atomic Energy Commission, to permit him to continue work on a cyclotron project. He joined the Oak Ridge National Laboratory as a permanent employee of the Electronuclear Research Division in January 1951 and has worked the re since that date. During this period he worked on a succession of cyclotron projects which include the ORNL 63-, 44-。 and 48-inch machines. In 1953 he became a Project Leader in charge of $r-f$ design and development and was promoted to Senior Engineer in 1956. 